\title{
Performance Enhancement of High Order LDPC Coded Modulation Systems With Application in Mobile Backhaul Networks
}

\author{
by \\ Peyman Neshaastegaran, M.Sc. \\ A dissertation submitted to the \\ Faculty of Graduate and Postdoctoral Affairs \\ in partial fulfillment of the requirements for the degree of
}

Doctor of Philosophy in Electrical Engineering

Ottawa-Carleton Institute for Electrical and Computer Engineering

Department of Systems and Computer Engineering

Carleton University

Ottawa, Ontario

February, 2021

(C) Copyright

Peyman Neshaastegaran, 2021 


\section{Abstract}

Wireless backhaul communication systems play a crucial role in the infrastructure of current cellular networks. In light of the expensive costs of fiber deployment, the importance of these links is anticipated to continue over the next few decades. Point-to-point microwave radio links (PtPMRLs) are the backbone of every wireless backhaul system. To achieve higher spectral efficiencies, the PtPMRL are designed based on the concurrent exploitation of high-order modulations and powerful forward error correcting codes. An example of such design paradigm is the high-order lowdensity-parity-check coded modulation systems (LDPC-CMSs). In this thesis two key challenges in the high-order LDPC-CMSs are overcome.

In our first contribution, we consider the problem of oscillator phase hit $(\mathrm{PH})$ in microwave backhaul communication links. PH results in a temporary link loss in the communication system and bears an expensive cost on the operators. In this work, we propose a two-stage solution to mitigate PH. In the first stage, we use NeymanPearson binary hypothesis testing to develop a low cost $\mathrm{PH}$ detection algorithm. In this test, a likelihood ratio test is designed and the optimal detection threshold is analytically calculated. The proposed PH detection scheme entails small real-time computations while using the existing pilot symbols in the system; thus, no extra pilot overhead is required. In the second stage, we use maximum likelihood estimation to develop a $\mathrm{PH}$ correction scheme. In particular, we formulate the joint estimation of phase noise $(\mathrm{PN})$ and $\mathrm{PH}$ as a maximum likelihood estimation problem. Solving this problem results in the estimation of $\mathrm{PN}$ with the location and magnitude of $\mathrm{PH}$. By applying the proposed correction scheme, the number of affected symbols by $\mathrm{PH}$ is significantly reduced. In particular, the number of remaining erroneous symbols due to PH (if any) is within the error correction capability of modern LDPC codes used 
in the PtPMRL. Numerical results verify the effectiveness of the proposed scheme in detecting and correcting $\mathrm{PH}$.

In our second contribution, we consider one of the key weaknesses of LDPC codes, i.e., the error floor (EF), that they typically exhibit at high signal-to-noise ratios (SNRs). Such an EF is usually attributed to problematic structures known as trapping sets (TS). The overwhelming majority of existing EF estimation schemes consider the case of binary phase shift keying (BPSK) signalling. Unfortunately, these schemes are not readily extensible to estimate the EF of high order LDPC-CMSs used in the PtPMRLs. To provide such a scheme, in this work we use mean-shift importance sampling (MS-IS) to develop a novel EF estimation methodology for high-order pulse amplitude modulation (PAM) LDPC coded systems. First, a computationally efficient graphical-based approach is used to identify the TSs of a given LDPC code. Subsequently, a novel analytical approach is devised to identify the TSs that are likely to have a higher contribution in the EF. These TSs are referred to as potentially dominant TSs (PDTSs). Finally, a new methodology for categorizing the PDTSs into equivalence classes is developed. A representative PDTS of each equivalence class is chosen and an MS-IS framework is devised to obtain the error rate corresponding to each equivalence class. To arrive at the desired MS-IS scheme, we develop an algorithm that invokes the geometry of the constellation to determine the MS value. In contrast with the conventional MS-IS method used in BPSK signalling, in the proposed MS-IS scheme, the MS value is a variable that is determined based on the TS and the transmitted codeword. The computational complexity of the three main steps of our methodology, viz. extracting the PDTSs, determining the MS values, and applying the MS-IS scheme, depends merely on the size of the constellation and the structure of the code, but not on the SNR. Numerical simulations confirm the efficacy and accuracy of the proposed technique at different SNRs.

In our third contribution, we extend our proposed EF estimation scheme to LDPCCMSs using quadrature amplitude modulations (QAM) signalling. QAM is a more bandwidth efficient signalling scheme than the PAM, and it is commonly used in the LDPC-CMSs in the PtPMRLs. The proposed EF estimation can be used as a design guideline in selecting and optimization of the LDPC codes in the LDPC-CMSs used in the PtPMRLs. 
To the love of my life, Neda \&

To my parents 


\section{Acknowledgments}

I would like to express my sincere gratitude to my supervisor, Professor Ramy Gohary for his tremendous support. Working with Prof. Gohary gave me the opportunity of learning technical writing and critical thinking at the same time. I am truly grateful for all the amazing time we spent together working on and discussing every detail of my research. I know that such an opportunity does not exist for many students, and I did not take having that for granted. I would also like to thank him for his friendship, empathy, and great sense of humor.

Foremost, I am extremely grateful to my wife, Neda, for her love, caring, and continuous support to complete my PhD program. I am forever indebted to her for all the sacrifices she made, especially over this past six years. Words cannot express my gratitude and appreciation to her. Also, I would like to express my gratitude to my parents for their love, understanding, prayers and all the sacrifices they made for educating and preparing me for my future.

I would like to thank Huawei Technologies Canada for the generous financial support of this research. Special thanks goes to my manager Dr. Ming Jian, who placed his trust and confidence in my abilities over the past two years, and supported me to complete my research.

I am extending my thanks to all the hard-working staff at the department of Systems and Computer Engineering of Carleton University. I would like to say thanks to Prof. Yvan Labiche, Prof. Paul Keen, and Prof. Gabriel Wainer, who supported me during some of the most difficult times I experienced over the past few years. Also, I thank Ryan Taylor from Research Computing Services to facilitate the use of Carleton RCDC Linux Servers in simulations of this research.

Finally, my thanks go to all the people who have supported me to complete the 
research work directly or indirectly. 


\section{Table of Contents}

Abstract $\quad$ iii

Acknowledgments $\quad$ vi

Table of Contents viii

List of Tables $\quad$ xi

List of Figures $\quad$ xiii

1 Introduction 1

1.1 Motivation . . . . . . . . . . . . . . . . 1

1.2 Oscillator PH in QAM-LDPC Coded Systems . . . . . . . . . . . . . 2

1.3 EF Estimation in LDPC-CMSs . . . . . . . . . . . . . . . . 3

1.4 Organization of Thesis . . . . . . . . . . . . . . 5

2 Background $\quad 6$

2.1 Point-to-point Microwave Radio Links . . . . . . . . . . . . . . 6

2.2 Oscillator Phase Noise . . . . . . . . . . . . . . . . . . . . 7

2.2.1 Wiener Phase Noise Model . . . . . . . . . . . . . . . . . . 8

2.3 Binary Hypothesis Testing . . . . . . . . . . . . . . . . . . 10

2.3.1 Neyman-Pearson Test (NP-BHT) . . . . . . . . . . . . . . . 12

2.4 LDPC Codes . . . . . . . . . . . . . . . . . . . . . . . . . 12

2.4 .1 Trapping Sets . . . . . . . . . . . . . . . 14

2.5 LDPC Coded Modulation Systems . . . . . . . . . . . . . . 14 
3 Mitigation of Phase Hit in PtPMRLs 17

3.1 Introduction . . . . . . . . . . . . . . . . . . . 17

3.2 System Model and Problem Statement . . . . . . . . . . . . . . . . 22

3.2.1 System Model Without PH . . . . . . . . . . . . . 22

3.2.2 $\mathrm{PH}$ Occurrence ..................... 24

3.3 Proposed PH Detection Scheme . . . . . . . . . . . . . 27

3.3.1 Decision Statistic and Likelihood Functions . . . . . . . . . . 27

3.3.2 Characterization of $Z_{0} \ldots \ldots \ldots . \ldots . \ldots 28$

3.3.3 Characterization of $Z_{1}$. . . . . . . . . . . . . . . . . . . . 29

3.3.4 PH Detection Using the NP-BHT . . . . . . . . . . . . . . 32

3.4 Proposed PH Correction Scheme . . . . . . . . . . . . . . . 34

3.4.1 Estimating the PH Location . . . . . . . . . . . . . 35

3.5 Simulation Results and Analysis . . . . . . . . . . . . . . . 38

3.5.1 Performance of PH Detection Scheme . . . . . . . . . . . 38

3.5.2 Performance of PH Correction Scheme . . . . . . . . . . . . 41

3.5.3 BER Results . . . . . . . . . . . . . . . . 45

3.6 Computational Complexity of Proposed PH Mitigation Scheme . . . . 47

4 Error Floor Estimation of PAM-based LDPC-CMS 49

4.1 Application of MC and IS for FER Estimation in LDPC Codes . . . . 54

4.1.1 Comparison Between MC and IS Schemes . . . . . . . . . . . 57

4.1.2 IS and EF Estimation of LDPC Codes With BPSK Signalling 58

4.2 EF Estimation of LDPC Codes with PAM signalling . . . . . . . . 61

4.2.1 Considerations for Evolving From BPSK to PAM Signalling • 62

4.3 Proposed MS-IS scheme . . . . . . . . . . . . . . . 67

4.3.1 Formulation of MS-IS for PAM Systems . . . . . . . . . . . 68

4.3.2 Methodology for Calculating the MS Values . . . . . . . . . . 69

4.3.3 Identifying the PDTSs out of the $N_{a b, j}$ Partitions of $\mathcal{T}_{(a, b)}^{j} \cdots \quad 72$

4.3.4 Summary of the PDTS Extraction Technique . . . . . . . . 76

4.4 Summary of Proposed EF Estimation Scheme . . . . . . . . . . . 78

4.5 Simulation Results and Analysis . . . . . . . . . . . . . . 79

4.5.1 Discussion and Interpretation of Numerical Results . . . . . . 82 
5 Error Floor Estimation of QAM-based LDPC-CMS 88

5.1 Required Modifications in the Proposed EF Estimation Scheme . . . 88

5.1.1 Labelling the BLs . . . . . . . . . . . . . . . . . . . . 89

5.1 .2 Calculating the MS values . . . . . . . . . . . . 89

5.1 .3 Calculating the weight function . . . . . . . . . . . 90

5.2 Simulation Results . . . . . . . . . . . . . . . . 91

5.3 Discussions on Implementing the Proposed EF Estimation Algorithm 94

5.3.1 Number of Required Trials . . . . . . . . . . . . . . . . . . 94

5.3.2 Multiple TS VNs Mapped to One Constellation Point . . . . . 96

6 Conclusion and Future Work $\quad 98$

6.1 Thesis Contributions . . . . . . . . . . . . . . . . . 98

6.2 Future Research Directions . . . . . . . . . . . . . . . . . 100

$\begin{array}{ll}\text { List of References } & 103\end{array}$

$\begin{array}{ll}\text { Appendix A } & 110\end{array}$

A.1 Derivation of $(3.3) \ldots \ldots \ldots \ldots \ldots$

A.2 Derivation of $(3.18) \ldots \ldots \ldots \ldots \ldots$

$\begin{array}{ll}\text { Appendix B } & 115\end{array}$

B.1 Derivation of $(4.30) \ldots \ldots \ldots \ldots \ldots$

B.2 Tables Corresponding to Figs. $4.4-4.6$. . . . . . . . . . . . . 116

$\begin{array}{ll}\text { Appendix C } & 120\end{array}$

C.1 Tables Corresponding to Figs. 5.1 - 5.4 . . . . . . . . . . . . 120 


\section{List of Tables}

3.1 Probability of FA is found using the simulation of $10^{7} \mathrm{PSs}$. The designed $\alpha_{\mathrm{FA}}$ refers to the chosen value for this parameter. The simulated $\alpha_{\mathrm{FA}}$ is the ratio of the FAs over the total PSs found from the simulation. 41

4.1 The empirical values of the threshold $\beta_{\mathcal{T}}^{\text {th }}$ in Algorithm $2 \ldots . . . \quad 80$

4.2 The number of estimated TSs, the relative contribution of a given TS in the total estimated FER, and the total number of trials for the estimation of a TS class are provided for the estimated FERs in Fig. 4.3. 84

4.3 The number of PDTSs and the required trials for estimating the EF of Tanner $(155,64)$ code at an SNR of $27 \mathrm{~dB}$ for 128-PAM signalling is displayed. Three $\beta_{\mathcal{T}}^{\text {th }}$ values are considered. . . . . . . . . . . . . 86

B.1 16-PAM constellation and the bits mapped to each symbol . . . . . . 116

B.2 Error floor estimation of the MacKay $(1008,504)$ LDPC code using $M-\mathrm{PAM}$ signalling. The number of estimated TSs, the relative contribution of a given TS in the total estimated FER, and the total number of trials for the estimation of a TS class are provided in each case. . . . . . . . . . . . . . . . . 117

B.3 Error floor estimation of the WiMAX1 (2016, 1344) LDPC code using $M-\mathrm{PAM}$ signalling. The number of estimated TSs, the relative contribution of a given TS in the total estimated FER, and the total number of trials for the estimation of a TS class are provided in each case. . .

B.4 Error floor estimation of the WiMAX2 $(1152,960)$ LDPC code using $M-\mathrm{PAM}$ signalling. The number of estimated TSs, the relative contribution of a given TS in the total estimated FER, and the total number of trials for the estimation of a TS class are provided in each case. . . 119 
C.1 Error floor estimation of the Tanner $(155,62)$ LDPC code using $M-\mathrm{QAM}$ signalling. The number of estimated TSs, the relative contribution of a given TS in the total estimated FER, and the total number of trials for the estimation of a TS class are provided in each case. . .

C.2 Error floor estimation of the MacKay $(1008,504)$ LDPC code using $M-\mathrm{QAM}$ signalling. The number of estimated TSs, the relative contribution of a given TS in the total estimated FER, and the total number of trials for the estimation of a TS class are provided in each case. . . . . . . . . . . . . . . . . .

C.3 Error floor estimation of the WiMAX1 (2016, 1344) LDPC code using $M-\mathrm{QAM}$ signalling. The number of estimated TSs, the relative contribution of a given TS in the total estimated FER, and the total number of trials for the estimation of a TS class are provided in each

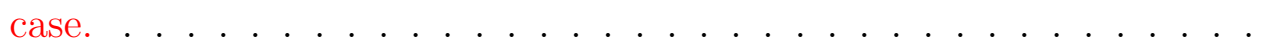

C.4 Error floor estimation of the WiMAX2 $(1152,960)$ LDPC code using $M-\mathrm{QAM}$ signalling. The number of estimated TSs, the relative contribution of a given TS in the total estimated FER, and the total number of trials for the estimation of a TS class are provided in each case. . . 


\section{List of Figures}

2.1 Schematic of a PtPMRL used in the wireless backhaul application. . . 7

$2.210^{6} \mathrm{PN}$ samples according to Wiener model is shown. . . . . . . . . . 9

2.3 SSB-PSD of the PN effect is shown for the time samples shown in Fig. 2.210

2.4 a) The Tanner graph and the parity check matrix of a $(6,3)$ bi-regular LDPC code, b) Part of the Tanner graph of an LDPC code is shown. Three VNs in error are highlighted. The induced subgraph of those VNs is also highlighted. Among the four CNs in the TS, only two are unsatisfied (connected to VNs with dashed lines). The TS is of class

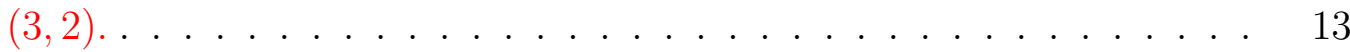

2.5 Block diagram of the LDPC-CMS. . . . . . . . . . . . . . . . 15

3.1 The block diagram of a QAM-LDPC coded system affected by PN and $\mathrm{PH}$ is shown. . . . . . . . . . . . . . . . . . .

3.2 The phase offset of the received symbols within a $\mathrm{PH}$-affected code block. The hit happens in the third PS and influences the phase estimates of all the symbols in the PHPS. . . . . . . . . . . .

3.3 The effect of PH location on the estimation error when using the PSAM PN mitigation is illustrated and compared with a PS without the PH.

a) PH happens at the start of PS, b) PH happens in the middle of PS,

c) PH happens near the end of PS, and d) no PH occurred. . . . . . .

3.4 The block diagram shows the real-time operations of proposed PH detection scheme . . . . . . . . . . . . . . . . 32

3.5 Block diagram of proposed PH correction scheme. . . . . . . . . . . 35

3.6 Block diagram of proposed PH mitigation scheme. . . . . . . . . . 38 
3.7 The theoretical lower bound on the probability of DM is shown for a wide range of FA probability using (3.15) and (3.18) . . . . . . . .

3.8 The theoretical $\operatorname{Pr}(\mathrm{DM})$ is compared with the same parameter, when it is found using the simulation of $10^{7} \mathrm{PSs}$. . . . . . . . . . . .

3.9 CDF of the distance between the detected location and the actual location of the PH. The number of total simulated PSs is $10^{4}$, the constellation is $256-\mathrm{QAM}$, and the $\mathrm{SNR}$ is $26 \mathrm{~dB}$. The size of the $\mathrm{PH}$

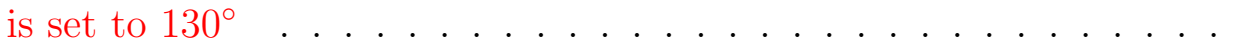

3.10 Effect of PH location on the CDF of the distance between the detected location and the actual location of the $\mathrm{PH}$ is shown for $F_{A}(2)$. Pilot spacing is 50, the number of simulated PSs is $10^{4}$, the constellation is 256-QAM, the $\mathrm{SNR}$ is $26 \mathrm{~dB}$, and the $\mathrm{PH}$ sizes are randomly and

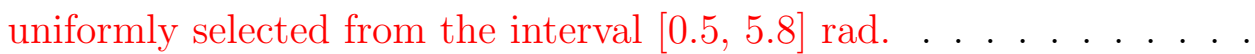

3.11 Effect of PH size on the CDF of the distance between the detected location and the actual location of the $\mathrm{PH}$ is shown for $F_{A}(2)$. Pilot spacing is 50 , the number of simulated PSs is $10^{4}$, the constellation is 256-QAM, the SNR is $26 \mathrm{~dB}$, and the $\mathrm{PH}$ location is set to 25 . . .

3.12 BER of 256-QAM (solid line) and 1024-QAM (dashed line) LDPC CMSs are displayed. Case 1: proposed PH mitigation is applied, Case 2: no PH mitigation is applied. All the curves are drawn in the presence of PN. . . . . . . . . . . . . . . . . . . . 46

4.1 A Gray mapped 8-PAM constellation. . . . . . . . . . . . 63

4.2 Error rate of TSs $T_{1}$ and $T_{2}$ in Example 3. . . . . . . . . . . . 64

4.3 The MC (solid-line) and the MS-IS (dashed-line) simulation results for the Tanner $(155,64)$ LDPC code with $M$-PAM signalling. . . . . . . 81

4.4 The MC (solid-line) and the MS-IS (dashed-line) simulation results for the MacKay $(1008,504)$ LDPC code with $M$-PAM signalling. . . . .

4.5 The MC (solid-line) and the MS-IS (dashed-line) simulation results for the WiMAX1 $(2016,1344)$ LDPC code with $M$-PAM signalling. . . .

4.6 The MC (solid-line) and the MS-IS (dashed-line) simulation results for the WiMAX2 $(1152,960)$ LDPC code with $M$-PAM signalling. . . . 
4.7 Effect of $\beta_{\mathcal{T}}^{\text {th }}$ on the performance of proposed MS-IS technique is illustrated by showing the estimated FER curves corresponding to three threshold values. The solid line and the dashed line show the FER curves corresponding to MC and MS-IS schemes, respectively. . . . .

5.1 The MC (solid-line) and the MS-IS (dashed-line) simulation results for the Tanner $(155,64)$ LDPC code with $M-$ QAM signalling. . . . . . .

5.2 The MC (solid-line) and the MS-IS (dashed-line) simulation results for the MacKay $(1008,504)$ LDPC code with $M$-QAM signalling. . . . . 92

5.3 The MC (solid-line) and the MS-IS (dashed-line) simulation results for the WiMAX1 $(2016,1344)$ LDPC code with $M$-QAM signalling. . .

5.4 The MC (solid-line) and the MS-IS (dashed-line) simulation results for the WiMAX2 $(1152,960)$ LDPC code with $M$-QAM signalling. . . .

A.1 The phase offset on the received signals are decomposed into Gaussian noise component and $\mathrm{PN}$ component. . . . . . . . . . . . . . 111

A.2 Various elements involved in (A.3) are decomposed into radial and tangential components. . . . . . . . . . . . . . . . . 111 


\section{Chapter 1}

\section{Introduction}

\subsection{Motivation}

Over the past two decades, there has been an unprecedented demand for increasing the data rate and achieving higher spectral efficiencies in wireless communication systems. Several solutions are proposed by researchers around the world to fulfil this requirement, e.g., using multiple transmit and receive antennas to build multiple-input multiple-output (MIMO) communication systems, or exploiting abundant bandwith in the high frequency bands in 5 -th generation $(5 \mathrm{G})$ mobile networks [1-4]. These approaches are promising for reinforcing current and building future wireless communication systems. However, the de-facto reality of the current wireless communication infrastructure is that it still relies heavily on the legacy systems, e.g., the point-topoint (PtP) microwave radio links. Beside being the backbone of the current wireless backhaul, the PtP microwave radio links (PtPMRLs) are anticipated to continue to play a crucial role in future mobile networks $[5,6]$. This thesis investigates two challenges in current PtPMRLs.

PtPMRLs are expected to deliver high spectral efficiency at near zero bit error rate (BER). To do so, these systems combine high-order modulation schemes, e.g., high-order quadrature amplitude modulation (QAM), with state-of-the-art forward error correcting (FEC) codes, e.g., low-density parity check (LDPC) codes [7,8]. We refer to this combination as QAM-LDPC coded systems, which forms an instance of LDPC coded modulation system (LDPC-CMS). The focus of this thesis is to address two major challenges in LDPC-CMSs, namely, the oscillator phase hit $(\mathrm{PH})$, and the 
error floor $(\mathrm{EF})$ estimation of LDPC codes. In the following, we briefly introduce each problem and allude to our contribution in solving it.

\subsection{Oscillator PH in QAM-LDPC Coded Systems}

To achieve high spectral efficiency in QAM-LDPC coded systems, we need to increase the modulation size to higher orders. The achievable modulation orders in these systems are limited by several practical constraints specially those imposed by the hardware impairments in the radio frequency $(\mathrm{RF})$ front-end [9-13]. Among various RF impairments, the oscillator phase noise $(\mathrm{PN})$ is one of the key contributors in degrading the performance of PtPMRLs and limiting the modulation order increase [14-18].

The oscillator PN is not the sole source of phase rotation in QAM-LDPC coded systems. On rare occasions, a collection of consecutive received signals are affected by $\mathrm{PH}$, which is typically the result of sudden change (jump) in the frequency of the carrier signal [19-21]. PH causes a large phase change in the affected signals. This large and sudden variation of phase is superimposed by the local oscillator (LO) PN and the overall phase rotation behaves differently from the conventional PN.

In the absence of a $\mathrm{PH}$ mitigation scheme, this hardware impairment results in the link loss in PtPMRLs. Considering that PtPMRLs are expected to meet highest availability of service requirements, e.g., 99.999\% availability, developing a robust $\mathrm{PH}$ mitigation scheme is essential in these systems. $\mathrm{PH}$ mitigation in the literature is commonly performed by modifying the hardware of LO, an inflexible approach, whose effectiveness depends on the LO hardware specifications [19-21].

In this work, an alternative approach to the commonly used hardware-based solutions is adopted by proposing a novel two stage PH mitigation scheme. Our proposed method is composed of a detection stage and a correction stage. We begin by modeling the $\mathrm{PH}$ as a sudden phase change in the $\mathrm{LO}$, which happens at a random time and with a random size. The proposed PH detection scheme exploits binary hypothesis testing (BHT) to find the theoretical optimal threshold for detecting a PH event. The probabilities of giving a false alarm and missing a $\mathrm{PH}$ and their effects on the performance of the system are also investigated. 
The detection of $\mathrm{PH}$ is followed by either a $\mathrm{PH}$ correction stage or by a transmission repeat request. In this thesis, we use the former since it captures a more general approach. The proposed PH correction stage begins with estimating the $\mathrm{PH}$ magnitude. Subsequently, the location of the PH and the PN of the received signals are jointly estimated. The estimated $\mathrm{PN}$ and $\mathrm{PH}$ values are used to de-rotate the received signals before sending them to the decision device. Simulation results verify that the proposed $\mathrm{PH}$ mitigation scheme can be designed such that probability of missing a $\mathrm{PH}$ and giving a false alarm remains as low as $10^{-9}$. Furthermore, numerical results verify that; by using the proposed $\mathrm{PH}$ correction scheme, a significant BER performance improvement is achieved in the QAM-LDPC coded system. To the best of our knowledge, the proposed $\mathrm{PH}$ mitigation scheme is the first to address the $\mathrm{PH}$ problem in the baseband without resorting to hardware modifications, hence offering an attractive solution for the PtPMRLs used in the wireless backhaul of mobile networks.

\subsection{EF Estimation in LDPC-CMSs}

Combining LDPC codes with high order modulations schemes results in serious challenges to the system design. One of these challenges pertain to assessing the high signal-to-noise ratio (SNR) performance of the system [22-24]. In particular, systems using LDPC codes in conjunction with higher-order modulation tend to exhibit an $\mathrm{EF}$, whereby error-rate performance ceases to decay with the increase in the SNR [25]. Since PtPMRLs rely on combining LDPC codes with higher-order modulation, it is necessary to investigate the level of the EF and the SNR at which it occurs. Traditionally, this investigation would be performed using standard Monte Carlo (MC) simulations. However, for practical systems using LDPC codes, EFs arise at frameerror rates (FERs) orders of magnitude less than those that can be accurately assessed using such simulations within a reasonable time frame.

LDPC codes can be represented using the so-called Tanner graphs [26], wherein the encoded bits form the variable nodes (VNs), and the constraints imposed by the LDPC codes form the check nodes (CNs). Tanner graphs can be used to draw insight into the decoding process. In particular, since the length of practical LDPC 
codes is usually large, maximum likelihood detection is computationally infeasible. To circumvent this difficulty, LDPC codes are typically decoded using a close-to-optimal algorithm known as iterative message-passing. In this algorithm, the nodes in the Tanner graph exchange messages that correspond to the reliability of their current decoding decisions, until final decisions are reached. However, particular structures in the Tanner graph, known as Trapping Sets (TS) [25], may cause the decoding progress to cease, yielding undesirable EFs. In fact, in the high SNR regime, failures in the decoder are usually dominated by the errors caused by TSs [25]. Hence, assessing the EF may be achieved by assessing the rate at which the TSs result in decoding failures.

The error rate performance of linear block codes, including LDPC ones, can be estimated using a computationally efficient technique known as importancesampling (IS), see e.g., [27,28]. By invoking the TS structure, this technique can also be used to assess the EF performance of LDPC codes [25, 29]. To do so, the IS technique induces rare error events more frequently through the use of the analytical expressions of the noise distribution to control the frequency with which error events occur. A key element in using the IS technique in assessing EFs is to identify TSs and force them to cause errors at orders of magnitude higher than their actual rate.

In this work, we develop a novel EF estimation technique for higher-order LDPCCMSs. First, a computationally efficient graphical-based approach is used to identify the TSs of a given LDPC code. Subsequently, a novel analytical approach is devised to identify the TSs that are likely to have a higher contribution in the EF. These TSs are referred to as potentially dominant TSs (PDTSs). Finally, a new methodology for categorizing the PDTSs into equivalence classes is developed. A representative PDTS of each equivalence class is chosen and an IS framework is devised to obtain the error rate corresponding to each equivalence class. The computational complexity of the steps of our methodology, viz. extracting the PDTSs, and applying the IS scheme, depends merely on the size of the constellation and the structure of the code, but not on the SNR. Numerical simulations confirm the efficacy and accuracy of the proposed technique at different SNRs 


\subsection{Organization of Thesis}

This thesis is organized as follows. Chapter 2 is dedicated to preliminary background required in the subsequent chapters. Chapter 3 describes the proposed $\mathrm{PH}$ mitigation scheme. In Chapter 4, the proposed EF estimation scheme is presented. In Chapter 5, the proposed EF estimation scheme is extended to QAM-LDPC coded systems, which are widely used in the PtPMRLs. Chapter 6 discusses future work and concludes the thesis. 


\section{Chapter 2}

\section{Background}

\subsection{Point-to-point Microwave Radio Links}

Point-to-point Microwave Radio Links (PtPMRLs) are communication systems operating at frequency bands between 10-42 GHz, and are widely used in the wireless backhaul of mobile networks. The schematic of a PtPMRL used in the wireless backhaul application is displayed in Fig. 2.1. It is observed that the transmitter and the receiver in these systems are installed on the tower and therefore both are fixed. By utilizing parabolic antennas at high altitudes, a clear line of sight (LoS) exists between both sides of the transmission. The RF front-end is attached to the antennas, while the baseband processing is commonly performed in the baseband unit located in a separate room near the tower. The connection between the antennas and the baseband unit is made through optical fiber cables, also known as radio over fiber (RoF).

Due to the fixed location of the transmitter and the receiver and the existing LoS between them in the PtPMRLs, the wireless channel in these systems is stable. Unless the environmental effects, such as rain, influences the fading level, during the normal weather condition the channel can be assumed to be slow and flat. 


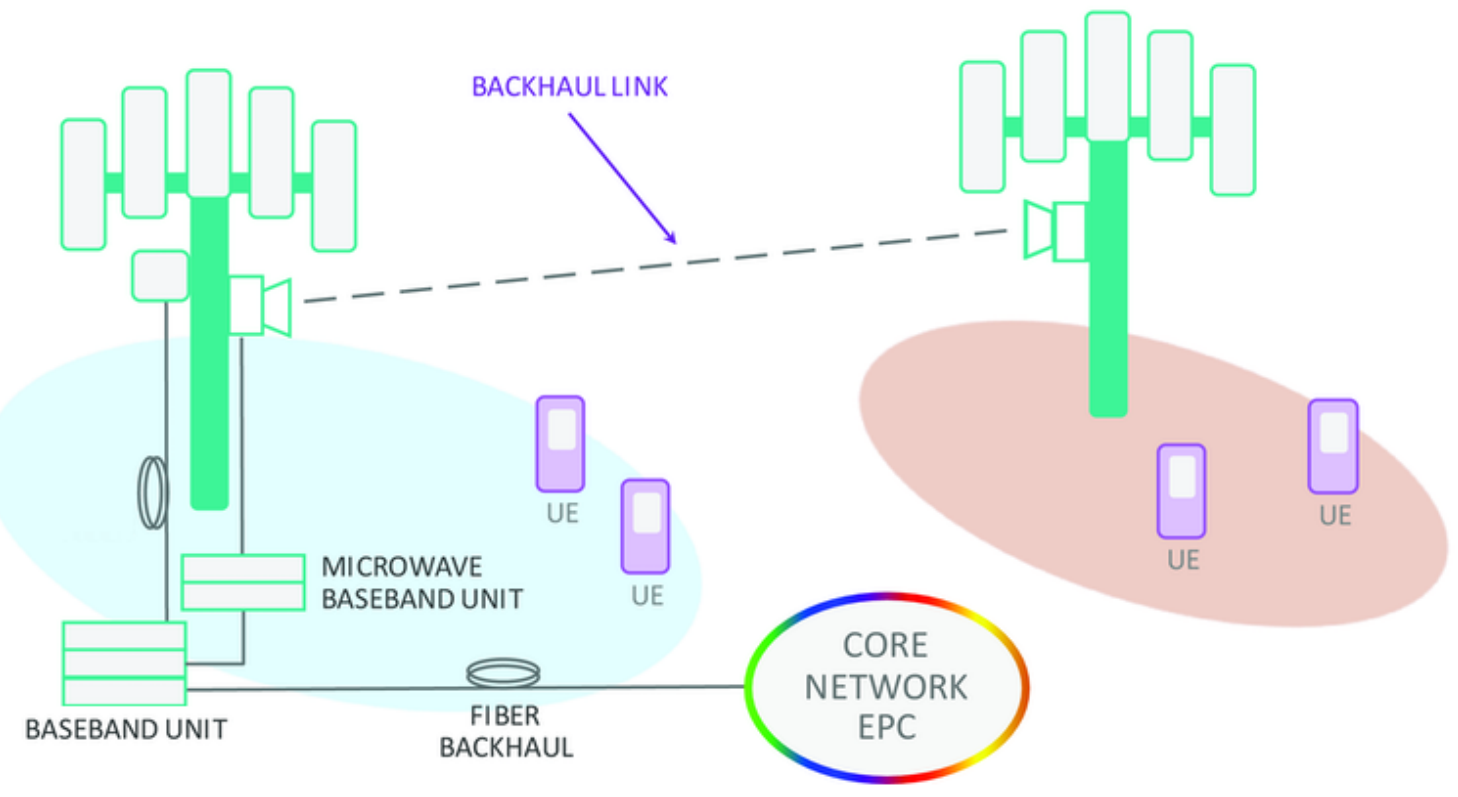

Figure 2.1: Schematic of a PtPMRL used in the wireless backhaul application.

\subsection{Oscillator Phase Noise}

The LO is one of the essential components in every communication system. It generates the carrier signal, which is used for the up-conversion of the baseband signals to carrier frequency or vice-versa. The ideal signal output of an LO at time $t$, denoted by $x(t)$, is a pure sinusoidal tone as follows:

$$
x(t)=e^{j 2 \pi f_{c} t},
$$

where $f_{c}$ is the carrier frequency. We note that it is possible to reconstruct the PSD of the real valued oscillator signal from the PSD of the complex valued signal, $x(t)$ [30]. Due to the noise introduced by different circuit components in the LO, in practice the output signal takes the following form:

$$
\hat{x}(t)=e^{j\left[2 \pi f_{c} t+\phi(t)\right]}
$$

where $\phi(t)$ denotes the oscillator PN. The PN effect is defined as $\theta(t) \triangleq \exp [j \phi(t)]$. Note that without PN, the oscillator will have a power spectral density (PSD) in the form of Dirac delta function centered at $f_{c}$. However, the presence of PN distributes 
power around the carrier frequency. This is the reason that PN is commonly measured at some offset from the carrier frequency. In particular, to measure the PN the normalized single side-band (SSB) power spectral density (PSD) of $\hat{x}(t)$, at offset $f_{0}$ from the carrier frequency is measured. This parameter is denoted by $\mathcal{L}_{\theta}\left(f_{0}\right)$, and is calculated according to the following formula [31]:

$$
\mathcal{L}_{\theta}\left(f_{0}\right) \triangleq \frac{S_{\hat{x}}\left(f_{0}+f_{c}\right)}{P_{\hat{x}}},
$$

where $S_{\hat{x}}(f)$ denotes the SSB-PSD of the oscillator signal at frequency $f$, and $P_{\hat{x}}$ is the average output power of the LO. Note that for the signal contaminated by PN in (2.1), we have $\hat{x}(t)=x(t) \theta(t)$; besides, the signal $x(t)$ is deterministic, which yields

$$
S_{\hat{x}}\left(f_{0}+f_{c}\right)=S_{\theta}(f) * \delta\left(f-f_{c}-f_{0}\right),
$$

where $\delta(\cdot)$ is the Dirac delta function and $*$ denotes the convolution. Equation (2.3) expresses an important result and enables us to measure the oscillator PN through the frequency translation of SSB-PSD of the PN effect, $\theta$.

Different approaches are taken in the literature to derive the mathematical model of PN (e.g., [30-32]). Some of these schemes are useful in the circuit level analysis of the oscillator but are not appropriate for the analysis of the effect of the PN on the performance of digital communication systems. This is because circuit level analysis deals with improving the quality of the LO signal output without investigating the overall effect of impurity of the LO signal on the performance of the communication link. In the following, we introduce a widely used PN model that captures the collective effect of LO impairments beside being suitable for link level analysis.

\subsubsection{Wiener Phase Noise Model}

A commonly used method to accurately model the statistical behavior of the PN originated from a free-running oscillator is the Wiener model [33]. In the Wiener model, the PN $\phi(t)$ is expressed as a Wiener random process. According to properties 


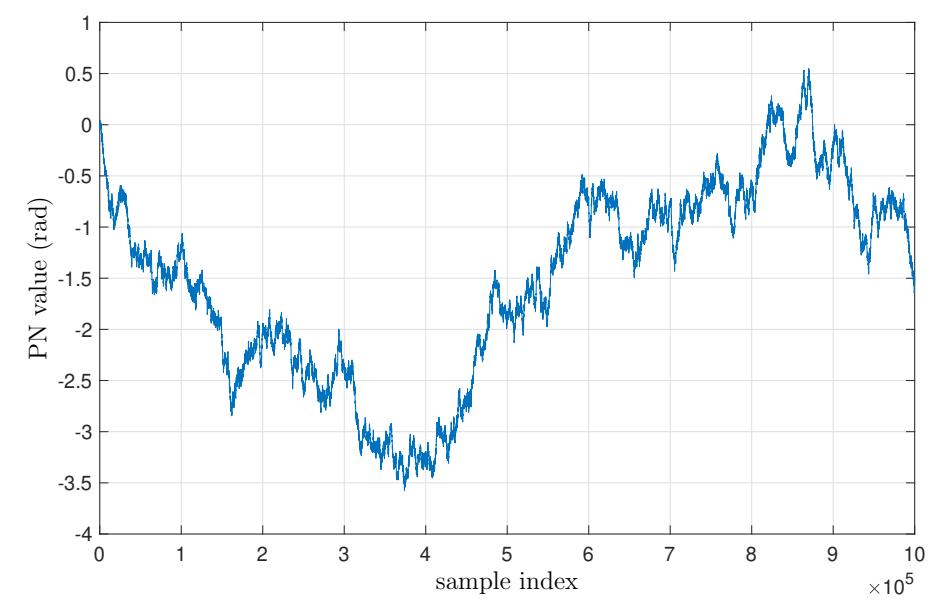

Figure 2.2: $10^{6} \mathrm{PN}$ samples according to Wiener model is shown.

of the Wiener random process, we have

$$
\phi(t) \sim \mathcal{N}\left(0, \sigma_{\phi}^{2} t\right)
$$

where $\sigma_{\phi}^{2}$ is a function of $\mathcal{L}_{\theta}(f)$. From (2.4), it can be readily seen that for two arbitrary time instances, $t_{1}$ and $t_{2}$, we have $\left[\phi\left(t_{2}\right)-\phi\left(t_{2}\right)\right] \sim \mathcal{N}\left(0, \sigma_{\phi}^{2} \Delta t\right)$, where $\Delta t=t_{2}-t_{1}$. In the analysis of the $\mathrm{PN}$ in digital communication systems, we commonly set $\Delta t=T_{s}$, where $T_{s}$ is the symbol interval ${ }^{1}$. In such case, $\sigma_{\phi}^{2} T_{s}$ is defined as the variance of the increment of the process, and the PN corresponding to the $k$-th sample, denoted by $\phi_{k}$, is modeled as follows:

$$
\phi_{k}=\phi_{k-1}+\lambda_{k}
$$

where $\lambda_{k}$ denotes the increment of the process and we have:

$$
\lambda_{k} \sim \mathcal{N}\left(0, \sigma_{\lambda_{\phi}}^{2}\right), \quad \sigma_{\lambda_{\phi}}^{2}=\sigma_{\phi}^{2} T_{s}
$$

In the literature, the variance of the increment, $\sigma_{\lambda_{\phi}}^{2}$, is found using the formula $\sigma_{\lambda_{\phi}}^{2}=2 \pi \beta T_{s}$, where $\beta$ is the 3 -dB linewidth of the $\mathrm{PN}$ [30]. In [34], we derived an

\footnotetext{
${ }^{1}$ We note that $T_{s}$ can be set as the sampling time rather than the symbol interval. In this case, $T_{s}$ will be less than (or equal to) the symbol interval. For simplicity, throughout the thesis we assume one sample per symbol interval. Hence, here $T_{s}$ is identical with the symbol interval.
} 


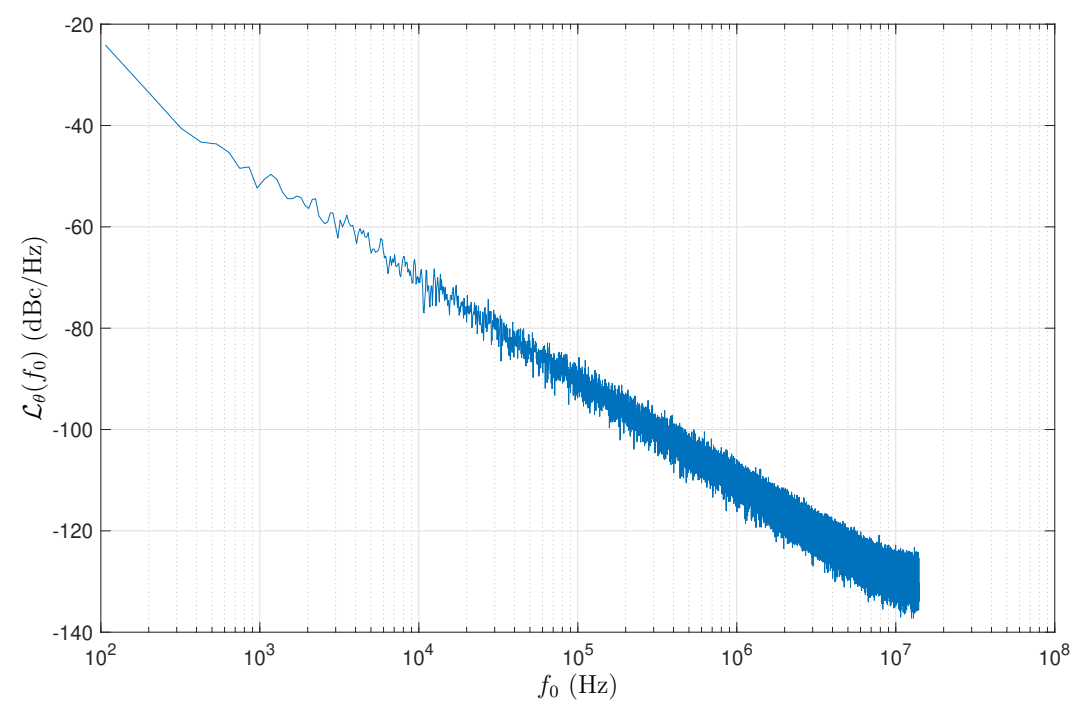

Figure 2.3: SSB-PSD of the PN effect is shown for the time samples shown in Fig. 2.2

alternative novel formula for calculating $\sigma_{\lambda_{\phi}}^{2}$ using PN measurements. An example of PN samples generated according to the Wiener model is provided in Fig. 2.2. The frequency domain characteristics of the PN in Fig. 2.2 is illustrated in Fig. 2.3, where the SSB-PSD is shown at different frequency offsets from the carrier frequency. The simulation parameters for generating these figures are as follows: the PN level at 100 $\mathrm{kHz}$ offset from the carrier frequency is set to $-90 \mathrm{dBc} / \mathrm{Hz}$, the channel bandwidth is $28 \mathrm{MHz}$, and the number of generated samples is $10^{6}$.

\subsection{Binary Hypothesis Testing}

In statistical signal processing, the distinction between two mutually exclusive and collectively exhaustive hypotheses is made by observing a decision statistic. The two hypotheses are denoted by $H_{0}$ and $H_{1}$, where $H_{0}$ corresponds to a situation, when the hypothesis does not hold, while $H_{1}$ corresponds to the opposite situation. The decision statistic is represented by the random variable $Z$. The samples of $Z$, denoted by $z$, are the outcome of random experiments. The decision to accept a hypothesis 
is based on the maximum a posteriori (MAP) rule, and is expressed as follows [35]:

$$
\hat{H}(z)=\arg \max _{H_{i}}\left[\operatorname{Pr}\left(H_{i} \mid Z=z\right)\right]
$$

In (2.7), $\operatorname{Pr}\left(H_{i} \mid Z=z\right)$ is the probability that hypothesis $H_{i}$ holds true conditioned on the observation of $z$ and $\hat{H}(z)$ is the BHT decision. The decision error can be either $\left\{\hat{H}=H_{1} \mid H_{0}\right\}$, which is called the false alarm (FA), or $\left\{\hat{H}=H_{0} \mid H_{1}\right\}$, which is called the detection miss (DM). The optimal decision region is obtained by manipulating (2.7) and applying the Bays rule. The optimal decision region for the BHT is expressed as follows:

$$
\Lambda(z) \underset{H_{1}}{\stackrel{H_{0}}{\gtrless}} \frac{p_{1}}{p_{0}},
$$

where $p_{i}, i \in\{0,1\}$ is the a priori probability of $H_{i}, i \in\{0,1\}$, i.e., $p_{i}=\operatorname{Pr}\left(H_{i}\right), i \in$ $\{0,1\}$, and $\Lambda(z)$ is the likelihood ratio as follows:

$$
\Lambda(z) \triangleq \frac{f_{Z H}\left(z \mid H_{0}\right)}{f_{Z H}\left(z \mid H_{1}\right)}
$$

In (2.9), the likelihood function $f_{Z H}\left(z \mid H_{i}\right), i \in\{0,1\}$ is the PDF of decision statistic $Z$ conditioned on the occurrence of hypothesis $H_{i}, i \in\{0,1\}$. The inequality (2.8) is a likelihood ratio test (LRT). The RHS of (2.8) is called the threshold which is the main design parameter in any BHT. If the two hypotheses are equally likely, the LRT threshold in (2.8) becomes 1 and the MAP test reduces to the maximum likelihood (ML) test. Let us show the decision threshold of BHT with $\gamma$. Note that inequalities $\Lambda(z) \geq \gamma$ and $\Lambda(z) \leq \gamma$ are equivalent to the decisions $\hat{H}(z)=H_{0}$ and $\hat{H}(z)=H_{1}$, respectively. These inequalities are used to calculate the probability of decision error in the BHT. The probability of FA is calculated as follows:

$$
\operatorname{Pr}(\mathrm{FA})=\int_{\Lambda(z) \leq \gamma} f_{Z H}\left(z \mid H_{0}\right) d z .
$$

The probability of DM is calculated as follows:

$$
\operatorname{Pr}(\mathrm{DM})=\int_{\Lambda(z)>\gamma} f_{z H}\left(z \mid H_{1}\right) d z .
$$




\subsubsection{Neyman-Pearson Test (NP-BHT)}

The a priori probabilities of the hypotheses can not be always found. This can cause some inaccuracies in our decision rule which affects adversely on the overall performance of the BHT. To alleviate this problem, Neyman and Pearson proposed an alternative approach in performing the BHT test in which, the knowledge of the $a$ priori probabilities is not necessary [35]. They designed a BHT test to minimize the probability of DM (equivalently maximizing the probability of detection) for a given probability of FA as it is described in the following.

It is shown that among all the possible BHTs under the constraint of $\operatorname{Pr}(\mathrm{FA}) \leq \alpha$ and regardless of a priori probabilities, the LRT results in the least probability of $\mathrm{DM}$ or equivalently, it maximizes the probability of correct detection. The NP-BHT is formulated as follows $[35,36]$. Conditioned on the following FA constraint:

$$
\int_{\Lambda(z) \leq \gamma_{N P}} f_{Z H}\left(z \mid H_{0}\right) d z \leq \alpha,
$$

the threshold to minimize $\operatorname{Pr}(\mathrm{DM})$ is calculated as follows:

$$
\min \operatorname{Pr}(\mathrm{DM})=\int_{\Lambda(z)>\gamma_{N P}} f_{Z H}\left(z \mid H_{1}\right) d z .
$$

The decision regions are found similar to other LRTs and is shown in the following:

$$
\Lambda(z) \underset{H_{1}}{\stackrel{H_{0}}{\gtrless}} \gamma_{N P} .
$$

It can be seen from (2.14) that by replacing the decision statistic, $z$, in the likelihood ratio and comparing the result with the threshold $\gamma_{N P}$ one can decide which hypothesis holds true. The NP-BHT will be used in the proposed PH mitigation scheme in Chapter 3.

\subsection{LDPC Codes}

An $(N, K)$ binary LDPC code is a linear block code of dimension $K$ and block length $N$. For this code, the parity check matrix, $\mathbf{H}$, can be represented by a bipartite 
(a)

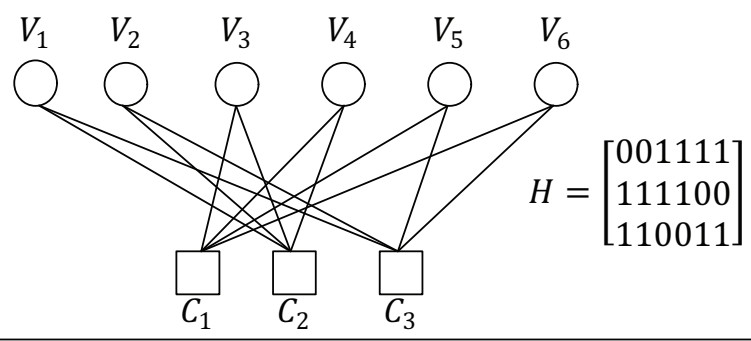

(b)

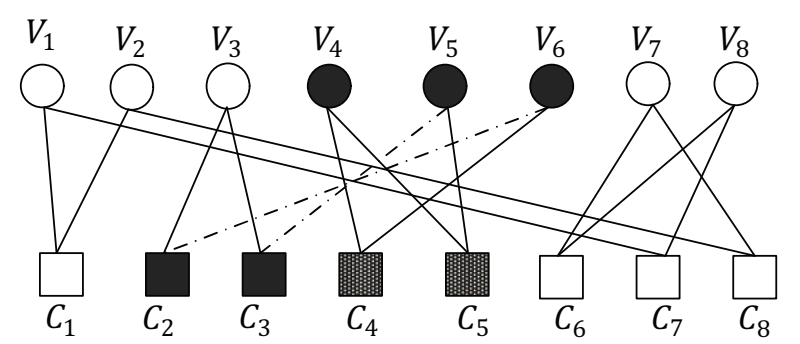

Figure 2.4: a) The Tanner graph and the parity check matrix of a $(6,3)$ bi-regular LDPC code, b) Part of the Tanner graph of an LDPC code is shown. Three VNs in error are highlighted. The induced subgraph of those VNs is also highlighted. Among the four CNs in the TS, only two are unsatisfied (connected to VNs with dashed lines). The TS is of class $(3,2)$.

Tanner graph, $\mathcal{G}$, which consists of $N$ variable nodes (VNs) and $N-K$ check nodes (CNs). A VN which is connected to a $\mathrm{CN}$ by an edge is called the neighbor of that CN. As an illustrative example, the Tanner graph and the corresponding parity check matrix is shown in Fig. 2.4 for a $(6,3)$ LDPC code. The number of edges connected to the $i$-th $\mathrm{VN}(\mathrm{CN})$ is called the variable (check) degree and is denoted by $d_{v}(i)$ $\left(d_{c}(i)\right)$. An LDPC code is bi-regular if all the VNs are of the same degree $d_{v}$ and all the CNs are of the same degree $d_{c}$. If the $\mathrm{VN}$ degrees are the same but the $\mathrm{CN}$ degrees are different, the code is called variable regular. An LDPC code which is neither bi-regular nor variable regular is called irregular [26].

Tanner graphs can be used to draw insight into the decoding process of LDPC codes. In particular, since the length of practical LDPC codes is usually large, maximum likelihood detection is computationally infeasible. To circumvent this difficulty, LDPC codes are typically decoded using a close-to-optimal algorithm known as iterative message-passing. In this algorithm, the nodes in the Tanner graph exchange messages that correspond to the reliability of their current decoding decisions, until final decisions are reached. The reliability of VNs at the beginning of the decoding 
process is provided by the LLR values.

\subsubsection{Trapping Sets}

Given a subset of $a$ VNs from $\mathcal{G}$, an $(a, b)$ TS (also known as class $(a, b)$ TS) is a subgraph of $\mathcal{G}$ consisting of those $a \mathrm{VNs}$ and their neighboring $\mathrm{CNs}$, from which $b$ CNs are unsatisfied (odd degree), cf. Fig. 2.4. The parameter $a$ is referred to as the size of the TS. Since LDPC codes are typically decoded using iterative message passing algorithms, a pragmatic definition of an $(a, b)$ TS is the set of $a$ VNs which remain in error after a maximum number of decoding iterations are performed. In this case, the parameter $b$ is the number of unsatisfied CNs associated with those $a$ bits [25].

In additive white Gaussian noise (AWGN) channels, the error floor of LDPC codes is known to be mainly caused by elementary TS (ETS), i.e., TSs with the CNs of a degree less than or equal to 2 [37-40]. Hence, in the proposed EF estimation scheme in Chapters 4, and 5, all the ETSs within a given class will be considered. We note that using the AWGN channel model to estimate the EF in the LDPC-CMSs used in the PtPMRLs are justified by considering the fact that the fading in these systems is slow and flat (cf. Section 2.1).

To obtain the list of all the ETSs of a given class, we use the graphical-based search algorithm in [40-42]. Therein, it is shown that the ETS structures are layered supersets of short cycles [41], and a small number of other graphical structures within the Tanner graph of the code $[40,42]$. Such property results in a simple search algorithm that is guaranteed to find all ETSs starting from short cycles, and/or other graphical structures.

\subsection{LDPC Coded Modulation Systems}

The block diagram of an LDPC-CMS is shown in Fig. 2.5. The LDPC encoder, receives $K$ bits from the source and generates a codeword of length $N$ at its output. The codewords are denoted by $\mathcal{C}$ and the $i$-th coded bit is denoted by $c_{i}$, where $1 \leq i \leq N$. In this thesis, we assume that the intrinsic random interleaver associated 


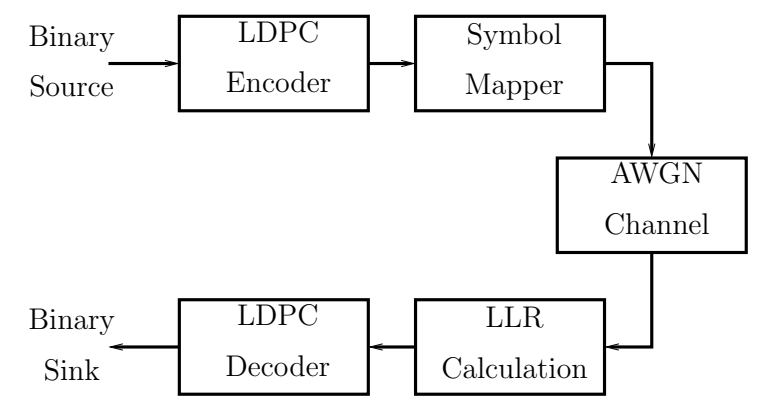

Figure 2.5: Block diagram of the LDPC-CMS.

with the LDPC code is used to eliminate the dependency between adjacent bits of the codewords. Hence, the interleaver block is removed from the block diagram. In the mapper, every $m=\log _{2} M$ bits are Gray-mapped to the symbols from an $M$-ary signal constellation. The number of such symbols for mapping all the bits of an arbitrary codeword, $\mathcal{C}$, to the constellation points are assumed to be $N_{s}$. The $k$-th symbol in this set is denoted by $\left\{s_{k}\right\}_{k=1}^{N_{s}}$. The position of a bit in Gray label of a symbol will be referred to as the bit level (BL). In particular, the 1st BL corresponds to the most significant bit (MSB), which, in PAM and QAM constellations, is the most protected. Analogously, the $m$-th BL corresponds to the least significant bit (LSB), which is the least protected in the aforementioned constellations.

In this thesis, we consider an AWGN channel. The Gaussian noise samples are represented with the zero mean random variable $n$ with variance $N_{0} / 2$ along each signalling dimension, i.e., $n \sim \mathcal{N}\left(0, N_{0} / 2\right)$. The received signals are used to generate the LLRs corresponding to each bit. Using these LLRs, the decoder employs an iterative message passing algorithm, whereby the LLRs are exchanged between VNs and CNs and subsequently updated at the VNs. In practice, these LLRs are quantized and exchanged using a finite number of bits. Upon completing a prescribed number of decoding iterations, the decoder will either converge to a codeword or some of the check equations will remain unsatisfied and decoding fails.

We note that the framework in Fig. 2.5 represents the block diagram of a general LDPC-CMS. In other words, this block diagram can be extended to include further impairments and the corresponding mitigation algorithms. In case of using an $M$ ary QAM constellation, the QAM-LDPC coded system is obtained. In the following chapters, we modify this general block diagram to accommodate for the required 
changes corresponding to the discussed topics. 


\section{Chapter 3}

\section{Mitigation of Phase Hit in PtPMRLs}

\subsection{Introduction}

In the modern wireless mobile backhaul networks, attaining the highest quality of service and availability of service is of great importance. Hence, the high-order QAMLDPC coded systems used in the PtPMRLs are expected to operate at near zero bit error rate (BER) [43]. High order modulations are severely susceptible to the phase rotations in the received signals [7]. The primary cause of phase rotation is the timevarying $\mathrm{PN}$, which is caused by the imperfections in the LO. To face the detrimental effect of PN on the performance of high-order QAM-LDPC coded systems, several PN mitigation schemes are proposed in the literature (e.g., [44] and the references therein). The pilot symbol assisted modulation (PSAM) carrier recovery algorithm is known as an effective PN mitigation method in these systems [14,45-47]. Alternative PN mitigation schemes exist in the literature, e.g., data-aided, and non-data-aided (also known as blind) carrier recovery algorithms [48-52]. Unfortunately, these schemes cannot suppress the PN to the levels required in high-order CMSs. In this chapter, we assume the PSAM is used to mitigate the PN in the LDPC-CMS under consideration.

The oscillator PN is not the sole source of the phase rotation in the high-order CMSs. On the rare occasions, a collection of consecutive received signals are affected by the phase hit (PH) [19]. The PH phenomenon is the result of sudden change (jump) in the frequency of the carrier signal [19,20,53-55]. This jump, also known as the microphonic effect [21], originates from the mechanical interactions between the internal components of the oscillator, e.g., the coefficient of thermal expansion 
mismatch or other physical stresses [56]. Another cause of $\mathrm{PH}$ is the variations in the operating temperature of the LO. The LO is usually mounted outdoors as part of the $\mathrm{RF}$ chain. Specifically, a $\mathrm{PH}$ is more likely to happen when the operating temperature of LO drops below a threshold [55].

The PH causes a large phase change in the affected signals. This large and sudden variation of phase is superimposed by the LO PN. The overall phase rotation behaves differently from the LO PN. Empirical results show that the frequency of the $\mathrm{PH}$ occurrence depends on the LO operating condition, e.g., its operating temperature. Some operating conditions, e.g., extremely cold weather can increase the PH occurrence. In general, the frequency of hits can increase up to one hit per hour, while under normal weather condition this parameter is more likely to be one hit every few (e.g., six) hours.

The PH happens in a random location within one of the pilot sections (PSs). We refer to the PS, where the PH happens therein, as the phase hit pilot section (PHPS). In the PHPS, by definition, the first pilot symbol is not rotated by the $\mathrm{PH}$ while the second pilot symbol is affected by both the PN and the PH. This unusual situation of pilot symbols in the PHPS causes the failure of PSAM scheme in estimating the accurate phase rotations corresponding to the data signals. The direct consequence of such failure is the large phase errors in the PHPS and providing the decision device of the receiver with inaccurate estimate of the received data signals. Note that when using the modern FEC codes, e.g., the LDPC codes or turbo codes, the estimated received signals are used to generate the log-likelihood ratio (LLR) values at the input of the FEC decoder. The inaccuracy of estimated data signals in the PHPS results in generating incorrect LLR values. When using high-order QAM-LDPC coded systems, every received signal represents several coded bits; therefore, the large number of erroneous LLRs originating from the PHPS can cause the decoding failure.

With respect to the rare occurrence of the $\mathrm{PH}$, the importance of this impairment in the overall performance of the system may come into question. While this may be a legitimate concern in many communication systems, e.g., in the mobile access layer, the conditions are different for the backhaul links. The adverse effect of the $\mathrm{PH}$ on availability of service in the PtPMRLs is detrimental and must be avoided. For instance, consider a backhaul link with the BER requirement of $10^{-12}$, and the 
symbol rate of $6 \times 10^{6}$ symbols per second. In the absence of effective $\mathrm{PH}$ and $\mathrm{PN}$ mitigation schemes, a $1 \mathrm{~ms} \mathrm{PH}$ can translate to the same duration of link loss. In other words, it can lead to a maximum of $\frac{10^{-3}}{1 / 6 \times 10^{-6}}=6000$ symbols being in error. Note that the aforementioned BER and symbol rate are equivalent to a maximum of (approximately) 190 symbols errors $^{1}$ annually. Hence, a $1 \mathrm{~ms}$ PH will cause a burst of errors that is well beyond the expected number of errors in a PtPMRL. Therefore, to meet the BER requirement of the wireless backhaul systems, the $\mathrm{PH}$ should be mitigated.

The PH mitigation schemes in the literature are limited to the solutions, which are based on designing better LOs. In other words, the approach has been to avoid/reduce the occurrence of PH $[19,20,53-55]$. The common point between these solutions is the attempt to resolve the issue by modifications in the RF chain, e.g., by adding a heater module to the frequency synthesizer [55]. The main drawback of this approach is that these schemes can not cease the occurrence of $\mathrm{PH}$ completely. Besides, the modifications in the hardware design is necessary, which can limit the adaptability of these techniques to an arbitrary system configuration. Our approach, on the other hand, is based on facing the problem in the baseband by using the digital signal processing tools.

Considering that the $\mathrm{PH}$ results in the burst error, adding a bit interleaver offers an easy solution [57]. The bit interleaver randomizes the errors in a bit stream by dispersing the sequence of the bits. This is commonly achieved by buffering the input stream and un-buffering it in a different order (e.g., in a pseudo-random order) [58]. This imposes an extra latency to both the transmitter and the receiver sides, which is not desired in the backhaul links. As an alternative solution, specific FEC codes, which are capable of correcting burst errors, may be considered. For instance, cyclic codes are known to be capable of correcting burst errors [59]. Unfortunately, the length of the error burst caused by a PH can reach to few hundred bits rendering the required FEC code to correct such burst errors prohibitively complex.

To avoid the excessive complexity and latency associated with the off-the-shelf

\footnotetext{
${ }^{1}$ By calculating the number of transmitted symbols per year, where each symbol is mapped to $m$ bits, we find the maximum number of annual symbol errors corresponding to BER $10^{-12}$ as $\frac{10^{-12} \times 365 \times 24 \times 3600 \times 6 \times 10^{6} \times m}{m} \approx 189.2$.
} 
solutions, in this work, we resort to an ad-hoc approach, which is tailored specifically to face the $\mathrm{PH}$ problem. Regarding the rare occurrence of $\mathrm{PH}$, a complex and computationally expensive detection approach can not be justified. Note that the detection algorithm need to operate continuously to detect any potential $\mathrm{PH}$ events, whereas the correction stage is only triggered when a PH is detected. Consequently, our approach is built upon designing a low cost and low latency detection scheme followed by a robust correction technique. The proposed scheme in this chapter can be readily used to mitigate the $\mathrm{PH}$ in the wireless backhaul links regardless of the initial LO characteristics and without modifying the FEC code or adding an interleaver to the system.

In this chapter, we propose a double stage $\mathrm{PH}$ detection and correction scheme. We begin by modeling the $\mathrm{PH}$ as a sudden phase change in the LO, which happens at a random instance and with a random size. Throughout the thesis, by $\mathrm{PH}$ size we mean the variation in the phase offset of received signals caused by the PH only. This variation will be positive or negative, when the phase offset is increased or decreased by the $\mathrm{PH}$, respectively. This model is considered alongside the effects of the LO PN and the thermal noise on the phase of the received signal. The proposed detection scheme exploits the BHT to find the theoretical optimal threshold for detecting a $\mathrm{PH}$ event. The probabilities of giving a false alarm and missing a PH and their effects on the performance of the system is investigated. The proposed correction stage begins with estimating the PH size. Subsequently, the location of the PH and the PN of the received signals are jointly estimated. The $\mathrm{PH}$ location is used to distinguish between the signals, which are affected by the PN only from those which are affected by both PN and PH. The estimated PN and PH values are used to de-rotate the received signals before sending them to the decision device. Simulation results verify that the proposed detection scheme can be designed such that probability of missing a $\mathrm{PH}$ and giving a false alarm remains as low as $10^{-9}$. On the other hand, numerical results also verify that, by using the proposed correction scheme, the required SNR at a BER of $10^{-5}$ in the QAM-LDPC coded system when contaminated by PN and $\mathrm{PH}$ is approximately $2 \mathrm{~dB}$ more than the required SNR in a system that is when contaminated by PN only. The main contributions of this chapter are as follows: 
- The random behavior of the PH is modeled in a high-order coded QAM system, which is affected by time-varying LO PN. The probability distribution function (PDF) of phase difference between the pilot symbols are derived at the presence of $\mathrm{PH}$.

- A likelihood ratio test is designed for the detection of PH. In particular, the NP-BHT $[35,36]$ is chosen for this task due to the independence of the test from a priori statistical behavior of the $\mathrm{PH}$. The optimal threshold of NP-BHT is analytically derived.

- A joint PN mitigation and PH correction algorithm is proposed. Following the estimation of PH size, the proposed scheme estimates the location of PH. Using this information, the PN is also estimated and the effects of $\mathrm{PN}$ and $\mathrm{PH}$ are jointly removed from the affected symbols.

Considering that the main scope of this work is on mitigating the effect of $\mathrm{PH}$ on the system, it is essential to clarify the difference between the $\mathrm{PH}$ and other similar impairments. By similar impairments we mean the ones, which cause sudden and large phase offsets on the received signal. To the best of our knowledge, these include the frequency jump (see [60] and the references therein) and the cycle slip (see [61] and the references therein).

The frequency jump is a common issue in the atomic clocks, which are used in the global navigation satellite system (GNSS). The characteristics of the frequency jump, e.g., the jump amplitude and the required detection time is relevant to the specific GNSS application. These requirements are different from what is imposed by the wireless mobile backhaul scenario, e.g., in atomic clocks a sudden frequency change in the range of $10^{-4} \mathrm{~Hz}$ is considered a jump, while the same jump will translate to a negligible phase change in the wireless backhaul scenario.

The cycle slip, on the other hand, pertains to the symmetry angle of the constellation. Some constellations are invariant under certain rotation angles, e.g., a square QAM constellation is invariant under the rotations of $k \frac{\pi}{2} \mathrm{rad}$, where $k$ is an integer. The cycle slip can be considered a special case of the $\mathrm{PH}$, when the hit size is equal to the symmetry angle of the constellation. Since the PH size can take any arbitrary 
value depending on the LO design and the operating condition, we refrain to limit our investigations to specific rotation angles. Instead, we propose a scheme, which mitigates the $\mathrm{PH}$ over a wide range of hit sizes.

Rest of this chapter is organized as follows: Section. 3.2 describes the general structure of the system under the study. This is followed by explaining the mechanism of PH occurrence and its mathematical model. The proposed PH detection scheme is explained in Section 3.3. In Section 3.4 the proposed PH correction technique is described. In Section 3.5, the proposed algorithms are implemented on a high-order QAM-LDPC coded system and the numerical results are provided.

Throughout this chapter, a Gaussian random variable, $X$, with mean $\mu_{X}$ and variance $\sigma_{X}^{2}$ is denoted by $X \sim \mathcal{N}\left(\mu_{X}, \sigma_{X}^{2}\right)$. Similarly, $W \sim \mathcal{U}(a, b)$ represents the uniform random variable with the $\operatorname{PDF} f_{W}(w)=\frac{1}{b-a}[u(w-a)-u(w-b)], \quad \forall w \in$ $\mathcal{R}, b>a$, where $\mathcal{R}$ is the set of real numbers and $u(x)$ is the Heaviside step function. The error function $\mathrm{Q}(x)$ is defined as $\mathrm{Q}(x) \triangleq \int_{t>x} \frac{1}{\sqrt{2 \pi}} \exp \left(-\frac{t^{2}}{2}\right) d t$.

\subsection{System Model and Problem Statement}

In this section, we describe the system model that is used in our PH mitigation scheme. The role of each block is Fig. 3.1 is explained both during the normal operation of the system, and the time of $\mathrm{PH}$ occurrence.

\subsubsection{System Model Without PH}

The block diagram of the QAM-LDPC coded system, considered in this chapter, is shown in Fig. 3.1. The data bits generated by a random source are encoded and mapped to the symbols from a high-order QAM constellation. The data symbols and the pilot symbols, used for the PN and PH mitigation, are multiplexed together. A digital to analogue converter (DAC) block applies the pulse shaping filter and converts the symbols to the analogue signals. The transmit LO is used to up-convert the signals to the high frequency. The modulated symbols are transmitted over an AWGN channel. We assume the fading is slow and flat. This is a valid assumption for the fixed PtPMRLs, which has the transmitter and the receiver mounted on the top of 


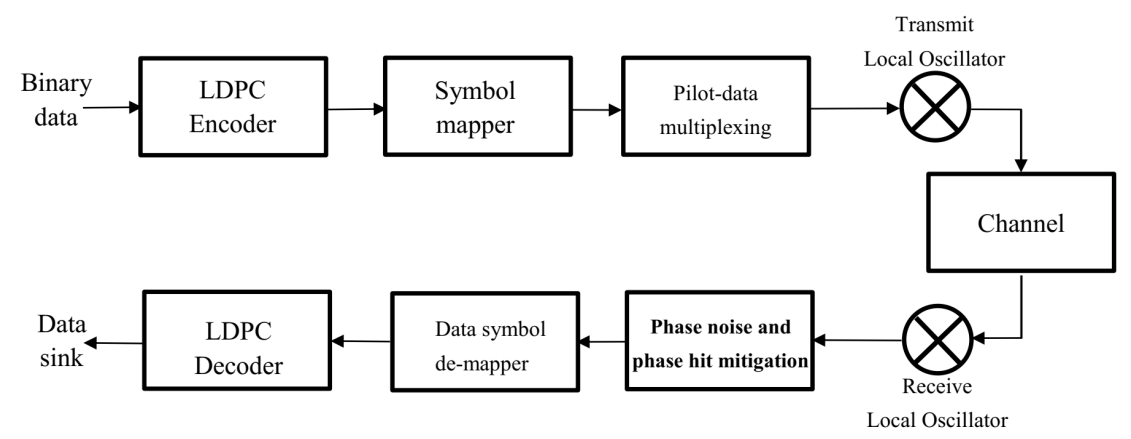

Figure 3.1: The block diagram of a QAM-LDPC coded system affected by PN and $\mathrm{PH}$ is shown.

tall buildings and the LoS exists between both ends of transmission. At the receiver, the receive LO is expected to generate a signal, ideally, identical to the transmitter's carrier waveform; nevertheless, due to the imperfections in the commonly used CMOS based LOs, this will not happen and the generated signal has a small phase difference with the incoming carrier's signal. This indicates the existence of PN problem. The generated carrier signal by the receive LO is used for the down-conversion of received signals. In addition to the $\mathrm{LO} \mathrm{PN}$, in the case of $\mathrm{PH}$ occurrence, the phase of received signals will experience another large variation. The $\mathrm{PH}$ occurrence and its effect is explained in Section 3.2.2.

Following the down-conversion, a matched filter is applied and the signals are sampled every $T_{s}$ seconds, where $T_{s}=\frac{1}{f_{s}}$ and $f_{s}$ is the symbol rate. The sampled signals are passed through a PN removal block. In this work, the commonly used $\mathrm{PN}$ removal block is replaced with the $\mathrm{PN}$ and $\mathrm{PH}$ mitigation block, in which the $\mathrm{PH}$ detection operates continuously. The $\mathrm{PH}$ detection scheme divides the system operating modes into the normal and the $\mathrm{PH}$ operating modes. By normal operating mode, we mean the system operation, when no $\mathrm{PH}$ has happened or when the $\mathrm{PH}$ is occurred but corrected. By the PH operating mode, we mean a PH has happened but have not been detected or corrected. During the normal operating mode, the conventional PN mitigation scheme, e.g., PSAM is used [45]. Our PSAM implementation is based on the linear interpolation between the consecutive pilot signals to estimate the PN. During the PH operating mode, the proposed joint PN and PH correction scheme is used to mitigate the effects of PN and PH from the received signals. Upon 


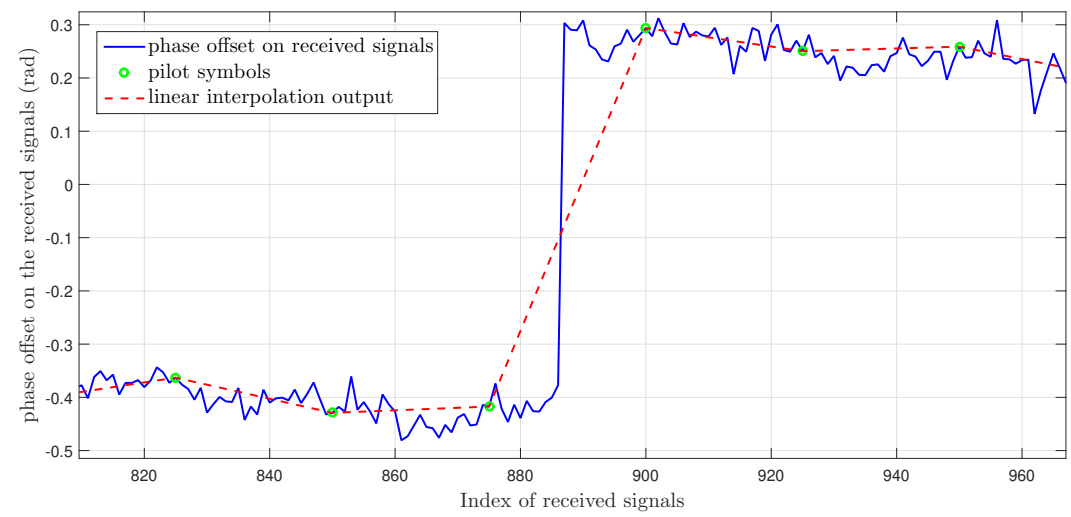

Figure 3.2: The phase offset of the received symbols within a PH-affected code block. The hit happens in the third PS and influences the phase estimates of all the symbols in the PHPS.

mitigating the $\mathrm{PN}$ and $\mathrm{PH}$, the estimated received signals are used for generating the channel LLRs. The LLRs are sent to the LDPC decoder, which- conditioned on the convergence- recovers the transmitted bits. The baseband samples of the received signals before the PN/PH removal are represented as follows:

$$
r=s e^{j \phi}+n,
$$

where $r$ and $s$ are the received and the transmitted signals, respectively, and $n$ is a circular symmetric complex Gaussian random variable with mean 0 and variance $\sigma_{n}^{2}$ along each dimension. The Gaussian noise samples are assumed to be independent and identically distributed (i.i.d.) The parameter $\phi$ represents the phase rotation of received signal. Depending on the operating mode, the phase rotation $\phi$ is composed of various components. These components are discussed in Section. 3.3.1. For the received signal, $r$, corresponding to the transmitted symbol, $s$, we define the phase of $\frac{r}{s}$ as the phase offset on $r$, i.e., $\varphi \triangleq \angle \frac{r}{s}$.

\subsubsection{PH Occurrence}

As it was explained in Section 3.1, occasionally, due to various factors such as the mechanical stresses, the phase of oscillator experiences a sudden large jump. This is reflected as a large phase rotation on the received signals after the PH instance. In 

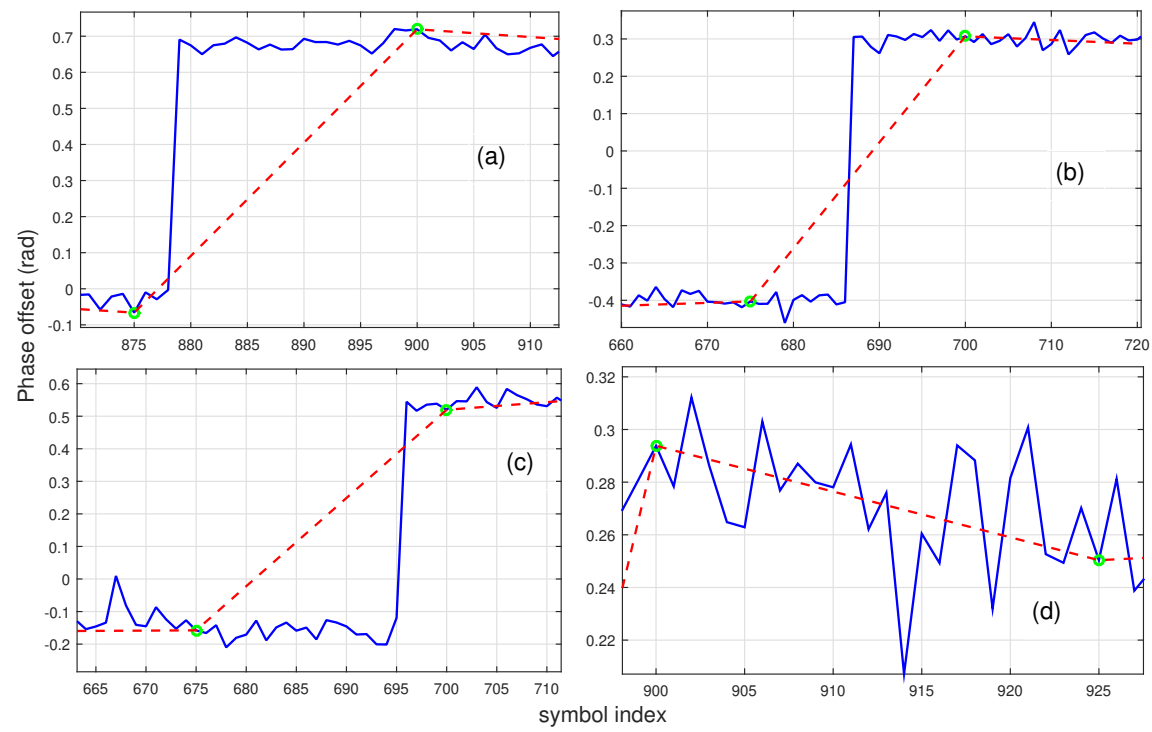

Figure 3.3: The effect of PH location on the estimation error when using the PSAM $\mathrm{PN}$ mitigation is illustrated and compared with a PS without the PH. a) $\mathrm{PH}$ happens at the start of PS, b) PH happens in the middle of PS, c) PH happens near the end of PS, and d) no PH occurred.

Fig. 3.2 the phase offset on the received signals are displayed during 5 consecutive PSs. These phase offsets include the LO PN, the rotational effect of Gaussian noise on the signal and the PH (from the point it starts). The third PS in this figure is the PHPS. It is observed that the pre-hit pilot in the PHPS does not have any extra phase offset (other than the effect of AWGN and LO PN). In contrast, the post-hit pilot in the PHPS has a large phase offset due to the PH occurrence. This makes the linear interpolation error very large and beyond the tolerable values of the high-order modulations. The PSs immediately before and after the PHPS are referred to as the pre-hit PS and the the post-hit PS, respectively.

The PSs following the PHPS can cope with the PH problem since both of the involved pilots in the interpolation have similar large phase offsets and thus they generate valid estimates. This means that PH operating mode lasts only one PS; nevertheless, in a typical high-order CMS, the adverse effect of this individual PS on the performance of the system can be significant. The following example illustrates this effect. 
Example 1 A wireless backhaul link uses 1024-QAM, and the symbols are assumed to be protected by an LDPC code with the block length 4032 and the code rate 7/8. The number of symbols in each code block is $\left\lfloor\frac{4032}{10}\right\rfloor=403$ (where $\lfloor x\rfloor$ is the smallest integer less than or equal to $x$ ). This dense constellation is prone to the PN severely. We use the PSAM with the pilot spacing $P=25$ to mitigate the PN effect; thus, a pilot and 24 data symbols after that make one PS. Notice that 403 is the total number of the symbols in the code block, 15 of which are the pilots and 388 are the coded data symbols. The symbol rate is assumed to be $6 \mathrm{MHz}$. We further assume a PH happens at a random location within the received code block. The $L O P N$ level is $-95 \mathrm{dBc} / \mathrm{Hz}$ at $100 \mathrm{kHz}$ offset from the carrier frequency, the SNR is $32 \mathrm{~dB}$ and the size of the jump is assumed to be $\pi / 6$ rad. In Fig. 3.2, we have shown the curves corresponding to the phase offsets on the signals within a code block before, during, and after the PH according to this system configuration. This figure shows how a PH can cause a burst of errors due to the loss of all the symbols within the PHPS. The large PN estimation errors in the PHPS is evident, which yields de-rotation of the symbols with wrong values. In this example, around $25 \times 10$ bits will be affected and with a larger pilot spacing the number increases even further. The same scenario is repeated for the different PH locations within the PHPS, i.e., near the pre-hit pilot, in the middle of PHPS and near the post-hit pilot. The phase offsets of the received signals in these scenarios are displayed in Fig. 3.3. The large estimation error exists regardless of the hit location; however, a PH at the middle of the PHPS (Fig. 3.3 (b)) causes PSAM estimation error on all the symbols in the PHPS, while if the PH happens close to the borders of the PHPS (Fig. 3.3 (a),(c)), there is a small portion of symbols with smaller estimation errors. Fig.3.3(d) is drawn to illustrate the PN estimation during the normal operating mode.

The large estimation error of the PSAM scheme during the PH operating mode signifies the requirement of having a robust PH removal scheme. In the following sections, our proposed method to mitigate the PH effect is explained. 


\subsection{Proposed PH Detection Scheme}

The proposed $\mathrm{PH}$ detection scheme is based on the idea of exploiting the existing information of the pilot symbols to identify the $\mathrm{PH}$ occurrence. In other words, the detection will use the phase information of the pilots, which are already available since those are necessary for the interpolation in PSAM PN mitigation. The phase information of the pilots serve as the input of the designed detection scheme. The actual detection algorithm, however, relies on the NP-BHT scheme [35]. In Section 2.3.1 we briefly reviewed the NP-BHT. To apply the NP-BHT in the detection of PH, we need to design the different elements in the test. These elements include the decision statistic random variable and the underlying likelihood functions. Upon deriving the required likelihood functions, the optimal threshold in the NP-BHT is calculated and the test can be used for the PH detection. These steps are explained in the following.

\subsubsection{Choosing the Decision Statistic and Derivation of Like- lihood Functions}

To apply the NP-BHT in the detection of $\mathrm{PH}$, we propose to use the statistical behavior of pilots phase offset. Note that phase offset values of the pilots are available to the receiver because of its application in the PSAM PN mitigation. The decision statistic that we choose is a function of this phase offset. In particular, we define the absolute value of difference between the phase offset of consecutive pilots as our decision statistic. The decision statistic, $Z_{i}$, is expressed as follows:

$$
Z_{i} \triangleq\left|\Delta \varphi_{i}\right|=\left|\varphi_{i}\left(t_{K+1}\right)-\varphi_{i}\left(t_{K}\right)\right| \quad, i \in\{0,1\} .
$$

In (3.2), $t_{K}$ is the time instance when the $K^{\prime}$ th pilot is sampled at the output of detector. The indices $i=0$ and $i=1$ represent the normal operating mode and $\mathrm{PH}$ operating mode, respectively. In the following, we explain the different components of $Z_{i}$ and derive the required likelihood functions. We begin with considering the phase offset of the pilot symbols. In the following, the mathematical model for the phase offset of pilots are characterized for both operating scenarios. 


\subsubsection{Characterization of $Z_{0}$}

During the normal operating mode, the phase offset of pilots consists of two elements. We show these elements with two random variables $\varphi_{\omega}$ and $\varphi_{\mathrm{PN}}$. Considering that the normal operating mode is represented by $H_{0}$ the total phase offset during this mode is denoted by the random variable $\varphi_{0}$. Clearly, $\varphi_{0}=\varphi_{\omega}+\varphi_{\mathrm{PN}}$, in which the parameter $\varphi_{\omega}$ is the effect of the AWGN on the phase offset and $\varphi_{\mathrm{PN}}$ originates from the LO PN. Considering that the AWGN effect can be decomposed to the tangent and the radial components, it is shown in Appendix A.1 that the random variable $\varphi_{\omega}$ can be approximated (for the relatively high SNR values) by a Gaussian distribution as follows:

$$
\varphi_{\omega} \sim \mathcal{N}\left(0, \mathcal{E}\left\{|n|^{2} / 2\right\} \cdot \mathcal{E}\left\{\left|s_{p}\right|^{-2}\right\}\right)
$$

In (3.3), $\mathcal{E}\{\cdot\}$ is the expected value, and $s_{p}$ is used to display the pilot symbols.

The second parameter in $\varphi_{0}$ is the LO PN, which is modeled by the Wiener random process. The variance of $\varphi_{\mathrm{PN}}$ depends on the PN level and the time difference with a certain reference point, i.e., $\varphi_{\mathrm{PN}} \sim \mathcal{N}\left(\mu, \sigma_{L}^{2} t\right)$. The mean value $\mu$ is irrelevant in our calculations as it will be explained in the following. The parameter $\sigma_{L}^{2}$ is calculated using $\sigma_{L}^{2}=4 \pi^{2} f_{0}^{2} \times 10^{\frac{L}{10}}$, where $L$ is the PN level at frequency offset $f_{0}$ from the carrier frequency [34]. The parameter $t$ can be any time instance, e.g., when a pilot symbol is sampled at the output of the matched filter. This results in $t=k T_{s}$, where $k$ is an integer corresponding to the index of a pilot sample. According to (3.2) and the aforementioned discussion, during the normal operating mode we have:

$$
Z_{0}=\left|\left[\varphi_{\omega}\left(t_{K+1}\right)-\varphi_{\omega}\left(t_{K}\right)\right]+\left[\varphi_{\mathrm{PN}}\left(t_{K+1}\right)-\varphi_{\mathrm{PN}}\left(t_{K}\right)\right]\right| .
$$

In (3.4), $\left[\varphi_{\omega}\left(t_{K+1}\right)-\varphi_{\omega}\left(t_{K}\right)\right]$ is the subtraction of two independent Gaussian random variables, which is another Gaussian random variable. On the other hand, according to the definition of Wiener random process (which the PN is modeled with), the second subtraction in (3.4), i.e., $\left[\varphi_{\mathrm{PN}}\left(t_{K+1}\right)-\varphi_{\mathrm{PN}}\left(t_{K}\right)\right]$, can also be modeled by a Gaussian random variable. Since the time frame between the two consecutive pilots is $t_{K+1}-t_{K}=P T_{s}$ ( $T_{s}$ being the symbol interval and $P$ the pilot spacing), for the second subtraction, we have $\left[\varphi_{\mathrm{PN}}\left(t_{K+1}\right)-\varphi_{\mathrm{PN}}\left(t_{K}\right)\right] \sim \mathcal{N}\left(0, \sigma_{L}^{2} P T_{s}\right)$. This results in 
$Z_{0}$ being the absolute value of the Gaussian random variable, $\Delta \varphi_{0}$, i.e., $Z_{0}=\left|\Delta \varphi_{0}\right|$ where $\Delta \varphi_{0} \sim \mathcal{N}\left(0, \sigma_{P}^{2}\right)$. The parameter $\sigma_{P}^{2}$ is calculated as follows:

$$
\sigma_{P}^{2}=2 \mathcal{E}\left\{|n|^{2} / 2\right\} \cdot \mathcal{E}\left\{\left|s_{p}\right|^{-2}\right\}+\sigma_{L}^{2} P T_{s} .
$$

Under the constraint of $\Delta \varphi_{0} \neq 0$, the PDF of $Z_{0}$ is derived by letting $i=0$ in the following relationship:

$$
f_{(Z H)}\left(z \mid H_{i}\right)=\left[f_{\Delta \varphi_{i}}(z)+f_{\Delta \varphi_{i}}(-z)\right] u(z) \quad, \quad i=0,1,
$$

where $f_{\Delta \varphi_{0}}(z)$ is a Gaussian PDF with mean 0 and variance $\sigma_{P}^{2}$. Due to the symmetry of Gaussian PDF, the likelihood function in the normal operating mode is expressed as follows:

$$
f_{(Z H)}\left(z \mid H_{0}\right)=\sqrt{\frac{2}{\pi \sigma_{P}^{2}}} \exp \left\{\frac{|z|^{2}}{2 \sigma_{P}^{2}}\right\} u(z)
$$

\subsubsection{Characterization of $Z_{1}$}

For the $\mathrm{PH}$ operating mode, we need to include an extra random variable to represent the effect of PH on the pilot's phase offset. As it was mentioned in Section 3.2.2, the $\mathrm{PH}$ results in a sudden jump in the phase of received signals. Let $t_{\mathrm{PH}}$ represent the random time instance, when the $\mathrm{PH}$ initiates. We assume the time instances corresponding to the pilot symbols in the PHPS are denoted by $t_{K}$ and $t_{K+1}$, respectively; thus, we have $t_{K}<t_{\mathrm{PH}} \leq t_{K+1}$.

To reflect the effect of $\mathrm{PH}$ on the phase offset, we define the random variable $\varphi_{\mathrm{PH}}$. This gives that in the $\mathrm{PH}$ operating mode, the total phase offset is composed of three elements and we define the random variable $\varphi_{1}$ to represent that, i.e., $\varphi_{1}=$ $\varphi_{\omega}+\varphi_{\mathrm{PN}}+\varphi_{\mathrm{PH}}$. It is worth noting that the subscript 1 is chosen since the $\mathrm{PH}$ operating mode is represented by hypothesis $H_{1}$. In the PSs after the PHPS, the PH exists on both pilots, which are involved in the linear interpolation module. For the PHPS, however, the phase offset of the preceding pilot does not have any PH effect while the pilot after the $\mathrm{PH}$ instance experiences the large rotation due to the $\mathrm{PH}$. Because of this effect, the phase offset difference in the PHPS follows a different distribution.

To model the difference between the phase offsets of the two consecutive pilots in 
the PHPS, we let $Z_{1}=\left|\Delta \varphi_{1}\right|$. Hence, for the random variable $Z_{1}$ we have:

$$
\begin{aligned}
Z_{1} & =\mid\left[\varphi_{\omega}\left(t_{K+1}\right)-\varphi_{\omega}\left(t_{K}\right)\right]+\left[\varphi_{\mathrm{PN}}\left(t_{K+1}\right)-\varphi_{\mathrm{PN}}\left(t_{K}\right)\right] \\
& +\left[\varphi_{\mathrm{PH}}\left(t_{K+1}\right)-\varphi_{\mathrm{PH}}\left(t_{K}\right)\right] \mid .
\end{aligned}
$$

The first two subtractions in the RHS of (3.8) are found similar to normal operating mode. The third subtraction can be viewed as the total phase rotation caused by the $\mathrm{PH}$ between the two consecutive pilots. This is a random value, for which we have no prior knowledge. The same argument applies to the time instance within the PHPS, when the PH occurs. In this work, we consider the uniform random variable to model both the location and also the size of the PH. This choice is due to the fact that we have no prior knowledge about the time and the size of $\mathrm{PH}$. Choosing other distributions does not affect the general framework of the proposed scheme. However, it requires the derivation of likelihood functions based on the chosen PDF. The location of the PH between the two consecutive pilots (shown by the time label $t_{\mathrm{PH}}$ ) can be anywhere starting from the first symbol after the pilot, $s_{p}^{(K)}$, up to the next pilot,

$s_{p}^{(K+1)}$. To include the randomness of $t_{\mathrm{PH}}$, we define the discrete uniform random variable, $\tau$, as an integer between 1 and $P$. The $\mathrm{PH}$ occurrence time is expressed as $t_{\mathrm{PH}}=t_{K P+\tau}$ and $\operatorname{Pr}(\tau=i)=\frac{1}{P} \quad \forall i \in\{1,2, \ldots, P\}$.

Regarding the size of the $\mathrm{PH}$, we can use another uniform distribution but this time the size is a continuous random variable. Considering that the $\mathrm{PH}$ affects the phase of the signal, its effective minimum and maximum value varies between 0 and $2 \pi$; nevertheless, the small values of PH need not to be considered since those will not cause large rotations. Let us show the minimum and the maximum of the PH's size by $d_{m}$ and $d_{M}$, respectively. In the following, we describe how to choose $d_{m}$ and $d_{M}$.

\section{Choosing $d_{m}$ and $d_{M}$}

Let $V$ be the random variable, which indicates the difference between the phase offsets of 2 consecutive received signals during the normal operating mode. Let $v_{\max }$ represent the maximum of $|V|$. By definition, the samples of $V$ will not become greater than $v_{\max }$ unless a $\mathrm{PH}$ happens. In other words, if the difference between the 
phase offsets of 2 consecutive received signals become greater than $v_{\max }$, with a high probability a $\mathrm{PH}$ has happened, which results in $d_{m} \approx v_{\max }$. Similarly, we can choose the maximum of the PH's size, i.e., we let $d_{M} \approx 2 \pi-v_{\max }$.

To calculate $v_{\max }$ we consider the standard deviation of $V$. This is justified by the fact that the standard deviation can be used as a measure, which indicates the typical values of a random variable. Furthermore, it is easy to see that for an arbitrary Gaussian random variable $X \sim \mathcal{N}\left(0, \sigma^{2}\right)$, we have $\operatorname{Pr}(|X| \geq 8 \sigma) \approx 10^{-15}$. This gives that the absolute value of all the samples of $X$ are less than $8 \sigma$ with probability $1-10^{-15}$. We use this argument and let $|X|_{\max } \approx 8 \sigma$. Hence, we need to calculate $\sigma_{V}$, i.e., the standard deviation of $V$. To do so, we consider a hypothetical case, where $P=1$ and the pilot set is equal to all the constellation points. This assumption is used to calculate $\mathcal{E}\left\{\left|s_{p}\right|^{-2}\right\}$ for this hypothetical scenario. From this discussion we choose $d_{m}=8 \sigma_{V}$ and $d_{M}=2 \pi-8 \sigma_{V}$, where by using (3.5), $\sigma_{V}=\left(2 \mathcal{E}\left\{|n|^{2} / 2\right\} \cdot \mathcal{E}\left\{|s|^{-2}\right\}+\sigma_{L}^{2} T_{s}\right)^{1 / 2}$. Upon choosing $d_{m}$ and $d_{M}$, we can form the uniform random variable $D$, which represents the $\mathrm{PH}$ size as follows:

$$
D \sim \mathcal{U}\left(d_{m}, d_{M}\right) \quad, \quad d_{m}=8 \sigma_{V} \quad, \quad d_{M}=2 \pi-8 \sigma_{V} .
$$

By using the statistical models of $\Delta \varphi_{0}, D$ and $\tau$ we derive the probabilistic model of $\Delta \varphi_{1}$. Note that for a given hit location and hit size, i.e., for $\left\{\Delta \varphi_{1} \mid D=x, \tau=i\right\}$ we have the same random variable as $\Delta \varphi_{0}$ with a different mean only. This conveys the idea of using the chain rule and integrating out the unnecessary random variables to find the desired marginal PDF, i.e., the PDF of $\Delta \varphi_{1}$. Since the PH is independent of the AWGN and the LO PN, the conditional random variable $\left\{\Delta \varphi_{1} \mid D=x, \tau=i\right\}$ is the summation of a Gaussian random variable with mean 0 by a constant. This can be written as follows:

$$
\left\{\Delta \varphi_{1} \mid D=x, \tau=i\right\}=\Delta \varphi_{0}+x .
$$

Relationship (3.10) indicates that the location of the $\mathrm{PH}$ is not affecting the distribution of $\Delta \varphi_{1}$. This is justified by the fact that the $\mathrm{PH}$ happens between 2 symbols 


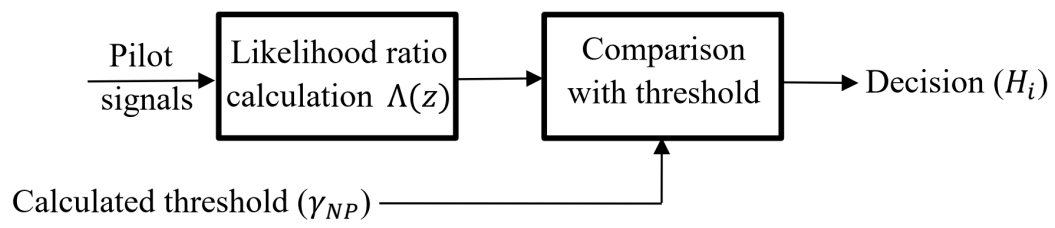

Figure 3.4: The block diagram shows the real-time operations of proposed $\mathrm{PH}$ detection scheme

within the PHPS and its rotational effect is reflected on the next pilot regardless of the PH location. By using (3.5) and (3.10) and dropping $\tau$ from the calculations, we have $\left\{\Delta \varphi_{1} \mid D=x\right\} \sim \mathcal{N}\left(x, \sigma_{P}^{2}\right)$. We can find the PDF of $\Delta \varphi_{1}$ as follows:

$$
\begin{aligned}
f_{\Delta \varphi_{1}}(\delta) & =\int_{x} f_{\Delta \varphi_{1} D}(\delta \mid D=x) \cdot f_{D}(x) \mathrm{d} x \\
& =\frac{1}{d_{M}-d_{m}} \frac{1}{\sqrt{2 \pi \sigma_{P}^{2}}} \int_{x=d_{m}}^{d_{M}} \exp \left\{\frac{|\delta-x|^{2}}{2 \sigma_{P}^{2}}\right\} \mathrm{d} x .
\end{aligned}
$$

The distribution in (3.11) can be written as follows:

$$
f_{\Delta \varphi_{1}}(\delta)=\frac{1}{d_{M}-d_{m}}\left[\mathrm{Q}\left(\frac{d_{m}-\delta}{\sigma_{P}}\right)-\mathrm{Q}\left(\frac{d_{M}-\delta}{\sigma_{P}}\right)\right],
$$

By letting $i=1$ and replacing $f_{\Delta \varphi_{1}}(\delta)$ from (3.12) in (3.6) we can calculate the PDF of $Z_{1}$, i.e., $f_{(Z H)}\left(z \mid H_{1}\right)$. The likelihood function during the $\mathrm{PH}$ operating mode is expressed as follows:

$$
\begin{aligned}
f_{(Z H)}\left(z \mid H_{1}\right) & =\frac{1}{d_{M}-d_{m}}\left[\mathrm{Q}\left(\frac{d_{m}-z}{\sigma_{P}}\right)+\mathrm{Q}\left(\frac{d_{m}+z}{\sigma_{P}}\right)\right. \\
& \left.-\mathrm{Q}\left(\frac{d_{M}-z}{\sigma_{P}}\right)-\mathrm{Q}\left(\frac{d_{M}+z}{\sigma_{P}}\right)\right] u(z) .
\end{aligned}
$$

\subsubsection{PH Detection Using the NP-BHT}

By replacing the likelihood functions in (2.9) and using (2.13) we can find the threshold corresponding to a given probability of FA. Let us show the FA probability with 
$\alpha_{\text {FA }}$. The FA probability depends on the likelihood function during the normal operating mode. By replacing (3.7) in (2.12) we have:

$$
\begin{aligned}
\alpha_{\mathrm{FA}} & =\sqrt{\frac{2}{\pi \sigma_{P}^{2}}} \int_{z \geq \Lambda^{-1}\left(\gamma_{N P}\right)} \exp \left\{\frac{|z|^{2}}{2 \sigma_{P}^{2}}\right\} u(z) \mathrm{d} z \\
& =2 \mathrm{Q}\left(\frac{\Lambda^{-1}\left(\gamma_{N P}\right)}{\sigma_{P}}\right),
\end{aligned}
$$

where we used the fact that $\Lambda(z)$ is a monotonically decreasing function of $z$. In (3.14) $\Lambda^{-1}(y)$ is the inverse of the function $y=\Lambda(x)$. By taking the inverse of $\mathrm{Q}-$ function from both sides of (3.14), we find the threshold which minimizes the $\operatorname{Pr}(\mathrm{DM})$ as follows:

$$
\gamma_{N P}=\Lambda\left(\sigma_{P} \mathrm{Q}^{-1}\left(\frac{\alpha_{\mathrm{FA}}}{2}\right)\right) .
$$

We replace the threshold from (3.15) in (2.14). Considering that the likelihood ratio, $\Lambda(z)$, is a monotonically decreasing function, by taking its inverse from both sides of inequality in (2.14), we have the following equivalent relationship for the designed NP-BHT:

$$
z \underset{H_{0}}{\stackrel{H_{1}}{\gtrless}} \sigma_{P} \mathrm{Q}^{-1}\left(\frac{\alpha_{\mathrm{FA}}}{2}\right) .
$$

The equivalent test in (3.16) is an intuitive explanation of the proposed PH detection scheme, i.e., we compare the variations of the phase offset between two consecutive pilots with a threshold. The threshold is carefully designed such that it minimizes the probability of DM. The minimum $\operatorname{Pr}(\mathrm{DM})$ is calculated by combining (2.13), (3.13) and (3.15) as follows:

$$
\min \operatorname{Pr}(\mathrm{DM})=\int_{z \leq \sigma_{P} \mathrm{Q}^{-1}\left(\frac{\alpha_{\mathrm{FA}}}{2}\right)} f_{Z H}\left(z \mid H_{1}\right) d z
$$


The closed form expression of minimum probability of DM is calculated by solving the integral in (3.17), which yields the following expression:

$$
\begin{aligned}
\min \operatorname{Pr}(\mathrm{DM}) & =1-\frac{\sigma_{P}}{d_{M}-d_{m}}\left[\hat{g}\left(\frac{\gamma_{N P}-d_{m}}{\sigma_{P}}\right)+\hat{g}\left(\frac{\gamma_{N P}+d_{M}}{\sigma_{P}}\right)\right. \\
& \left.-\hat{g}\left(\frac{\gamma_{N P}+d_{m}}{\sigma_{P}}\right)-\hat{g}\left(\frac{\gamma_{N P}-d_{M}}{\sigma_{P}}\right)\right]
\end{aligned}
$$

where $\hat{g}(a) \triangleq a \mathrm{Q}(a)-\frac{1}{\sqrt{2 \pi}} e^{\frac{-a^{2}}{2}}$. The derivation of (3.18) is provided in Appendix A.2.

\subsection{Proposed PH Correction Scheme}

In this section, we assume the proposed algorithm described in Section 3.3 has detected a PH. To mitigate the PH on the affected signals in the PHPS we need to find the precise location of the $\mathrm{PH}$ and its size. The former serves as the starting point where the $\mathrm{PH}$ correction is performed, while the latter determines the value with which we should de-rotate the affected signals. Finding the size of the PH upon detecting it is not a challenging task. The phase offset of the received signals within the PHPS is composed of the Gaussian noise, the LO PN and the PH. The PH only affects the signals after the PH instance. If we find an estimate of the $\mathrm{PN}$ and remove it from the received signals in the PHPS, the phase offset will be dominated by the $\mathrm{PH}$ (from the point where it starts). This happens since the $\mathrm{PH}$ size is much larger than the rotational effect of Gaussian noise and the residual LO PN. Therefore, upon estimating and removing the LO PN from all the signals in the PHPS, the difference between the phase offset of the two pilots in the PHPS gives a rough estimate of $\mathrm{PH}$ size.

To remove the PN in the PHPS, we use the extrapolation of the estimated PN from the previous and the next PSs. The extrapolation of PN from the pre-hit PS yields the PN estimate of the signals before the PH. The extrapolation of the PN from the post-hit PS provides the PN estimates for the signals after the PH. To adopt this approach we need to have the location of the $\mathrm{PH}$. Without the $\mathrm{PH}$ location we can not distinguish the signals, which receive their PN estimate from the pre-hit PS from those which receive their PN estimate from the post-hit PS. To resolve this issue, the 


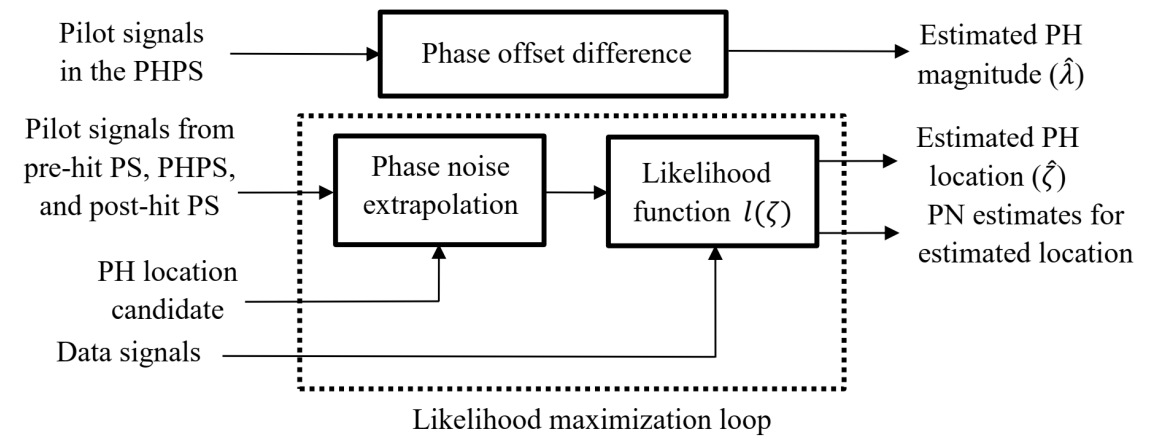

Figure 3.5: Block diagram of proposed $\mathrm{PH}$ correction scheme.

aforementioned PN removal is performed for all the potential locations in the PHPS. To do so, we form a cost function to determine the $\mathrm{PH}$ location. This cost function considers the effect of both the PN and the PH. Fig. 3.5 displays the block diagram of the proposed $\mathrm{PH}$ correction scheme. In the following we describe the derivation of the likelihood function, which is used in the PH location estimation.

\subsubsection{Estimating the PH Location}

To find the location of $\mathrm{PH}$, we use the ML estimation. Let $\xi$ represent the exact location of the PH. The ML estimator finds an estimated location, $\hat{\xi}$, as follows:

$$
\hat{\xi}=\arg \max _{\xi}[l(\xi)],
$$

In (3.19) $l(\xi)$ is the log-likelihood function of the estimation target $\xi$. The location of the PH is within the PHPS; thus, in our estimation we use the information of all the received signals in that PS. The desired log-likelihood function is expressed as follows:

$$
l(\xi)=\log \left[f\left(y_{1}, \ldots, y_{P} \mid \xi\right)\right] .
$$

In (3.20), $f\left(y_{1}, \ldots, y_{P} \mid \xi\right)$ is the joint PDF of all the received signals within the PHPS after removing the LO PN and conditioned on knowing the PH location. The initial received signals before the PN removal follow the model in (3.1). As it was explained, to remove the LO PN when the PH location is known, we propose to use the extrapolation of the PN estimation from the previous and the next PSs. Considering that for 
the high-order modulations the pilot spacing is commonly small (e.g., 50 and lower), we can assume that the LO residual PN on the symbols will be negligible. Under the assumption of perfect LO PN removal using the extrapolation from the adjacent PSs, we can write the de-rotated received signal model as follows:

$$
y_{i}=r_{i} e^{-j \varphi_{\mathrm{PN}, i}}=\left\{\begin{array}{ll}
s_{i}+\hat{n}_{i} & 1 \leq i \leq \xi-1 \\
s_{i} e^{j \lambda(\xi)}+\hat{n}_{i} & \xi \leq i \leq P
\end{array},\right.
$$

where $\lambda(\xi)=\varphi_{1}\left(t_{K+1}\right)-\varphi_{1}\left(t_{K}\right)$ is the estimated $\mathrm{PH}$ size corresponding to the $\mathrm{PH}$ location $\xi$. The parameter $\hat{n}_{i}$ is the circular symmetric Gaussian noise with similar properties to $n_{i}$. The samples of de-rotated received signal, $y_{i}$, are i.i.d. Hence, the joint PDF in (3.20) can be derived as follows:

$$
l(\xi)=\sum_{i=1}^{P} \log \left[f\left(y_{i} \mid \xi\right)\right]=\sum_{i=1}^{P} \log \left[\frac{1}{M} \sum_{n=1}^{M} f\left(y_{i} \mid \xi, s_{n}\right)\right] .
$$

In (3.22), $M$ is the constellation size and we assume all the constellation points are equally likely. From RHS of (3.22) it is observed that $f\left(y_{i} \mid \xi, s_{n}\right)$ is a Gaussian distribution function with the mean $s_{n}$ or $s_{n} e^{j \lambda(\xi)}$ depending on the index $i$. By replacing (3.21) in (3.22) we have:

$$
\begin{aligned}
l(\xi) & =C_{1} \sum_{i=1}^{\xi-1} \log \left[\sum_{n=1}^{M} \exp \left\{-\frac{\left|y_{i}-s_{n}\right|^{2}}{2 \sigma_{n}^{2}}\right\}\right] \\
& +C_{2} \sum_{i=\xi}^{P} \log \left[\sum_{n=1}^{M} \exp \left\{-\frac{\left|y_{i}-s_{n} e^{j \lambda(\xi)}\right|^{2}}{2 \sigma_{n}^{2}}\right\}\right] .
\end{aligned}
$$

The constant $C_{1}$ and $C_{2}$ do not affect the maximization process in (3.19). By replacing (3.23) in (3.19) and solving the optimization problem, the $\mathrm{PH}$ location and the corresponding PH size can be estimated. Unfortunately, evaluation of expression in (3.23) for a large constellation becomes computationally expensive. As an alternative approach, we can simplify the procedure by using the log-sum approximation, $\log \sum_{i} \exp \left(x_{i}\right) \approx \max _{i}\left(x_{i}\right)$. The approximated likelihood function is derived as 
follows:

$$
l(\xi) \approx \frac{C}{2 \sigma_{n}^{2}}\left[\sum_{i=1}^{P}\left|y_{i}-\hat{s}_{i}\right|^{2}\right],
$$

where $C$ is a constant and is ineffective in the estimation of $\mathrm{PH}$ location. The parameter $\hat{s}_{i}$ in (3.24) is calculated as follows:

$$
\hat{s}_{i}=\left\{\begin{array}{ll}
\arg \min _{s_{n}}\left|y_{i}-s_{n}\right|^{2} & 1 \leq i \leq \xi-1 \\
\arg \min _{s_{n}}\left|y_{i}-s_{n} e^{j \lambda(\xi)}\right|^{2} & \xi \leq i \leq P
\end{array},\right.
$$

By combining (3.24) and (3.25) and replacing in (3.19) the location of $\mathrm{PH}$ and the corresponding $\mathrm{PH}$ size can be estimated. In particular, we look for the parameter $\hat{\xi}$, which maximizes (3.24). The maximization is performed by an exhaustive search among all the possible values of $\xi$, i.e., among $P$ locations. Since the $\mathrm{PH}$ is a rare event and the correction scheme is triggered only after the detection of a $\mathrm{PH}$, the use of exhaustive search is justified.

Upon estimating $\hat{\xi}$, and $\hat{\lambda} \triangleq \lambda(\hat{\xi})$, all the received signals in the PHPS are derotated. Let $\hat{y}_{i}$ represent the de-rotated received signals. We use the estimated PH size, $\hat{\lambda}$, and the estimated PN, $\hat{\varphi}_{\mathrm{PN}}$ in the de-rotation. The estimated PN is the result of extrapolation of PN in the pre-hit and post-hit PSs. The received signals after de-rotation are expressed as follows:

$$
\hat{y}_{i}=\left\{\begin{array}{ll}
r_{i} e^{-j \hat{\varphi}_{\mathrm{PN}, i}} & 1 \leq i \leq \hat{\xi}-1 \\
r_{i} e^{-j \hat{\varphi}_{\mathrm{PN}, i}} e^{-j \hat{\lambda}} & \hat{\xi} \leq i \leq P
\end{array},\right.
$$

We denote the residual phase error on the signals after the de-rotation by parameter $\theta$. From (3.26) we have:

$$
\hat{y}_{i}=s_{i} e^{j \theta_{i}}+\tilde{n}_{i}, \quad 1 \leq i \leq P
$$

The de-rotated signals in (3.27) are approximately free of PN and PH. These samples are sent to the LLR calculation module and the rest of the receiver processing will be similar to what was explained in Section 3.2. The described techniques in the 


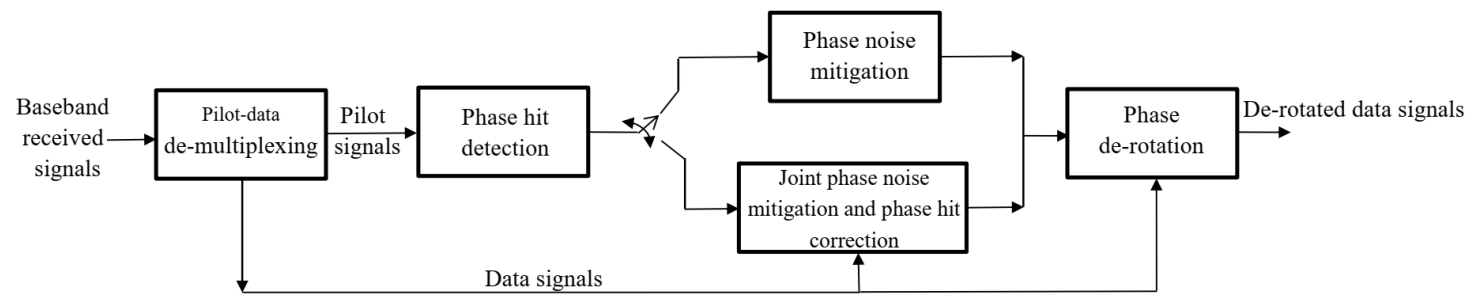

Figure 3.6: Block diagram of proposed $\mathrm{PH}$ mitigation scheme.

proposed PH mitigation scheme is summarized in Fig. 3.6.

\subsection{Simulation Results and Analysis}

The effectiveness of proposed double stage $\mathrm{PH}$ mitigation scheme is investigated in this section. As it was mentioned in Section 3.4, the correction algorithm is triggered after a $\mathrm{PH}$ is detected. The detection algorithm, however, is continuously inspecting the potential occurrence of the PH in every PS. The two algorithms, therefore, operate independently and the only common point between them is that a $\mathrm{PH}$ detection initiates the $\mathrm{PH}$ correction. Likewise, in the following we evaluate the performance of the detection and the correction algorithms separately.

\subsubsection{Performance of PH Detection Scheme}

To evaluate the performance of proposed $\mathrm{PH}$ detection scheme, we consider a QAMLDPC coded system, in which the PSAM scheme is used to mitigate the PN. The constellation is 256-QAM, the symbol rate is $6 \times 10^{6}$ symbols per second and the pilot spacing is 50 . The corner points of the constellation is assumed to be the pilot set. The SNR is defined as $10 \log _{10}\left(1 / \mathcal{E}\left\{|n|^{2}\right\}\right)$ and is assumed to be $26 \mathrm{~dB}$. The LO $\mathrm{PN}$ level at the transmitter and the receiver is $-95 \mathrm{dBc} / \mathrm{Hz}$ at $100 \mathrm{kHz}$ offset from the carrier frequency. Considering that Wiener PN model is used, the PN increment between two consecutive signals follows a Gaussian distribution. The variance of this distribution is calculated using $\sigma_{L}^{2}$ and the symbol interval $T_{s}$. The parameter $\sigma_{L}^{2}$ is calculated using $\sigma_{L}^{2}=4 \pi^{2} f_{0}^{2} \times 10^{\frac{L}{10}}$, which yields $\sigma_{L}^{2} T_{s}=2.08 \times 10^{-5} \mathrm{rad}$. Using the system parameters, the variance which is used in (3.5) and (3.12) is calculated from 


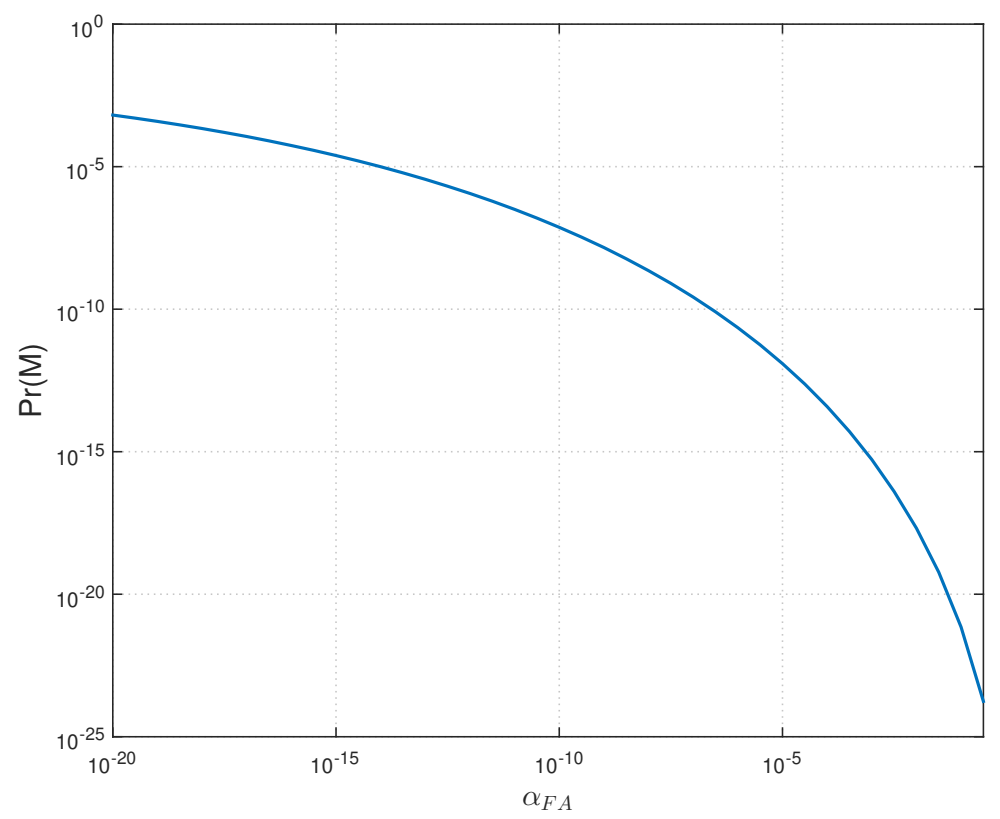

Figure 3.7: The theoretical lower bound on the probability of DM is shown for a wide range of FA probability using (3.15) and (3.18).

its definition in (3.5), which gives $\sigma_{P}^{2}=0.3778$. According to (3.15), the threshold can be found for a given FA probability, $\alpha_{\mathrm{FA}}$. The NP-BHT guarantees that by using this threshold, the probability of DM is minimized. The relationship between $\alpha_{\mathrm{FA}}$ and the achievable $\min \operatorname{Pr}(\mathrm{DM})$ can be found using (3.15) and (3.17). Considering the wide range of the values which the two parameters can take, we show both axes of the curve in the log domain. In Fig. 3.7 the theoretical minimum probability of DM is shown for the different choices of FA probability. For each $\alpha_{\mathrm{FA}}$, initially, the threshold is calculated using (3.15). This threshold is replaced in (3.18) to calculate the minimum probability of DM. We use (3.9) to choose the parameters $d_{m}$ and $d_{M}$. Here $d_{m}=0.53 \mathrm{rad}$ and $d_{M}=5.75 \mathrm{rad}$.

To verify that the designed FA probabilities are capable of minimizing the probability of DM, we simulate the described system. We consider $10^{7} \mathrm{PSs}$, in which every PS experiences a single hit in a random location and with a random size. Considering that $P=50$ the location is chosen uniformly from 1 (first symbol after the pilot) to 50 (the pilot symbol at the end of PHPS). The PH size changes from $d_{m}$ to $d_{M}$. The FA probability is a design parameter and upon choosing it, we find the 


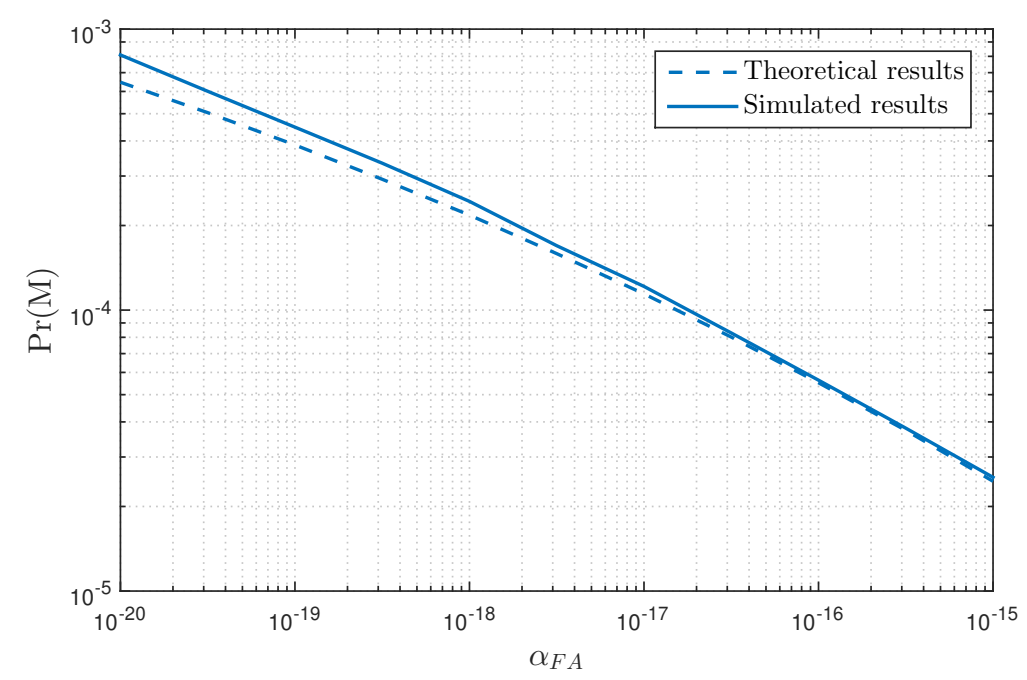

Figure 3.8: The theoretical $\operatorname{Pr}(\mathrm{DM})$ is compared with the same parameter, when it is found using the simulation of $10^{7} \mathrm{PSs}$.

detection threshold using (3.15). Unfortunately, in our simulation we cannot choose a wide range of FA probability similar to one in Fig. 3.7. From Fig. 3.7 it is observed that for the larger $\alpha_{\mathrm{FA}}$ values, probability of DM becomes very small, e.g., when $\alpha_{\mathrm{FA}}>10^{-6}$, we have $\min \operatorname{Pr}(\mathrm{DM})<2 \times 10^{-11}$. This is, in general, desirable from the design standpoint since we want to push $\min \operatorname{Pr}(\mathrm{DM})$ to the lowest possible level. In the simulations, however, we use the Monte-Carlo approach, which requires collection of sufficient samples, e.g., to verify $\operatorname{Pr}(\mathrm{DM})=10^{-10}$ we need to generate at least $10^{12}$ PSs, which is extremely time consuming. We thus limit the simulations to the values of $\alpha_{\mathrm{FA}}$, which result in the relatively higher range of probability of DM. In Fig. 3.8, we verify the left side of the curve in (3.7) through the simulation. This is equivalent to relatively small $\alpha_{\mathrm{FA}}$ and large $\operatorname{Pr}(\mathrm{DM})$ values. The simulated curve of $\operatorname{Pr}(\mathrm{DM})$ in Fig. 3.8 is obtained by dividing the number of failures of detection scheme by the total number of simulated PSs, i.e., $10^{7}$. It is observed that $\operatorname{Pr}(\mathrm{DM})$, which is found from this simulation closely matches the theoretical values. The slightly higher values on the simulation curve compared to the theoretical one is also expected since the theoretical curve is a lower bound on the DM probability and the simulation results (in the best case scenario) can asymptotically achieve those values.

To further verify the detection scheme, we investigate the probability of FA using 
Table 3.1: Probability of FA is found using the simulation of $10^{7}$ PSs. The designed $\alpha_{\mathrm{FA}}$ refers to the chosen value for this parameter. The simulated $\alpha_{\mathrm{FA}}$ is the ratio of the FAs over the total PSs found from the simulation.

\begin{tabular}{|c|c|c|c|c|c|}
\hline designed $\alpha_{\mathrm{FA}}$ & $5 \times 10^{-5}$ & $10^{-4}$ & $10^{-3}$ & $10^{-2}$ & $10^{-1}$ \\
\hline simulated $\alpha_{\mathrm{FA}}$ & $4.91 \times 10^{-5}$ & $9.05 \times 10^{-5}$ & $8.94 \times 10^{-4}$ & $9.6 \times 10^{-3}$ & $9.14 \times 10^{-2}$ \\
\hline
\end{tabular}

the simulation of the PSs in the normal operating mode. Here, all the simulation parameters are similar to those in Fig. 3.8. The only difference is that we set the hit size to 0; thus, all the PSs are working in the normal operating mode. The detection scheme is still inspecting every PS to identify the potential PH events. In this situation, whenever a $\mathrm{PH}$ is detected a FA is given. As opposed to Fig. 3.8 here we consider the smaller values of $\alpha_{\mathrm{FA}}$ since they are considered the achievable FA probabilities when using the simulation. Table. 3.1 displays the values of the FA probability which are chosen as the design parameter and the values of the FA probability which are found by using the simulation. It is observed that the FA rate of the proposed detection scheme is very close to the designed numbers. It is worth noting that in a practical scenario, we are interested in having very small number of FA and DM cases. An FA causes the correction scheme to initiate and thus increases the processing latency of the system. It also can cause system degradation due to the de-rotation of some of the signals in the PS. A DM, on the other hand, will result in a burst of errors in the signals within the PS as it was explained in Section 3.1.

\subsubsection{Performance of PH Correction Scheme}

In this section, we investigate the performance of the correction scheme when a $\mathrm{PH}$ is detected. We assume the detection algorithm has identified a PH event. According to what was explained in Section 3.4, we need to form the likelihood function (the exact one in (3.23) or the approximated one in (3.24)) and find the value which maximizes that function. The size of the $\mathrm{PH}$ is estimated by finding the difference of the phase offset on the pilots in the PHPS. It is worth noting that when forming the approximated joint likelihood function in (3.24), the minimization is carried out by finding the point closest to the de-rotated received signals. This type of minimization 
is used to find the channel LLRs which are fed to the LDPC decoder. Thus, the required module is readily available in most practical systems. We, however, investigate the proposed correction scheme by considering both the exact likelihood function in (3.23) and the approximated likelihood function in (3.24) to evaluate the potential degradation due to use of the approximated expression.

The detected $\mathrm{PH}$ location by applying the correction algorithm is used to derotate the received signals by the angle which is equal to the estimated $\mathrm{PH}$ size. Considering that in almost all the practical systems the FEC is used, a small error in finding the $\mathrm{PH}$ location is tolerable to the system. Note that an error of $a$ positions in estimating the $\mathrm{PH}$ location results in $a$ symbols to stay in error due to the $\mathrm{PH}$ and thus (at most) $a \log _{2} M$ channel LLRs will become inaccurate or invalid. If $a \log _{2} M$ is more than the error correction capability of the FEC, the invalid LLRs can cause the decoder failure. On the other hand, if $a \log _{2} M$ is within the range of the error correction capability of the FEC, the error of $a$ positions in estimating the PH location is acceptable. Choosing $a$ depends on the FEC characteristics and the constellation size but in our simulations, we let $a=2$, which results in the relatively small number of invalid LLRs.

Let random variable $A$ represent the distance between the estimated location of $\mathrm{PH}$ to the exact $\mathrm{PH}$ location and let $F_{A}(a)=\operatorname{Pr}(A \leq a)$ be the cumulative distribution function $(\mathrm{CDF})$ of $A$ at point $a$. For example, $F_{A}(0)$ indicates the probability of finding the exact hit location while $F_{A}(2)$ is the probability of detecting a location within the PHPS, which is not more than 2 symbols away from the exact hit location. This gives that (for $a=2$ ) our correction scheme only fails to mitigate the PH effect on (at most) 2 symbols. Even for a large constellation, e.g., 1024-QAM this is equivalent to have 20 invalid channel LLRs. This is within the error correcting capability of the modern FEC codes of large length, e.g., the LDPC codes used in PtPMRLs. Fig. 3.9 displays the aforementioned CDF when similar system parameters to those in Section 3.5.1 is used. To estimate the CDF, $10^{4}$ PSs are used, and the location and the size of the $\mathrm{PH}$ is randomly selected. The exact likelihood function and the approximated one are both used and the difference between the results is negligible. This is what we observe for all the other simulation scenarios and thus remove the curves corresponding to the exact likelihood function in the rest of the figures in this 


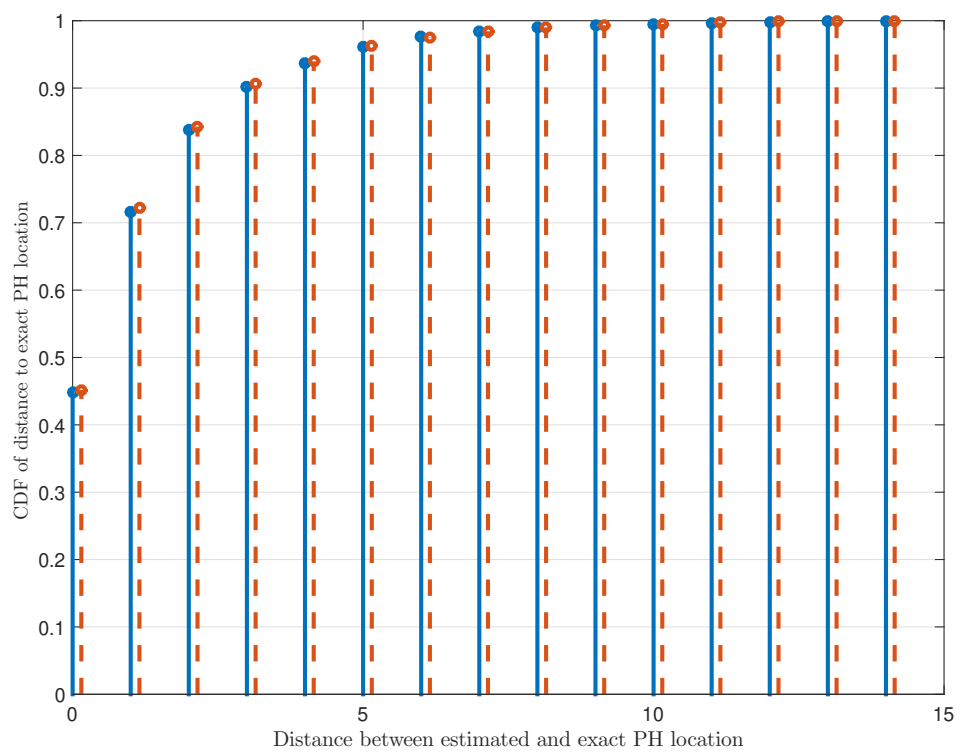

Figure 3.9: $\mathrm{CDF}$ of the distance between the detected location and the actual location of the PH. The number of total simulated PSs is $10^{4}$, the constellation is $256-\mathrm{QAM}$, and the $\mathrm{SNR}$ is $26 \mathrm{~dB}$. The size of the $\mathrm{PH}$ is set to $130^{\circ}$

section. From Fig. 3.9 it is observed that there is $85 \%$ chance that the correction scheme locates the actual PH location or its first 2 neighbors, while the chances of locating a point in a distance less than 5 from the actual $\mathrm{PH}$ location is almost $98 \%$.

To evaluate the effect of $\mathrm{PH}$ characteristics on the performance of proposed correction scheme, we estimate the $\mathrm{CDF}$ under different conditions. In Fig. 3.10, $F_{A}(2)$ is displayed for various PH locations. For a given location within the PHPS, $10^{4} \mathrm{PSs}$ are simulated. The PH size in each location is randomly selected and the probability of locating the $\mathrm{PH}$ in the distance 2 (or less) from the actual PH location is found. To do so, we measure the distance between the detected location and the actual PH location in each simulated PS. The curve in Fig. 3.10 indicates that the CDF value is higher near the edges of the PHPS while the points in between have similar CDF. The values of $F_{A}(2)$ however, are changing in a relatively small range. This verifies that the proposed correction scheme shows a similar performance regardless of the hit location.

As a further step to verify the effectiveness of the proposed correction scheme, we 


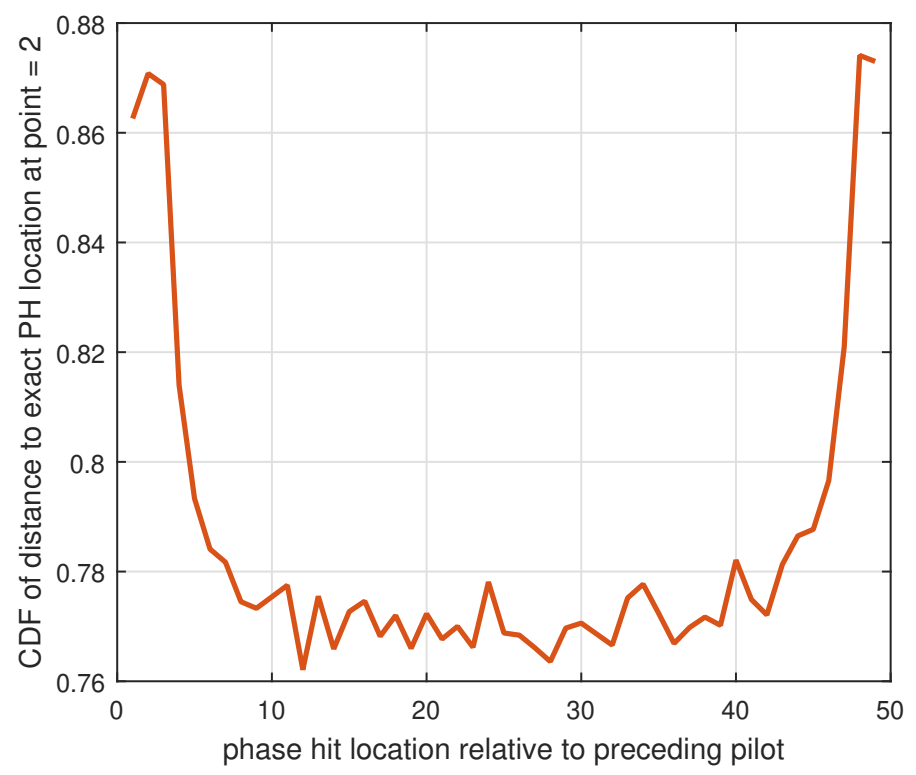

Figure 3.10: Effect of $\mathrm{PH}$ location on the CDF of the distance between the detected location and the actual location of the $\mathrm{PH}$ is shown for $F_{A}(2)$. Pilot spacing is 50, the number of simulated PSs is $10^{4}$, the constellation is 256-QAM, the SNR is $26 \mathrm{~dB}$, and the $\mathrm{PH}$ sizes are randomly and uniformly selected from the interval $[0.5,5.8] \mathrm{rad}$.

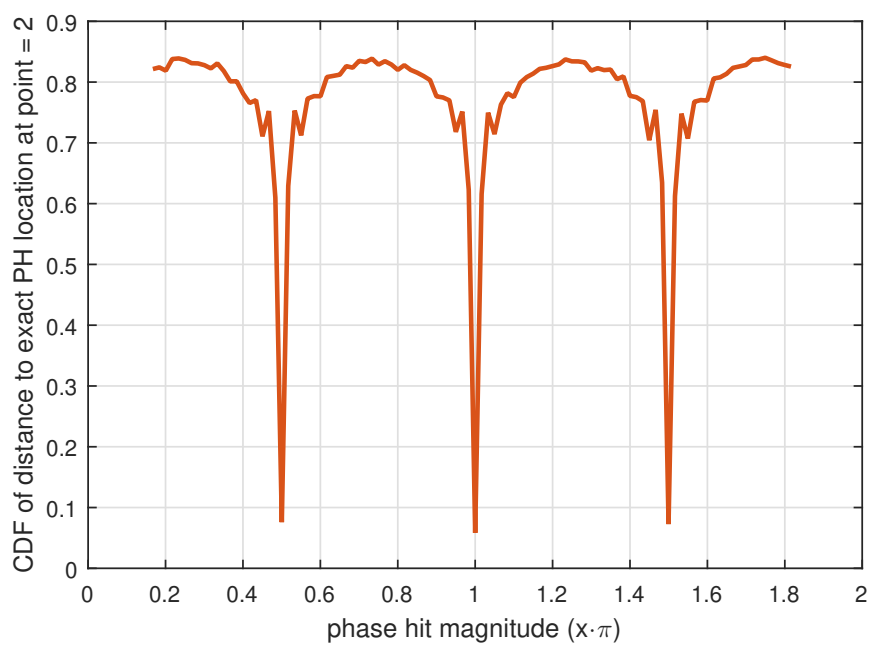

Figure 3.11: Effect of $\mathrm{PH}$ size on the $\mathrm{CDF}$ of the distance between the detected location and the actual location of the $\mathrm{PH}$ is shown for $F_{A}(2)$. Pilot spacing is 50, the number of simulated PSs is $10^{4}$, the constellation is 256-QAM, the $\mathrm{SNR}$ is $26 \mathrm{~dB}$, and the PH location is set to 25

show the CDF function of parameter $A$ when the PH size is kept constant. Here we divide the interval of all the possible PH sizes to 100 discrete points. Every PH size is 
applied in a random location within the PSs. Similar to the previous figures, $10^{4} \mathrm{PSs}$ are simulated for each $\mathrm{PH}$ size and the $\mathrm{CDF}$ at point 2, i.e., $F_{A}(2)$ is shown in Fig. 3.11. It is observed that the correction scheme is detecting the location of the $\mathrm{PH}$ for most of the sizes. The only exception, however, happens when the $\mathrm{PH}$ size is very close to the symmetry angle of the constellation. Note that the square QAM constellations are invariant under the rotation of integer multiples of $\frac{\pi}{2}$. In these situations, i.e., when the PH size is equal to the symmetry angle of the constellation, the cycle slip happens and the ML detection fails to detect the PH location. This deficiency, however, does not pose a severe threat to efficacy of the proposed correction scheme. Note that the $\mathrm{PH}$ size can take any number in a continuous interval of $\left[d_{m}, d_{M}\right]$; thus, the probability of it at any single point tends to 0 . This means that it is highly unlikely for the $\mathrm{PH}$ size to become exactly equal to the symmetry angles of the constellation.

\subsubsection{BER Results}

As a final performance evaluation of the PH mitigation scheme, we show the BER results for a typical high-order coded modulation link. The LDPC code is chosen to be MacKay $(1008,504)$ [62]. We consider 256-QAM with pilot spacing of 32 and 1024-QAM with pilot spacing of 16. Rest of parameters are similar to those in Section 3.5.1. In the PH detection, we set $\alpha_{\mathrm{FA}}=10^{-5}$. We assume one PH per LDPC code block. This scenario is unlikely to happen in practice; nevertheless, this biasing aims at investigating the worst case scenario, and shortening the simulation time. One solution to obtain an unbiased BER result is to introduce a weight function to account for the biasing that is caused by assuming one PH per code block in our simulation. This weight function takes the probability of $\mathrm{PH}$ occurrence into account. Hence, it can transform a biased simulation to an unbiased one. However, such re-weighting is not applied in the following simulations. The reason is twofold: 1) There is no accurate information about the probability of $\mathrm{PH}$ occurrence. Such probability is a function of parameters such as temperature, and it is difficult to estimate or predict, 2) The purpose of BER result comparison in this section is to compare the BER performance of the system with and without applying the proposed $\mathrm{PH}$ mitigation scheme. The biased scenario used in the following merely reduces the simulation time required to 


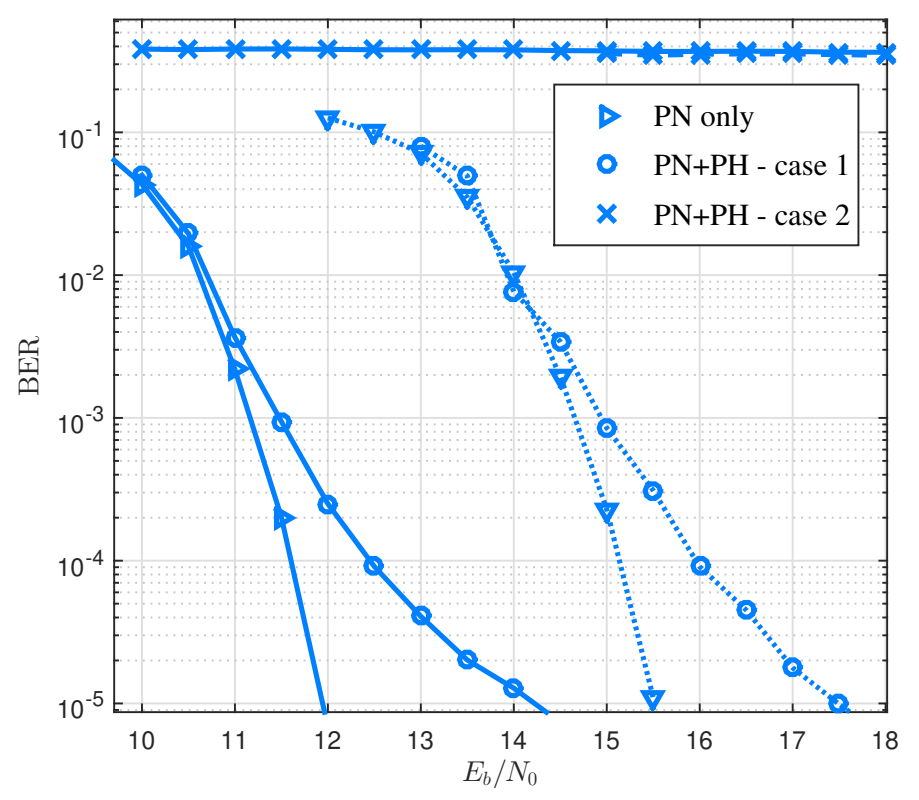

Figure 3.12: BER of 256-QAM (solid line) and 1024-QAM (dashed line) LDPC CMSs are displayed. Case 1: proposed $\mathrm{PH}$ mitigation is applied, Case 2: no $\mathrm{PH}$ mitigation is applied. All the curves are drawn in the presence of $\mathrm{PN}$.

do such comparison but it does not affect its results. We consider the following three scenarios: 1) The system is affected by the PN only, 2) The system is affected by the PN and the PH but no PH mitigation is applied, and 3) Similar to second scenario but this time the proposed double stage PH detection and correction scheme is applied. Fig. 3.12 illustrates the BER results in three aforementioned scenarios. Note that the horizontal axis in Fig. 3.12 is $E_{b} / N_{0}$, i.e., the energy per information bit divided by the noise power spectral density. This value is obtained by taking the effect of pilot overhead and the redundancy of LDPC code into account. The corresponding carrier-to-noise ratios will be $5.53 \mathrm{~dB}$, and $5.96 \mathrm{~dB}$ higher for 256-QAM and 1024QAM constellations, respectively. The curves drawn with solid line correspond to 256-QAM and those with dotted line correspond to 1024-QAM. It is observed that at the absence of $\mathrm{PH}$ correction scheme, the BER is severely deteriorated. In fact if the PH happens frequently (similar to our biased simulation scenario), while no PH mitigation is used, the LDPC decoder almost always fails to converge to the correct codeword and the BER increases to 0.3. For the case of 256-QAM constellation, and by assuming one PH per hour (cf. Section 3.1), the BER in the absence of the 
proposed PH mitigation scheme becomes $0.3 \times \frac{1}{3600 \times 6 \times 10^{6} / 126}=1.75 \times 10^{-9}$, where 126 is the number of symbols per codeword when 256-QAM signalling is used. Hence, if the proposed $\mathrm{PH}$ mitigation scheme is not used, the BER requirement of $10^{-12}$ cannot be met. Similar calculation for the case of 1024-QAM signalling (with an average of 100.8 symbols per codeword) results in a BER of $1.4 \times 10^{-9}$. In contrast, by applying the proposed PH mitigation scheme for SNRs larger than $12 \mathrm{~dB}$ for 256QAM signalling, and $15.7 \mathrm{~dB}$ for 1024-QAM signalling, the BER of the system falls below $10^{-12}$. For instance, at an SNR of $13 \mathrm{~dB}$ with 256-QAM signalling, and one $\mathrm{PH}$ occurrence per hour, the BER of the system is $4 \times 10^{-5} \times \frac{1}{3600 \times 6 \times 10^{6} / 126} \approx 2.33 \times 10^{-13}$. This calculation confirms that by applying the proposed $\mathrm{PH}$ mitigation scheme, the BER of the system will not be limited by the degradation caused by the $\mathrm{PH}$ as it was the case when the proposed PH mitigation scheme was not used.

Furthermore, when the proposed PH mitigation scheme is applied, the BER performance is close to normal operating mode, when we have PN only. The remained gap in the BER curves is attributed to the symmetry angles of the constellation, which upon generating large number of random PH sizes are inevitably generated. On the other hand, in rare cases, the distance between the estimated location of PH and the exact one becomes large, which renders the proposed scheme ineffective. As it was shown in Section. 3.5.2 the probability of such failures are low and the proposed scheme can compensate the $\mathrm{PH}$ impairment effectively in majority of cases.

\subsection{Computational Complexity of Proposed PH Mitigation Scheme}

In Sections 3.3 and 3.4, we described the proposed algorithms for detecting and correcting PH in a PtPMRL. In this section, the computational complexity that each method imposes on the system is investigated. Since the PH is a rare phenomenon, using a computationally complex algorithm is not justified. Fortunately, the proposed $\mathrm{PH}$ detection and $\mathrm{PH}$ correction schemes benefit from low computational burden as it is discussed in the following. 
One significant advantage of the proposed PH detection scheme is the small computational burden that it imposes on the system. In particular, the detection threshold, $\gamma_{N P}$, is calculated using (3.15), which involves evaluating the likelihood ratio at $z=\sigma_{P} \mathrm{Q}^{-1}\left(\frac{\alpha_{\mathrm{FA}}}{2}\right)$. The likelihood ratio is obtained by replacing (3.7), and (3.13) in (2.9). Equation (3.7) is a scaled Gaussian PDF, while (3.13) is the summation of four Q-functions. Hence, the likelihood ratio calculation includes evaluating a Gaussian PDF, a Q-function, and an inverse Q-function. Note that calculating Q-function and inverse Q-function in this equation is commonly done by using look-up tables. In addition to threshold calculation, one needs to measure the phase offset of two consecutive pilots, subtract them and take the absolute value. The phase offset calculation is part of the PSAM PN mitigation. So the values can be reused. Finally, the subtraction and taking absolute value are the operations that can be performed with negligible complexity.

As it was explained in Section 3.3.4, the proposed PH detection scheme relies on pilot symbols that are used in the PSAM scheme to mitigate the PN. Hence, the PH detection scheme does not require any extra overhead in addition to what is used by the PSAM PN mitigation method. Furthermore, our detection scheme is not sensitive to pilot spacing. In the case of using lower order modulations, one may increase the pilot spacing but this change will not affect the $\mathrm{PH}$ detection performance.

With respect to the $\mathrm{PH}$ correction scheme, we consider the case where the approximated problem in (3.24) is solved. This is because the simulation results in Section 3.5.2 show that compared to (3.23), the minimization of (3.24) causes small performance degradation but significant computational complexity reduction. In particular, assessing (3.23) to minimize $l(\xi)$ requires evaluating the argument of the summation in (3.23) MP times for every $\mathrm{PH}$ location candidate, where the argument is an exponential function. In contrast, when the approximated formula in (3.24) is used, only $P$ (degree two) polynomials are evaluated. Furthermore, the minimization in $(3.25)$ can be performed by invoking the geometry of the constellation. This task is commonly performed as part of the LLR calculation. Hence, the same calculation block can be used. 


\section{Chapter 4}

\section{Error Floor Estimation of PAM-based LDPC Coded Modulation Systems}

FEC codes are indispensable in modern digital communication systems, e.g., in the PtPMRLs. Among popular FEC codes are the LDPC ones. These codes enable the system to operate reliably close to the Shannon limit while meeting practical complexity and latency constraints imposed by the system. In addition to favorable performance, LDPC codes also exhibit design flexibility and robustness that renders them suitable in a wide range of applications. For instance, the WiMAX IEEE 802.16e [63], the Advanced Television System Committee [24] and the second generation digital video broadcast standards (DVB-S2) [64] have all adopted LDPC codes. LDPC codes are also preferred in point-to-point backhaul microwave links [8], and have been recently approved by the Third Generation Partnership Project (3GPP) to be the main FEC in upcoming 5G new radios (5G-NR) [65].

To support reliable communication of high data rates over a given bandwidth, LDPC codes are typically combined with high order modulation schemes, e.g., highorder pulse amplitude modulation (PAM) [24]. Combining LDPC codes with high order modulations schemes result in serious challenges to the system design [2224]. One of these challenges pertain to assessing the high SNR performance of the system. In particular, systems using LDPC codes in conjunction with higher-order modulation tend to exhibit an EF. Since the high-order LDPC-CMSs are widely used in the PtPMRLs, it is necessary to investigate the level of the EF in these systems. Traditionally, this investigation would be performed using MC simulations. However, 
for practical systems using LDPC codes, EFs arise at FERs orders of magnitude less than those that can be accurately assessed using such simulations within a reasonable time frame.

The error rate performance of linear block codes, including LDPC ones, can be estimated using a computationally-efficient technique known as importance sampling (IS), see e.g., [27,28]. By invoking the TS structure, this technique can also be used to assess the EF performance of LDPC codes $[25,29]$. To do so, the IS technique induces rare error events more frequently through the use of the analytical expressions of the noise distribution to control the frequency with which error events occur. Approaches for inducing such errors in binary phase shift keying (BPSK) systems were introduced in [25] through an orthogonal transformation of the signalling bases and in [29] through selective biasing of noise components. To elaborate, let us focus on the technique developed in [29] when the all-zero codeword is transmitted. In this case, all the bits are mapped to one of the BPSK symbols, say e.g., s. A key observation in [29] is that decoding fails if the noise components corresponding to the VNs of a TS are sufficiently strong to cause $s$ to be received within the decision region of $-s$ instead of that of $s$. In other words, the TS nodes prevent the message-passing decoder from correcting the erroneous bits, as it would normally do when the VNs do not belong to a TS structure. Using this observation, erroneous decoding events due to a given TS can be triggered by biasing the noise components corresponding to the VNs of this TS. An unbiased estimate of the rate of decoding failure is subsequently obtained by weighting the errors simulated by the biased distribution.

A TS spans a particular subgraph in the Tanner graph that describes the given LDPC code. The structure of such subgraphs can be used to classify various types of TSs, see e.g., [37,40,42,66-68]. Among these structures is the elementary TS (ETS) one [37], which comprises CNs with at most two edges, i.e., with degree less than or equal to 2. In AWGN channels, such ETSs are considered to be the main cause of failure of the message passing algorithm in the high SNR regime (where the EF occurs) [38-40]. For a given LDPC code, we will use the approach proposed in $[40,42]$ to obtain an exhaustive set of all its ETSs.

Based on the premise that the EF of a given LDPC code is an artifact of its underling TS structures, several approaches have evolved to use the IS technique 
and variations thereof to efficiently predict these EFs for various channel conditions and TS structures, see e.g., [29,69-75]. For instance, in [69] an AWGN channel is considered and a method for identifying the error boundary of the decoder for a given TS is proposed. This boundary is subsequently used to evaluate the EF by invoking the IS technique. An approach similar to the one in [69] has been proposed in [74] but for the case in which the channel is Rayleigh fading. Other applications of IS for evaluating the EF of the LDPC codes in AWGN channels are based on shifting the mean of the noise distribution to induce more frequent errors in the TSs without considering a deterministic error boundary, e.g., [29,73,75]. In addition to AWGN channels, this approach is also adopted to evaluate the EF performance of the LDPC codes for other channel models, e.g., the partial response channel [70], and the magnetic recording channel [71]. The application of IS scheme has also been extended to the case of non-binary LDPC codes in [76].

Apart from [72], the majority of existing work in the area of EF estimation based on IS application to TS structures has focused on the case of BPSK signalling. However, future communication systems are likely to rely on coded modulation schemes wherein an FEC, e.g., an LDPC code is combined with higher order modulation schemes. Unfortunately, such LDPC-CMSs with higher order modulation are not amenable to the EF prediction methods developed for BPSK signalling [69, 73, 75].

Towards predicting bounds on the EF of LDPC-CMS, in [72] the method proposed in [25] is extended to a system with $M$-PAM signalling. In this method, bounding the EF relies on using MC simulation to identify the so-called "stable TSs". By construction, such a search is not conclusive: it relies on simulating random events that are not guaranteed to occur over any finite number of simulation trials. This drawback becomes more pronounced as the number of stable TSs in the code becomes larger. In such a case, considerably more block errors must be observed in order for the majority of TSs to be detected. The complexity inherent in the approaches in [25] and [72] is further compounded when the modulation scheme is non-binary. In such a case, assuming that performance is invariant to the choice of the transmitted codeword is invalid. Hence, using the methods in [25] and [72] to bound the EF in the case of non-binary modulation requires individual codewords, or distinct representatives thereof, to be considered, rather than just the all-zero codeword. This approach 
is useful in finding lower bounds on the EF when the number of TSs is relatively low. However, it suffers from a major weakness: this approach is computationally prohibitive when the number of TSs is large. For instance, for a relatively short length-100 LDPC code, the number of TSs can be in the order of hundreds and the number of possible codewords can be in the order of nonillions $\left(10^{30}\right)$ ! In such a case, using the approach in [72] to obtain a tight lower bounds that take into consideration the impact of all TSs that contribute to the EF is practically infeasible.

In this work, we develop a novel EF estimation technique analogous to the technique developed in [29] for BPSK but for higher-order LDPC-CMSs. The new technique is computationally efficient and hence enables the assessment of general LDPCCMSs in the EF region; a task which was practically infeasible using existing techniques, e.g., the one in [72]. To achieve this goal for a given LDPC code, we use the approach in [40] and [42] to identify all the ETSs of a particular size. This approach relies on the graphical characterization of the TSs to find an exhaustive collection of ETSs without invoking any simulations. Hence, this approach offers the potential of alleviating one of the key pitfalls of the method proposed in [72]. However, direct application of the approach in [40] and [42] may result in a prohibitively large number of TSs, resulting in excessive computational burden in estimating the EF especially for large code lengths. To circumvent this difficulty, we propose a categorization scheme whereby the TSs that are likely to have a higher impact on performance are identified. These sets will be referred to as potentially dominant TSs (PDTSs). Subsequently, we define an equivalence class whereby each class corresponds to TSs that map to the same bit position in the constellation. This classification can be readily applied to high-order modulation schemes and subsumes the method developed in [29] for BPSK constellation as a special case. For each equivalence class, we choose a representative PDTS on which we apply an IS framework to obtain the corresponding error rate performance. An estimate of the error rate performance of the entire equivalence class is obtained by scaling the error rate performance of the representative by the cardinality of the equivalence class. Applying this approach to all equivalence classes of a particular class yields an estimate of the error performance of the TSs of that class. An estimate of the overall error rate performance is obtained by adding the contribution of all the considered TS sizes. From this discussion, it can be seen 
that the proposed technique effects substantial computational savings by distilling the TSs in three successive steps. In the first step, the graphical approach in [40] and [42] is used to identify the TSs instead of the MC-based approach used in [72]. In the second step, PDTSs within all the TSs of a particular size are identified using a novel analytical approach. Finally, in the last step members of each PDTS group are categorized into equivalence classes using a generalized version of the approach developed in [29] for BPSK constellations. We notice that the computational burden of extracting and distilling the TSs depends merely on the constellation size and the code structure, but not on the SNR.

Applying the IS method used in $[29,73]$ directly to non-binary signalling scenarios seems difficult. In particular, in non-binary signalling, the LLR of bits within a given symbol are not statistically independent. Hence, to alleviate this difficulty, we modify the method in $[29,73]$ to make it applicable in non-binary signalling. To do so, we replace the biasing of the LLR that underlies the original IS method in $[29,73]$ with the biasing of the received signal. This change not only facilitates the biasing procedure of the IS technique, which otherwise appears to be a daunting task, but also removes the requirement of deriving and evaluating the typically intricate LLR PDF [77]. For Gray labeled constellations, we develop an algorithm that makes use of the constellation geometry to determine a range of mean shift (MS) values that ensures appropriate biasing of the received signals pertaining to a given TS. For a given TS, the range of MS values is a variable that is determined based on the transmitted codeword. This is in contrast with the standard approach in BPSK signalling wherein the all-zero codeword is assumed and the range of MS values is fixed [29,73]. We notice that the computational complexity of determining the MS values and the IS technique depend only on the constellation size and the code structure, but not on the SNR. Numerical simulations show that the proposed framework is capable of estimating the EF of higher-order LDPC-CMSs orders of magnitude faster than MC simulations. In particular, whereas the complexity of the proposed approach for estimating the error rate performance is independent of the operating SNR, that of the MC scheme grows exponentially with the SNR, rendering our approach more attractive in the high SNR regime.

This chapter is organized as follows. In Section 4.1, we describe the IS technique 
and its application in the EF estimation of LDPC codes with BPSK signalling. Section 4.2 contains the main contribution of this work. In particular, in this section, we develop a novel IS technique based on the one in Section 4.1 to estimate the FER of high order LDPC-CMSs with PAM signalling. Furthermore, in this Section, we develop algorithms for efficient extraction of the PDTSs and for identifying the corresponding equivalence classes. Section 4.4 summarizes the steps required to implement the proposed EF estimation scheme and in Section 4.5 we provide numerical results and comparisons.

We use standard notation throughout. Boldface lower and upper case letters are used to represent deterministic and random vectors, respectively. Regular face lower and upper case letters are used to represent deterministic scalars and random variables, respectively. A random variable $X$ will be declared Gaussian with mean $\mu_{X}$ and variance $\sigma_{X}^{2}$ by writing $X \sim \mathcal{N}\left(\mu_{X}, \sigma_{X}^{2}\right)$.

\subsection{Application of MC and IS Schemes for FER Estimation in LDPC Codes}

In this section, we begin by briefly reviewing the application of the IS scheme in evaluating the FER of LDPC codes [28]. We then show how this scheme can be used to estimate the EF in LDPC-coded systems with BPSK modulation [29, 73]. This description will be later used to establish our methodology for higher order modulation in Sections 4.2.

The input of the LDPC decoder is a length- $N$ random vector, $\boldsymbol{L}$, containing the channel LLRs, i.e., the LLR values corresponding to the received bits. We denote the PDF of the channel LLRs by $f_{\boldsymbol{L}}(\boldsymbol{l})$, which depends on the channel model and the constellation symbols to which the transmitted bits are mapped [77]. Practical LDPC decoders use either the sum-product (SP) algorithm or an approximation thereof known as the min-sum algorithm [26]. In the SP algorithm, the channel LLRs are used as a priori information about the received bits being decoded. This information is used to synthesize the LLRs at each VN and CN. These LLRs are iteratively updated following a message-passing fashion; see [26] for details on the SP algorithm. 
At the end of each iteration, the decoder updates the LLRs of all VNs and makes a tentative hard decision on the received bits. Should this decision lead to a binary vector satisfying all the check equations, the decoder is declared to have converged to a valid codeword and decoding stops. If a valid codeword is not found after a prescribed number of decoding attempts, decoding is declared to have failed. We will denote the probability that the decoder fails by

$$
\begin{aligned}
P_{E} & =\int_{\boldsymbol{l} \in \boldsymbol{L}_{E}} f_{\boldsymbol{L}}(\boldsymbol{l}) d \boldsymbol{l} \\
& =\mathcal{E}_{\boldsymbol{L}}\left\{\mathbb{I}_{\boldsymbol{L}_{E}}(\boldsymbol{l})\right\} \\
& =\operatorname{Pr}\left\{\boldsymbol{L} \in \boldsymbol{L}_{E}\right\},
\end{aligned}
$$

where $\boldsymbol{L}_{E}$ denotes the error region containing all $N$-tuple LLRs which cause the decoder to fail, $\mathcal{E}_{X}\{\cdot\}$ denotes the expectation with respect to the random variable $X$, and $\mathbb{I}_{\boldsymbol{L}_{E}}(\boldsymbol{l})$ denotes the indicator function, whereby $\mathbb{I}_{\boldsymbol{L}_{E}}(\boldsymbol{l})=1$ if $\boldsymbol{l} \in \boldsymbol{L}_{E}$ and zero otherwise. To estimates $P_{E}$ two main methods are available, one is based on $\mathrm{MC}$ simulation and the other is based on IS.

The MC simulation estimates $P_{E}$ by approximating the expectation $\mathcal{E}_{\boldsymbol{L}}\left\{\mathbb{I}_{\boldsymbol{L}_{E}}(\boldsymbol{l})\right\}$ using a finite number of trials, $N_{M C}$. In each trial the channel LLRs contained in the random vector $\boldsymbol{L}$ are fed to the decoder and the MC estimate of $P_{E}$, denoted by $P_{E}^{M C}$, is updated depending on whether decoding fails or succeeds. In particular,

$$
P_{E}^{M C}=\frac{1}{N_{M C}} \sum_{i=1}^{N_{M C}} \mathbb{I}_{\boldsymbol{L}_{E}}^{(i)}(\boldsymbol{l})
$$

Estimating $P_{E}$ using MC simulations is computationally intensive. To avoid this cost, an estimation technique based on IS can be used. In this technique, the channel LLRs are chosen from a biased distribution, rather than the original LLR distribution implicitly invoked in the MC-based technique. The biased distribution used in IS controls the probability with which the decoder fails. To do so, let $f_{\boldsymbol{\Lambda}}(\boldsymbol{\lambda})$ be the joint PDF of the channel LLRs when the noise distribution is biased, e.g., by shifting its mean. It is worth noting that shifting the mean is not the only method to bias the noise distribution. Alternative biasing mechanisms exist in the literature, e.g., biasing 
the variance of noise [73]. The choice of the biasing method depends on the underlying application. In this work, we consider an AWGN channel model, and shifting the mean of the noise distribution facilitates the effective biasing of the received signals and the corresponding LLRs. We note that biasing the noise distribution does not affect the error region, $\boldsymbol{L}_{E}$. This is because this region depends solely on the structure of the LDPC code and its decoder, but not on the input thereto. However, biasing the noise distribution results in a biased estimate of $P_{E}$. Hence, to obtain an unbiased estimate, the IS scheme introduces a weighting function $w(\boldsymbol{\lambda})$ in its formulation as follows:

$$
P_{E}=\int_{\boldsymbol{\lambda} \in \boldsymbol{L}_{E}} w(\boldsymbol{\lambda}) f_{\boldsymbol{\Lambda}}(\boldsymbol{\lambda}) d \boldsymbol{\lambda}=\mathcal{E}_{\boldsymbol{\Lambda}}\left\{w(\boldsymbol{\lambda}) \mathbb{I}_{\boldsymbol{L}_{E}}(\boldsymbol{\lambda})\right\}
$$

The LLRs can be readily expressed in terms of the received signals, which we denote by $\boldsymbol{R}$. In particular, each entry of the (random) received vector $\boldsymbol{R}$ yields $m$ LLRs, The $(k-1) m+l$-th entry of $\boldsymbol{\Lambda}$, which corresponds to the $l$-th LLR of the $k$-th received signal, $\boldsymbol{R}_{k}$, can be approximated by [34]:

$\boldsymbol{\Lambda}_{(k-1) m+l} \approx \frac{1}{2 \sigma_{n}^{2}}\left\{\min _{s \in \mathcal{A}_{-l}}\left|\boldsymbol{R}_{k}-s\right|^{2}-\min _{s \in \mathcal{A}_{+l}}\left|\boldsymbol{R}_{k}-s\right|^{2}\right\}, \quad l \in\{1, \ldots, m\}, k \in\left\{1, \ldots, N_{s}\right\}$

where $\boldsymbol{R}_{k}$ is the $k$-th entry of the random vector $\boldsymbol{R}$ and $\mathcal{A}_{+l}$ is the set of constellation symbols with $b_{l}=0$ and $\mathcal{A}_{-l}$ is the set of constellation symbols with $b_{l}=1$; both sets have identical cardinalities. Using this observation, it can be seen that the integration in (4.3) can be expressed in terms of the joint PDF of $\boldsymbol{R}, f_{\boldsymbol{R}}(\boldsymbol{r})$. This will be useful in deriving the IS scheme for the case of PAM signalling in Section 4.2. However, for BPSK signalling is suffices to use the expression in (4.3). From (4.3), it can be seen that, to ensure that $P_{E}$ is identical to the $P_{E}$ expression in (4.1), we must have

$$
w(\boldsymbol{\lambda})=\frac{f_{\boldsymbol{L}}(\boldsymbol{\lambda})}{f_{\boldsymbol{\Lambda}}(\boldsymbol{\lambda})}
$$

To obtain an estimate of $\mathcal{E}_{\boldsymbol{\Lambda}}\left\{w(\boldsymbol{\lambda}) \mathbb{I}_{\boldsymbol{L}_{E}}(\boldsymbol{\lambda})\right\}$, the IS algorithm performs $N_{I S}$ trials, which yield an approximate estimate of $P_{E}$, which we denote by

$$
P_{E}^{I S}=\frac{1}{N_{I S}} \sum_{i=1}^{N_{I S}} w^{(i)}(\boldsymbol{\lambda}) \mathbb{I}_{\boldsymbol{L}_{E}}^{(i)}(\boldsymbol{\lambda}) .
$$


Since $\mathcal{E}\left\{P_{E}^{I S}\right\}=P_{E}$, the IS estimator is unbiased. For the case of PAM signalling, we will find it convenient to use an analogous derivation based on the received signals $\boldsymbol{R}$ rather than the respective LLRs, $\Lambda$.

\subsubsection{Comparison Between MC and IS Schemes}

We now compare the reliability and computation efficiency of the MC and IS schemes.

\section{Reliability Comparison}

To compare the reliability of the MC and IS estimators, we consider the variance of normalized estimation error [73]. In particular, for the MC scheme, the variance of the normalized error of the estimator in (4.2) is

$$
\begin{aligned}
\gamma_{M C}=\frac{\operatorname{var}\left\{P_{E}^{M C}\right\}}{P_{E}^{2}} & =\frac{1}{P_{E}^{2} N_{M C}^{2}} \sum_{i=1}^{N_{M C}} \operatorname{var}\left\{\mathbb{I}_{\boldsymbol{L}_{E}}^{(i)}(\boldsymbol{l})\right\} \\
& =\frac{1}{P_{E}^{2} N_{M C}^{2}} \sum_{i=1}^{N_{M C}}\left(P_{E}-P_{E}^{2}\right) \\
& =\frac{1}{N_{M C}}\left(\frac{1}{P_{E}}-1\right)
\end{aligned}
$$

where $\operatorname{var}\{\cdot\}$ denotes statistical variance and, to arrive at (4.7), we used the fact that $\operatorname{var}\left\{\mathbb{I}_{\boldsymbol{L}_{E}}^{(i)}(\boldsymbol{l})\right\}=P_{E}-P_{E}^{2}$.

Analogous to the MC scheme, for the IS scheme, the variance of the normalized error of the estimator in (4.6) can be readily seen to be given by

$$
\gamma_{I S}=\frac{\operatorname{var}\left\{P_{E}^{I S}\right\}}{P_{E}^{2}}=\frac{1}{N_{I S}}\left(\frac{\mathcal{E}_{\boldsymbol{\Lambda}}\left\{\left(w^{(i)}(\boldsymbol{\lambda}) \mathbb{I}_{\boldsymbol{L}_{E}}^{(i)}(\boldsymbol{\lambda})\right)^{2}\right\}}{P_{E}^{2}}-1\right)
$$

To calculate $\gamma_{\mathrm{MC}}$ and $\gamma_{\mathrm{IS}}$, we note that neither $P_{E}$ nor $\mathcal{E}_{\boldsymbol{\Lambda}}\left\{\left(w^{(i)}(\boldsymbol{\lambda}) \mathbb{I}_{\boldsymbol{L}_{E}}^{(i)}(\boldsymbol{\lambda})\right)^{2}\right\}$ is known and hence must be estimated. An estimate of $P_{E}$ can be obtained from (4.2) and (4.6) for the MC and the IS estimators, respectively, while an estimate of $\mathcal{E}_{\boldsymbol{\Lambda}}\left\{\left(w^{(i)}(\boldsymbol{\lambda}) \mathbb{I}_{\boldsymbol{L}_{E}}^{(i)}(\boldsymbol{\lambda})\right)^{2}\right\}$ can be obtained as $\frac{1}{N_{I S}} \sum_{i=1}^{N_{I S}}\left(w^{(i)}(\boldsymbol{\lambda}) \mathbb{I}_{\boldsymbol{L}_{E}}^{(i)}(\boldsymbol{\lambda})\right)^{2}$. In the forthcoming discussions we will use $\gamma_{M C}$ and $\gamma_{I S}$ with estimated $P_{E}$ and 
$\mathcal{E}_{\boldsymbol{\Lambda}}\left\{\left(w^{(i)}(\boldsymbol{\lambda}) \mathbb{I}_{\boldsymbol{L}_{E}}^{(i)}(\boldsymbol{\lambda})\right)^{2}\right\}$ to compare the reliability of the MC and IS schemes.

\section{A Comparison of Computational Efficiency}

To compare the computational efficiency of the MC and IS schemes, we consider the number of trials required by each scheme to achieve the same reliability, i.e., $\gamma_{M C}=\gamma_{I S}=\alpha$. We denote these numbers by $N_{M C}(\alpha)$ and $N_{I S}(\alpha)$ for the MC and IS schemes, respectively. Using these numbers, the computational gain of IS scheme over the MC scheme can be expressed as

$$
\eta_{\alpha}=\frac{N_{M C}(\alpha)}{N_{I S}(\alpha)}
$$

The simulation results reported in Section 4.5 illustrate that $\eta_{\alpha}$ is significantly greater than one, which is indicative of the computational advantage of the IS estimator over its MC counterpart.

\subsubsection{Using IS for EF Estimation of LDPC Codes With BPSK Signalling}

In this section, we describe the application of the IS scheme in estimating the EF of LDPC codes [73]. This approach, however, is limited to the BPSK modulated systems. Applying this technique to non-binary, e.g., PAM, signalling requires reformulation of the IS framework and the underlying biasing mechanism. We will undertake this task in Sections 4.2 below. Towards estimating the EF of a particular LDPC code, we will use the assumption in $[38,73,78]$ whereby, for a given class of $(a, b)$ ETSs, the error rate of the decoder is identical for all isomorphic TSs, i.e., TSs that can be bijectively mapped to each other, for instance by relabelling the CNs and VNs.

Consider a BPSK modulated LDPC coded system. The $k$-th bit of a length $N$ codeword, denoted by $c_{k}$, is mapped to the $k$-th transmitted constellation point, denoted by $s_{k}$. Here, we assume the mapping is expressed as $s_{k}=1-2 c_{k}, k \in$ $\{1, \ldots, N\}$. The AWGN channel model is assumed and the samples of noise, $W$,

are denoted by $\left\{\mathrm{w}_{k}\right\}_{k=1}^{N}$, where $\sigma_{W}^{2}=N_{0} / 2$. Hence, the $k$-th received signal can be expressed as $r_{k}=s_{k}+\mathrm{w}_{k}$ and the LLR of the $k$-th received bit, denoted by the 
random variable $L_{k}$, is given by $L_{k}=\frac{4}{N_{0}} r_{k}$ [77]. For a typical LDPC code, the LLRs of the received bits of a given codeword can be assumed independent and their joint PDF is $f_{\boldsymbol{L}}(\boldsymbol{l})=\prod_{k=1}^{N} f_{L_{k}}\left(l_{k}\right)$, where $f_{L_{k}}\left(l_{k}\right)$ is the PDF of the $k$-th LLR. Using the aforementioned expression of the LLRs, it can be readily seen that $L_{k} \sim \mathcal{N}\left(\frac{4}{N_{0}} s_{k}, \frac{8}{N_{0}}\right)$. Since in BPSK modulation, the 0 and 1 bits have identical protection levels, the zero codeword is assumed to be transmitted without loss of generality. In this case, we have $s_{k}=1, k \in\{1, \ldots, N\}$.

To estimate the error rate induced by an $(a, b) \mathrm{TS}, \mathrm{T}_{(a, b)}$, the IS scheme biases the LLR vector to prompt the decoder to be trapped in $\mathrm{T}_{(a, b)}$. Let $\zeta_{\mathrm{T}_{(a, b)}}$ be an ordered set containing the indices of the symbols to which the bits in $\mathrm{T}_{(a, b)}$ are mapped. We note that in BPSK signalling, each bit is mapped to one symbol and hence in this case $\zeta_{\mathrm{T}_{(a, b)}}$ contains the labels of the $\mathrm{VNs}$ in $\mathrm{T}_{(a, b)}$. Later, we will consider non-binary signalling, in which case the mapping between the entries in $\zeta_{\mathbf{T}_{(a, b)}}$ and those in $\mathrm{T}_{(a, b)}$ depends on the size of the constellation. In BPSK signalling each received signal corresponds to one channel LLR; thus, the set $\zeta_{\mathrm{T}_{(a, b)}}$ contains the indices of the LLRs that the IS scheme ought to bias. This biasing is performed by shifting the mean of additive noise samples, $\mathrm{w}_{k}, k \in \zeta_{\mathrm{T}_{(a, b)}}$, to ensure that the sign of the respective LLRs are reversed with high probability. We will refer to this scheme as mean shift IS (MSIS) [73]. We will now review the MS-IS scheme presented in [73] for estimating the EF of BPSK modulated LDPC codes. We begin by estimating the error rate due to $\mathrm{T}_{(a, b)}$. Subsequently, we will estimate the overall FER by accumulating the error rate contributions of all TSs.

Let $\mathcal{D} \mathcal{F}$ denote the event that the decoder fails to converge to a valid codeword and let $\operatorname{Pr}\left(\mathcal{D} \mathcal{F}, \mathrm{T}_{(a, b)}\right)$ be the probability that the $\mathcal{D} \mathcal{F}$ event occurs due to the decoder being trapped in $\mathrm{T}_{(a, b)}$. In the forthcoming discussion, we will estimate this probability using the general expression in (4.6).

To obtain an estimate of $\operatorname{Pr}\left(\mathcal{D F}, \mathrm{T}_{(a, b)}\right)$, the IS scheme biases the mean of the noise samples indexed by the entries of $\zeta_{\mathbf{T}_{(a, b)}}$, effectively rendering the corresponding received signal closer to the constellation symbol with the opposite polarity. In particular, let $\mu_{k}$ be the MS of the noise sample $\mathrm{w}_{k}$. For convenience, in [73] the same MS value, $\mu_{0}$, is used for all the noise samples. Since the all-zero codeword is assumed to be transmitted, $s_{k}=1$ for all $k$ and hence the IS scheme uses $\mu_{0}<0$. 
Using this MS, the received biased signals can be expressed as $r_{k}=1+\mu_{0}+\mathrm{w}_{k}$. Subsequently, the respective biased LLRs will be the samples of the random variable $\Lambda_{k}$, where $\Lambda_{k} \sim \mathcal{N}\left(\frac{4}{N_{0}}\left(1+\mu_{0}\right), \frac{8}{N_{0}}\right), k \in \zeta_{\mathrm{T}_{(a, b)}}$, while the distribution of rest of the LLRs remains unchanged. Hence, for $k \in\{1, \ldots, N\}$, we have

$$
\Lambda_{k}= \begin{cases}L_{k} & k \notin \zeta_{\mathrm{T}_{(a, b)}}, \\ L_{k}+\frac{4 \mu_{0}}{N_{0}} & k \in \zeta_{\mathrm{T}_{(a, b)}} .\end{cases}
$$

Samples of this $\Lambda_{k}$ will be the elements of the vector $\boldsymbol{\lambda}$ used to calculate the IS weight function in (4.5). To estimate $\operatorname{Pr}\left(\mathcal{D} \mathcal{F}, \mathrm{T}_{(a, b)}\right)$, we use (4.6) with the weight function computed as follows. Using (4.11), the joint PDF of the $N$ LLRs after biasing can be derived as

$$
f_{\boldsymbol{\Lambda}}(\boldsymbol{\lambda})=\left(\prod_{k \in \zeta_{\mathrm{T}}(a, b)} f_{L_{k}}\left(\lambda_{k}-\frac{4 \mu_{0}}{N_{0}}\right)\right)\left(\prod_{k \in\{1, \ldots, N\} \backslash \zeta_{\mathrm{T}}(a, b)} f_{L_{k}}\left(\lambda_{k}\right)\right) .
$$

Substituting from (4.12) in (4.5) yields

$$
w(\boldsymbol{\lambda})=\prod_{k \in \zeta_{(}(a, b)} \frac{f_{L_{k}}\left(\lambda_{k}\right)}{f_{L_{k}}\left(\lambda_{k}-\frac{4 \mu_{0}}{N_{0}}\right)},
$$

where $\lambda_{k}$ is a sample from the biased distribution $\Lambda_{k}$ with the value of $\mu_{0}<0$ appropriately chosen. (Numerical experiments in [73] suggest $\mu_{0} \in[-2,-1]$.) By using (4.13) in (4.6), the probability $\operatorname{Pr}\left(\mathcal{D F}, \mathrm{T}_{(a, b)}\right)$ can be readily estimated over $N_{I S}$ trials.

To estimate the FER of the code in the EF region all the ETSs of a given size should be considered. Let $\mathcal{T}_{(a, b)}$ be the set of all $(a, b)$ ETSs. Such ETSs can be partitioned into disjoint subsets of isomorphic TSs. Let the $j$-th subset of such TSs be denoted by $\mathcal{T}_{(a, b)}^{j}$ and let the total number of such subsets be $J_{a b}$. Hence, $\sqcup_{j=1}^{J_{a b}} \mathcal{T}_{(a, b)}^{j}=$ $\mathcal{T}_{(a, b)}$. Based on the premise that the FER in the EF region is mainly due to the decoder is being trapped in the ETSs, the FER in this region can be approximated 
as follows [26]:

$$
\operatorname{Pr}(\mathcal{D} \mathcal{F}) \approx \sum_{(a, b) \in \mathcal{B}} \operatorname{Pr}\left(\mathcal{D F}, \mathcal{T}_{(a, b)}\right)=\sum_{(a, b) \in \mathcal{B}} \sum_{j=1}^{J_{a b}} \operatorname{Pr}\left(\mathcal{D} \mathcal{F}, \mathcal{T}_{(a, b)}^{j}\right)
$$

where $\operatorname{Pr}\left(\mathcal{D} \mathcal{F}, \mathcal{T}_{(a, b)}\right)$ and $\operatorname{Pr}\left(\mathcal{D} \mathcal{F}, \mathcal{T}_{(a, b)}^{j}\right)$ are the probabilities that the decoder fails because of being trapped in $\mathcal{T}_{(a, b)}$ and $\mathcal{T}_{(a, b)}^{j}$, respectively. The set $\mathcal{B} \triangleq$ $\left\{(a, b) \mid a, b \in \mathbb{N}, \mathbb{I}(a, b)=1, a \leq a_{\max }, b \leq b_{\max }\right\}$, where $\mathbb{I}(a, b)=1$, if the class $(a, b)$ is considered in the EF estimation and $\mathbb{I}(a, b)=0$, otherwise. This set contains the index pairs that characterize all the TSs considered in estimating the EF of the code. Note that choosing the TS classes in $\mathcal{B}$ depends on the LDPC code. In this work, this set is determined by using MC simulation. In particular, $a_{\max }$ and $b_{\max }$ are set to the largest $a$ and $b$ values that are observed in the early EF stage, respectively. It is worth mentioning that the error contributions of smaller ETSs, i.e., with smaller $a$ and $b$, tend to dominate the EF as the SNR increases [79]. This observation will be confirmed in Section 4.5. In such a case, increasing $a_{\max }$ increases estimation complexity but without making substantial improvement in the estimation accuracy.

Since the ETS entries in $\mathcal{T}_{(a, b)}^{j}$ are isomorphic, the corresponding error rates can be assumed to be identical $[38,73,78]$ and $\operatorname{Pr}(\mathcal{D F})$ in $(4.14)$ can be approximated by

$$
\operatorname{Pr}(\mathcal{D} \mathcal{F}) \approx \sum_{(a, b) \in \mathcal{B}} \sum_{j=1}^{J_{a b}}\left|\mathcal{T}_{(a, b)}^{j}\right| \operatorname{Pr}\left(\mathcal{D F}, \mathrm{\top}_{(a, b)}^{j}\right)
$$

where $\left|\mathcal{T}_{(a, b)}^{j}\right|$ is the cardinality of the set $\mathcal{T}_{(a, b)}^{j}$, and $\mathrm{T}_{(a, b)}^{j}$ is an arbitrary ETS in that set. To use MS-IS to estimate $\operatorname{Pr}\left(\mathcal{D F}, \mathrm{T}_{(a, b)}^{j}\right)$, we invoke (4.6) with (4.13) in the IS scheme described hereinabove.

\subsection{EF Estimation of LDPC Codes with PAM sig- nalling}

In this section, we describe our proposed method to estimate the EF of LDPC-CMSs with PAM signalling. We begin by highlighting the main difficulties encountered in 
estimating the EF in these systems when the underlying signalling is non-binary [72]. To circumvent these difficulties, we develop a novel MS-IS scheme that exploits the relationship between the constellation point closest to the received signal and the corresponding channel LLR values. The proposed MS-IS scheme takes the ETSs of the given LDPC code as its input and yields an estimate of the EF of that code. For practical codes, the number of ETSs can be computationally prohibitive. Hence, to reduce the number of ETS candidates, we note that, in contrast with binary signalling, in the non-binary case, the bits are not equally protected, giving rise to the so-called unequal error protection property. In fact, the protection of each bit depends on its BL in the symbol and the location of the symbol in the constellation. Using this observation, we develop a novel technique for extracting the PDTSs of the given LDPC code. The philosophy of this techniques is to invoke the structure and binary labeling of the constellation to rank the TSs based on the likelihood that they cause the decoding procedure to fail. In particular, in the case of non-binary constellation, multiplying $\operatorname{Pr}\left(\mathcal{D} \mathcal{F}, \mathrm{T}_{(a, b)}^{j}\right)$ by $\left|\mathcal{T}_{(a, b)}^{j}\right|$ as in (4.14) is not valid. This is because in this case, TSs that are isomorphic may have different protection levels from the constellation thereby yielding different error rates. Our numerical results show that the joint application of the two algorithms (PDTS extraction, and MS-IS) results in computationally efficient estimation of the EF of LDPC-CMSs with PAM signalling.

\subsubsection{Considerations for Evolving From BPSK to PAM Sig- nalling}

In this section, we will highlight the key differences between the estimation of EFs in LDPC-CMSs with underlying BPSK and PAM signalling. These differences will be used to infer guidelines that will be instrumental in developing a computationally efficient technique for estimating the EF for the case of PAM signalling.

\section{Effect of BL on Error Protection}

Unlike the case with BPSK signalling, in a PAM-based system the coded bits do not receive equal protection from the constellation. For instance, referring to Fig. 4.1, we 
CHAPTER 4. ERROR FLOOR ESTIMATION OF PAM-BASED LDPC-CMS 63

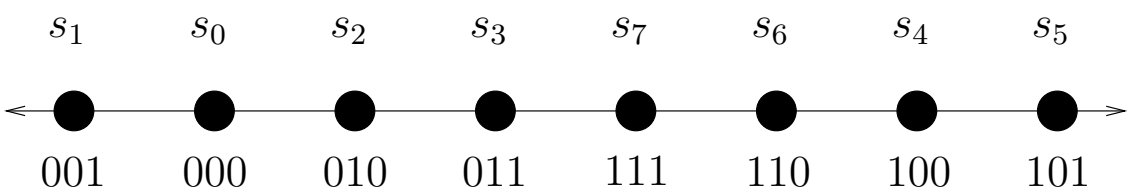

Figure 4.1: A Gray mapped 8-PAM constellation.

note that the first bit of $s_{6}$ is more protected than the second bit. This is because, a noise sample that places the received signal corresponding to $s_{6}$ in the vicinity of $s_{4}$ will result in an LLR with the same sign for the first bit, but with a reversed sign for the second one. An analogous argument applies to the first and third bits in $s_{6}$ and $s_{7}$, respectively. This observation implies that, in contrast with the case of BPSK signalling, in the PAM case, the error rate generated by two isomorphic TSs with the same size are not necessarily equal, since the indices of the VNs in such TSs may correspond to different BLs and hence different error protection levels.

Example 2 For the MacKay $(1008,504)$ LDPC code [62], the (4,2) TS class is known to be dominant [40]. Two such TSs are $T_{1}$ with $V N s\{139,194,521,989\}$ and $T_{2}$ with $V N s\{396,467,548,570\}$, where $T_{1}$ and $T_{2}$ are isomorphic [40]. When this code is used in an LDPC coded modulation system with 32-PAM signalling, the BLs in the constellation symbols corresponding to these VNs are given by the ordered sets $\pi_{T_{1}}=\{4,4,1,4\}$ and $\pi_{T_{2}}=\{1,2,3,5\}$, respectively. The fact that $\pi_{T_{1}} \neq \pi_{T_{2}}$ implies that the error rates incurred by $T_{1}$ and $T_{2}$ are different. In fact, it is expected that $T_{1}$ is more dominant than $T_{2}$ since three of its VNs correspond to bits located at a relatively weak position of $B L=4$, in contrast with only one $V N$ of $T_{2}$ that corresponds to the weak least significant bits (LSB) position of $B L=5$.

This example highlights the fact that BL sets with non-identical elements do not necessarily have the same contribution to the EF even though they belong to isomorphic TSs. Furthermore, the ordering of the elements in the BL sets plays a role in the error contributions. The following example illustrates this effect.

Example 3 Consider the isomorphic TSs: $T_{1}=\{441,478,586,843\}$, and $T_{2}=$ $\{449,726,847,858\}$ from the class $(4,2)$ of the MacKay $(1008,504)$ LDPC code when 16-PAM signalling is used. The corresponding $B L$ sets are $\pi_{T_{1}}=\{1,2,2,3\}$, and 


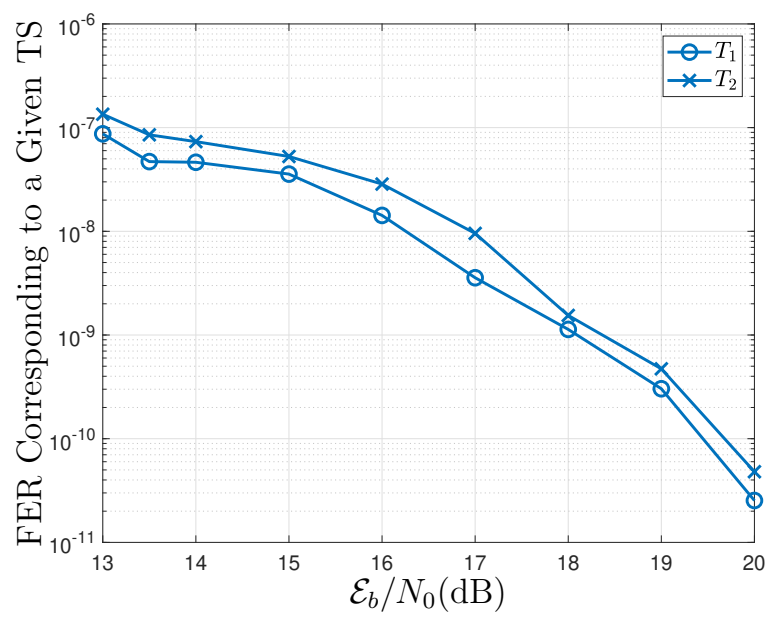

Figure 4.2: Error rate of TSs $T_{1}$ and $T_{2}$ in Example 3.

$\pi_{T_{2}}=\{1,2,3,2\}$, respectively. The elements of $\pi_{T_{1}}$ and $\pi_{T_{1}}$ are identical, but the ordering of the elements in the two sets are different. In Fig. 4.2, the error rates of $T_{1}$ and $T_{2}$ are estimated using the proposed $M S$-IS scheme. It is observed that the error rates of these two isomorphic TSs are different. This difference is attributed to the non-identical ordering of the $B L$ sets corresponding to $T_{1}$ and $T_{2}$.

Since the TSs with BL sets of different order may have non-identical error rates (cf. Example. 3), the order of the BL sets must be included in the categorization of the TSs. An analogous observation has been made for the case of BPSK in [80].

\section{Effect of Noise Biasing on Channel LLRs}

In the BPSK scenario, the derivation of the joint PDF of the LLRs is straightforward since every symbol carries only one bit and the LLR of one bit is assumed to be independent of other bits. In contrast, in the PAM scenario, there are $m=\log _{2} M$ bits mapped to each symbol. In this case, the $m$ LLRs corresponding to each received symbol are dependent [77], and this dependency complicates the derivation of the joint PDF of the LLRs, and renders the biasing of the LLRs as suggested in Section 4.1.2 a formidable task. To circumvent this difficulty, we note that, notwithstanding the complications arising from this dependency, the received signals (conditioned on the transmitted symbol) are statistically independent of each other, and each of these 
signals can be one-to-one mapped to a corresponding set of $m$ LLRs, cf. (4.4). This observation is key to the development of an MS-IS scheme that is appropriate to use in the case of PAM signalling.

To apply the MS-IS scheme, the sign of the LLR of the $l$-th bit if this bit corresponds to a VN of the TS must be reversed; if a bit is not connected to a $\mathrm{VN}$ of the TS, its LLR sign must not be reversed. Doing so directly using the joint PDF of the LLRs in computationally difficult. However, from (4.4) it can be seen that sign reversal of the LLRs of the $l$-th bit can be induced by biasing the received signal by shifting it to lie in the proximity of the closest constellation point in which only the $l$-th bit is reversed. For example, a received signal corresponding to $s_{6}$ in Fig. 4.1 typically lies in the proximity of this symbol in the EF (high SNR) region. Reversing the LLR value of the LSB can be achieved by shifting the received signal to the proximity of $s_{7}$. Shifting the received signal to the proximity of $s_{1}, s_{3}$, or $s_{5}$ will reverse not only the LLR of the LSB, but also the LLRs of other bits, which is not desirable. In Section 4.3, we will elaborate on the appropriate biasing of the received signal to induce sign reversal on bits corresponding to VNs in the TS under consideration.

\section{Effect of Transmitted Codeword}

In the previous section, we showed that the error contribution of isomorphic TSs may not be identical if their BL sets are not identical. In this section, we will show that the error contribution of a given TS depends on the particular codewords being transmitted. To do so, we note that the estimation of the EF in LDPC coded systems which use BPSK signalling is facilitated by assuming that the all-zero codeword is always transmitted $[27,29,73-75]$. In particular, fixing the transmitted codeword in each trial implies that MS values are constant, that is, one MS value is used for all the trials used to estimate the error rate contribution of a given TS. In contrast with BPSK modulation, in the case of PAM signalling, the transmitted symbols and the associated codewords are not equally protected by the constellation. Hence, the MS value must be adjusted for each transmitted codeword. The following example

illustrates the effect of the transmitted codeword on the protection offered by the constellation for the VNs of a given TS. 
Example 4 Consider the TS $T_{2}$ in Example 2 when 8-PAM is used. In this case, the set of BLs is given by $\pi_{T_{2}}=\{3,2,2,3\}$. Suppose that two random codewords, $\mathcal{C}_{1}$ and $\mathcal{C}_{2}$, are generated, and the coded bits are grouped in two sets, each set consists of 336 binary vectors, and each vector is of length 3. Suppose that out of the 336 binary vectors corresponding to $\mathcal{C}_{1}$ and $\mathcal{C}_{2}$, 4 binary vectors contain bits that correspond to the VNs of $T_{2}$. Let these binary vectors be $\Pi_{1}=\{01 \underline{0}, 0 \underline{1} 1,1 \underline{11}, 10 \underline{0}\}$ and $\Pi_{2}=\{01 \underline{0}, 0 \underline{0} 0,1 \underline{0} 0,11 \underline{0}\}$ for $\mathcal{C}_{1}$ and $\mathcal{C}_{2}$, respectively. From Fig. 4.1, the set of symbols corresponding to $\Pi_{1}$ and $\Pi_{2}$ are $\mathcal{S}_{1}=\left\{s_{2}, s_{3}, s_{7}, s_{4}\right\}$ and $\mathcal{S}_{2}=\left\{s_{2}, s_{0}, s_{4}, s_{6}\right\}$, respectively. From these sets, we notice that both have $s_{2}$ in the first VN. Hence, this VN has the same protection level in $\mathcal{C}_{1}$ and $\mathcal{C}_{2}$. For the fourth $V N, s_{4}$ is transmitted for $\mathcal{C}_{1}$ and $s_{6}$ is transmitted for $\mathcal{C}_{2}$. We note for these symbols, the VN corresponds to $B L 3$, i.e., the LSB. Hence, similar to the first VN, the fourth VN has the same protection level in $\mathcal{C}_{1}$ and $\mathcal{C}_{2}$. We now consider the second $V N$, which corresponds to BL 2. For this $V N$, the symbol $s_{3}$ is transmitted for $\mathcal{C}_{1}$ and the symbol $s_{0}$ is transmitted for $\mathcal{C}_{2}$. From Fig. 4.1, it can be seen that at BL 2, $s_{3}$ is more protected than $s_{0}$. A similar observation applies to the third $V N$, corresponding to $s_{7}$ for $\mathcal{C}_{1}$ and $s_{4}$ for $\mathcal{C}_{2}$. Hence, it can be seen that, despite the fact that both $\Pi_{1}$ and $\Pi_{2}$ correspond to $\pi_{T_{2}}$, they are protected differently by the constellation, effectively causing $\mathcal{C}_{2}$ to be more likely to be trapped in $T_{2}$ than $\mathcal{C}_{1}$.

The previous example suggests that the estimation of the EF for LDPC coded systems with PAM signalling depends on the particular codewords being transmitted. Hence, assuming that the all-zero codeword is transmitted throughout is not valid in this case. This further implies that the MS value in the MS-IS scheme must be adjusted to account for the various levels of error protection offered by each symbol in the constellation.

We conclude this section with a summary of the considerations that must be taken into account in order to evolve from estimating the EF in systems based on BPSK signalling to systems based on PAM signalling. This summary will serve as our guideline in developing the algorithm proposed in the next section.

1. The contributions of two isomorphic TSs to the EF of the code can be assumed equal only if they correspond to the same ordered BL sets; 
2. Using the fact that, conditioned on the transmitted symbol, the received signals are statistically independent, whereas the corresponding LLRs are not, renders the received signals more convenient in developing the prospective MSIS scheme; and

3. The MS values in the MS-IS scheme must be adjusted based on the constellation size, and the transmitted codeword.

\subsection{Proposed MS-IS scheme}

In this section, we use the guidelines developed in the previous section to propose a novel MS-IS scheme for estimating the EF of PAM-based LDPC-CMSs. Towards that end, we reconsider the summation in (4.14). To begin with, using Item 1 in Section 4.2.1, we modify the way that ETSs of each class are partitioned. Rather than considering all the isomorphic structures in one set as in the case of BPSK signalling, in the case of PAM signalling, the ETSs are identified only if they are isomorphic and have identical ordered BL sets. Such ETSs form what we refer to as an "equivalence class". Doing so yields the following expression for estimating the rate of decoding failure:

$$
\operatorname{Pr}(\mathcal{D} \mathcal{F}) \approx \sum_{(a, b) \in \mathcal{B}} \sum_{j=1}^{J_{a b}} \sum_{t=1}^{N_{a b, j}}\left|\mathcal{T}_{(a, b)}^{j, t}\right| \operatorname{Pr}\left(\mathcal{D F}, \mathrm{\top}_{(a, b)}^{j, t}\right)
$$

where $\mathcal{T}_{(a, b)}^{j, t} \subseteq \mathcal{T}_{(a, b)}^{j}$ is the $t$-th subset of $\mathcal{T}_{(a, b)}^{j}=\bigcup_{t} \mathcal{T}_{(a, b)}^{j, t}$, and the elements of $\mathcal{T}_{(a, b)}^{j, t}$ have identical ordered BL sets. The TS $\mathrm{T}_{(a, b)}^{j, t}$ is an arbitrary element of the set $\mathcal{T}_{(a, b)}^{j, t}$, and $N_{a b, j}$ is the total number of subsets $\left\{\mathcal{T}_{(a, b)}^{j, t}\right\}$ corresponding to distinct BL sets.

We note that to calculate the summation in (4.16), two challenges must be overcome. First, the probabilities $\operatorname{Pr}\left(\mathcal{D F}, \mathrm{T}_{(a, b)}^{j, t}\right)$ must be estimated for each $j \in\left\{1, \ldots, J_{a b}\right\}$ and $t \in\left\{1, \ldots, N_{a b, j}\right\}$. Second, the cardinalities $J_{a b}$ and $N_{a b, j}$ can be large, which implies that the evaluation of all the probabilities, $\operatorname{Pr}\left(\mathcal{D} \mathcal{F}, \mathrm{T}_{(a, b)}^{j, t}\right)$, can be computationally prohibitive. In the following, we will describe our proposed algorithms to alleviate these challenges. 


\subsubsection{Formulation of MS-IS for PAM Systems}

For ease of exposition, in the forthcoming discussion, we will drop the subscript $(a, b)$ and the superscript $(j, t)$, i.e., we focus on estimating $\operatorname{Pr}(\mathcal{D F}, T)$ for a given ETS, T. The development of the desired MS-IS scheme requires two main steps. First, we will formulate the MS-IS scheme for PAM systems, which includes deriving the biased and unbiased PDFs of the received signals rather than the LLRs, as per the discussion in Section 4.2.1, and obtaining a new weight function, the PAM counterpart of (4.13), which will be subsequently used to obtain an estimate of the probability of decoder failure. Second, we will provide a methodology for calculating the appropriate MS values in order to bias the received signal corresponding to every possible transmitted codeword. This is in contrast with the case of BPSK signalling in which it suffices to consider the all-zero codeword.

To use (4.6) to estimate $\operatorname{Pr}(\mathcal{D F}, T)$ for a PAM-based system, initially, the joint PDFs of the biased received signals, denoted by $\boldsymbol{Z}$, and the unbiased received signals, denoted by $\boldsymbol{R}$, for a given transmitted codeword must be derived. Note that both $\boldsymbol{R}$ and $\boldsymbol{Z}$ are deterministic functions of the transmitted codeword and noise. The joint PDF of $\boldsymbol{R}$ corresponding to a given transmitted codeword, $\mathcal{C}$, is denoted by $f_{\boldsymbol{R} \mid \mathcal{C}}(\boldsymbol{r})=\prod_{k=1}^{N_{s}} f_{R_{k}}\left(r_{k}\right)$, where $R_{k} \sim \mathcal{N}\left(s_{k}, \frac{N_{0}}{2}\right)$, and $N_{s}$ is the number of constellation symbols transmitted per codeword, cf. Section 2.5. In an analogous fashion the joint PDF of $\boldsymbol{Z}$ corresponding to the transmitted codeword, $\mathcal{C}$, can be expressed as

$$
f_{\boldsymbol{Z} \mid \mathcal{C}}(\boldsymbol{z})=\left(\prod_{k \in \zeta_{\boldsymbol{T}}} f_{R_{k}}\left(z_{k}-\mu_{\mathrm{\top}}^{v(k)}\right)\right)\left(\prod_{k \in\left\{1, \ldots, N_{s}\right\} \backslash \zeta_{\mathrm{T}}} f_{R_{k}}\left(z_{k}\right)\right),
$$

where, as per the definition in Section 4.1.2, $\zeta_{\mathrm{T}}=\left\{\zeta_{\mathrm{T}}^{v}\right\}_{v=1}^{a}$ is the ordered set of indices of the symbols mapped to VNs in T. We recall that, for BPSK, the sets T and $\zeta_{T}$ are identical. However, in higher-order modulation, the set $\zeta_{T}$ can be obtained from $T$ by taking into consideration the number of bits per symbol. For instance, consider $\mathrm{T}=\{396,467,548,570\}$ (as in Example 2) when a 16-PAM constellation is used. In this case, the number of bits per symbol is 4 and the $v$-th element of $\zeta_{\mathrm{T}}, \zeta_{\mathrm{T}}^{v}$ is given by $\left\lceil\frac{\mathrm{T}(v)}{4}\right\rceil$, where $\mathrm{T}(v)$ is the $v$-th element of $\mathrm{T}$. Hence, $\zeta_{\boldsymbol{T}}=\{99,117,137,143\}$. In (4.17), the $v$-th element of $\left\{\mu_{\mathrm{T}}^{v(k)}\right\}_{v=1}^{a}$ is the MS corresponding to the transmitted symbol 
indexed by $\zeta_{\mathrm{T}}^{v}$, that is $k$ is the $v(k)$-th element of $\zeta_{\mathrm{T}}$. For instance, in the foregoing example, $k=117$ corresponds to the second element of $\zeta_{\mathrm{T}}$ and hence $v(117)=2$.

Using the biased and unbiased PDFs, it can be readily seen that the weight function corresponding to $\mathcal{C}$ can be expressed as:

$$
w_{\mathcal{C}}(\boldsymbol{z})=\frac{f_{\boldsymbol{R} \mid \mathcal{C}}(\boldsymbol{z})}{f_{\boldsymbol{Z} \mid \mathcal{C}}(\boldsymbol{z})}=\prod_{k \in \zeta_{\mathrm{T}}} \frac{f_{R_{k}}\left(z_{k}\right)}{f_{R_{k}}\left(z_{k}-\mu_{\mathrm{T}}^{v(k)}\right)} .
$$

Using this expression for the weight function, the IS estimate for $\operatorname{Pr}(\mathcal{D} \mathcal{F}, T)$ can be obtained using a formula analogous to (4.6). In particular, by replacing the LLRs with biased received signals we have:

$$
\begin{aligned}
\operatorname{Pr}(\mathcal{D F}, \boldsymbol{\top}) & =\mathcal{E}_{\boldsymbol{Z}}\left\{w_{\mathcal{C}}(\boldsymbol{z}(\mathcal{C}, W)) \mathbb{I}_{\boldsymbol{R}_{E}}(\boldsymbol{z}(\mathcal{C}, W))\right\} \\
& \approx \frac{1}{N_{I S}} \sum_{i=1}^{N_{I S}} w_{\mathcal{C}}^{(i)}(\boldsymbol{z}(\mathcal{C}, W)) \mathbb{I}_{\boldsymbol{R}_{E}}^{(i)}(\boldsymbol{z}(\mathcal{C}, W)) .
\end{aligned}
$$

In (4.19), $\boldsymbol{R}_{E}$ is the union of all $N_{s}$-tuple received signals which cause the decoder to be trapped in $\mathrm{T}$. This region is equivalent to the error region, $\boldsymbol{L}_{E}$, parameterized by the LLRs in Section 4.1. The indicator function $\mathbb{I}_{\boldsymbol{R}_{E}}^{(i)}(\boldsymbol{z}(\mathcal{C}, W))=1$ if $\boldsymbol{z}(\mathcal{C}, W) \in \boldsymbol{R}_{E}$ and zero otherwise. We note that $w_{\mathcal{C}}^{(i)}(\boldsymbol{z})$ is the weight function corresponding to the $i$-th trial, in which the $i$-th randomly generated codeword, denoted by $\mathcal{C}$, is transmitted.

Analogous to (4.9), the variance of normalized error of estimation yielded by the IS scheme for all the TSs represented by $\mathrm{T}$, i.e., $\mathcal{T}_{(a, b)}^{j, t}$ can be obtained using the weight function in (4.18) yielding

$$
\gamma_{I S}(\mathbf{T})=\frac{1}{N_{I S}}\left(\frac{\mathcal{E}_{\boldsymbol{Z}}\left\{\left(w_{\mathcal{C}}^{(i)}(\boldsymbol{z}) \mathbb{I}_{\boldsymbol{R}_{E}}^{(i)}(\boldsymbol{z})\right)^{2}\right\}}{\operatorname{Pr}(\mathcal{D} \mathcal{F}, \mathbf{T})^{2}}-1\right)
$$

where in writing (4.21), we have replaced $P_{E}$ in (4.9) with $\operatorname{Pr}(\mathcal{D} \mathcal{F}, \mathbf{T})$.

\subsubsection{Methodology for Calculating the MS Values}

In the previous section we provided a formulation for the MS-IS scheme in the case of PAM signalling. This formulation depends on the MSs, $\left\{\mu_{\mathrm{T}}^{v(k)}\right\}$, that must be 
used to compute the biased PDFs corresponding to each TS, T. In this section, we will propose a methodology for calculating these MSs. We begin by making two definitions.

Definition 1 (Neighbor Zone) The neighbor zone of a given point s in a constellation $\Gamma, \mathcal{V}_{s}$, is given by

$$
\mathcal{V}_{s}=\left\{x \mid\|x-s\|_{2} \leq\|x-\hat{s}\|_{2}, \forall \hat{s} \neq s, s, \hat{s} \in \Gamma\right\}
$$

For implementing the MS-IS scheme for a given TS, $T$, of size $a$, the symbols to which the bits, i.e., the VNs, of this TS are mapped must be biased in a particular way, see Example 4. These bits will be referred to as "target bits", and to identify them, let $\pi_{T}$ be the BL set corresponding to a TS, T (cf. Example 2) and let $\mathcal{S}_{T}$ be the set of symbols corresponding to the VNs of $T$. The $q$-th element in $\pi_{T}$ and $\mathcal{S}_{T}$ are denoted by $\pi_{T}^{q}$ and $s_{T}^{q}$, respectively, and the cardinality of both sets is $a$, i.e., $\left|\pi_{T}\right|=\left|\mathcal{S}_{T}\right|=a$. Then, we have the following definition.

Definition 2 (Target Bit) The target bit within the $m$-bits that correspond to the symbol $s_{T}^{q}$ of the TS T is the $\pi_{T}^{q}$-th bit.

The following example illustrates how the target bits are determined.

Example 5 Consider the TS $T_{2}=\{396,467,548,570\}$ of the MacKay $(1008,504)$ LDPC code considered in Example 2 when 32-PAM signalling is used. The set of symbols corresponding to $T_{2}$ is denoted by $\mathcal{S}_{T_{2}}=\left\{s_{T_{2}}^{q}\right\}_{q=1}^{4}$, which depends on the transmitted codeword. As in Example 2, the BL set of $T_{2}$ is given by $\pi_{T_{2}}=\{1,2,3,5\}$, and hence from Definition 2 the target bits for the elements of $\mathcal{S}_{T_{2}}$ are 1-st bit of $s_{T_{2}}^{1}$, the 2-nd bit of $s_{T_{2}}^{2}$, the 3-rd bit of $s_{T_{2}}^{3}$, and the 5-th bit of $s_{T_{2}}^{4}$.

Using Definitions 1 and 2, we now describe our methodology in calculating the MS vector. We note that the purpose of shifting the mean of noise samples is to bias the LLRs of the target bits in $\mathcal{S}_{T}$. In particular, consider the symbol $s_{T}^{q} \in \mathcal{S}_{T}$, for which the $\pi_{T}^{q}$-th bit is the target bit. We seek an MS value which ensures that the received 
signal corresponding to $s_{T}^{q} \in \mathcal{S}_{T}$ falls in a region of the constellation where, with high probability, the two following conditions are met: 1) The sign of the LLR of $\pi_{T}^{q}$-th bit (target bit) is reversed; and 2) the signs of the LLRs of other $m-1$ bits remain unchanged. To satisfy these two constraints, we note that in an AWGN channel the probability of receiving a transmitted symbol in its neighbor zone is highest compared to any other location on the constellation. We use this observation to obtain the MS value which satisfies the aforementioned conditions when biasing the signals.

In particular, let the length- $m$ vector corresponding to the bits mapped to symbol $s_{T}^{q}$ be denoted by $\boldsymbol{b}_{s_{T}^{q}}$. To bias the transmitted symbol, $s_{T}^{q}$, so that the target bit is reversed whilst the remaining $(m-1)$ remain unchanged, we flip the $\pi_{T}^{q}$-th bit of $\boldsymbol{b}_{s_{T}^{q}}$, which yields a new vector $\overline{\boldsymbol{b}}_{s_{T}^{q}}$. This new vector corresponds to a unique constellation point, $\bar{s}_{T}^{q}$. Hence, the desired MS causes the mean of the received signal to be equal to $\bar{s}_{T}^{q}$ rather than $s_{T}^{q}$. This implies that, for every transmitted symbol in $\mathcal{S}_{T}, s_{T}^{q}$, the mean of the corresponding noise sample must be set to $\bar{s}_{T}^{q}-s_{T}^{q}$. That is, the MS of the noise sample corresponding to transmitted symbol $s_{T}^{q}$ is given by

$$
\mu_{s_{T}}^{q}=\bar{s}_{T}^{q}-s_{T}^{q}, \quad \forall s_{T}^{q} \in \mathcal{S}_{T}
$$

It is worth noting that the MS in (4.22) is not unique since any MS that causes the received signal to fall within the neighboring zone of $\bar{s}_{T}^{q}$ meets the aforementioned conditions. Hence, the MS value can be fine-tuned for more efficient implementation. In particular, the MS calculated in (4.22) can be reduced by $\hat{\nu}$, where $0<\hat{\nu} \leq d / 2$, and $d$ is the minimum distance of the constellation. The constraint $0<\hat{\nu} \leq d / 2$ aims at keeping the biased received signals within the neighbor zone of $\bar{s}_{T}^{q}$ with high probability. Reducing $\mu_{s_{T}}^{q}$ by $\hat{\nu}$ makes the value of the unbiased PDF at the received signal larger, which in turn increases the weight function value. To elaborate, note that to calculate the weight function, we need to replace the biased received signal in the biased and unbiased PDFs. Reducing $\mu_{s_{T}}^{q}$ by $\hat{\nu}$ moves the mean of the noise toward the unbiased symbol. The unbiased PDF is Gaussian with mean $s_{T}^{q}$; thus, the term $\hat{\nu}$ results in the samples of the biased distribution become closer to the mean of unbiased PDF, $s_{T}^{q}$, and consequently increases the unbiased PDF value at the biased received signal. It should be noted that the parameter $\hat{\nu}$ does not affect the biased 
PDF value. For a given LDPC code and a given constellation size, the optimal value of $\hat{\nu}$ can be obtained empirically by running the simulation for different $\hat{\nu}$ values.

For a given PAM constellation size, and for a given TS, the proposed methodology of MS calculation is summarized in the following algorithm.

Algorithm 1 Calculating the MS Values of the Noise Samples for the Symbols Corresponding to a Given TS.

Input: TS $(T)$, \# of bits per symbol $(m)$, set of symbols to which the VNs of the TS are mapped $\left(\mathcal{S}_{T}=\left\{s_{T}^{v}\right\}_{v=1}^{a}\right)$.

Output: Vector of MSs, $\boldsymbol{\mu}_{\mathcal{S}_{T}}=\left[\mu_{s_{T}^{1}}, \ldots, \ldots, \mu_{s_{T}^{a}}\right]$

1: for $v=1$ to $a$ do

2: Obtain $\boldsymbol{b}_{s_{T}^{v}}$, the vector of BLs corresponding to $s_{T}^{v}$, i.e., $\boldsymbol{b}_{s_{T}^{v}}=$ $\left[b_{v 1}, \ldots, b_{v \rho}, \ldots, b_{v m}\right]$.

3: $\quad$ Let $b_{v \rho}$ be the target bit for the symbol $s_{T}^{v}$, cf. Definition 2.

4: $\quad$ Let $\bar{b}_{v \rho}=b_{v \rho} \oplus 1$, where $\oplus$ shows the modulo 2 addition operator.

5: $\quad$ Replace $b_{v \rho}$ with $\bar{b}_{v \rho}$ in $\boldsymbol{b}_{s_{T}^{v}}$ to form $\overline{\boldsymbol{b}}_{s_{T}^{v}}=\left[b_{v 1}, \ldots, \bar{b}_{v \rho}, \ldots, b_{v m}\right]$.

6: $\quad$ Find the constellation symbol, $\bar{s}_{T}^{v}$, to which $\overline{\boldsymbol{b}}_{s_{T}^{v}}$ is mapped.

7: $\quad$ Set $\mu_{s_{T}^{v}}=\bar{s}_{T}^{v}-s_{T}^{v}$.

8: end for

\subsubsection{Identifying the PDTSs out of the $N_{a b, j}$ Partitions of $\mathcal{T}_{(a, b)}^{j}$}

In Section 4.3.1, we presented the proposed MS-IS scheme to estimate $\operatorname{Pr}\left(\mathcal{D} \mathcal{F}, \mathrm{T}_{(a, b)}^{j, t}\right)$, i.e., the error rate contribution of an arbitrary $\mathrm{TS}, \mathrm{T}_{(a, b)}^{j, t}$, in the PAM-based LDPCCMS. Our goal now is to use (4.16) to obtain an estimate of $\operatorname{Pr}(\mathcal{D} \mathcal{F})$. However, direct computation of the right hand side of (4.16) is costly and unfortunately grows with the size of the constellation and the length of the code. To alleviate this difficulty, we note that the total number of times that $\operatorname{Pr}\left(\mathcal{D} \mathcal{F}, \mathrm{T}_{(a, b)}^{j, t}\right)$ is estimated in (4.16) is determined by the following three parameters: 1) the number of TS classes considered in the summation, i.e., $|\mathcal{B}|, 2)$ the number of isomorphic subsets within a given $(a, b)$ class, i.e., $J_{a b}$, and 3) the number of subsets with identical BLs within each isomorphic set, i.e., $N_{a b, j}$.

The first two parameters, i.e., $|\mathcal{B}|$ and $J_{a b}$, depend solely on the code and are 
otherwise invariant to the size of the underlying constellation. In contrast, the third parameter, i.e., $N_{a b, j}$, depends on the size of the constellation through the number of bits that are mapped to each symbol. Hence, one way to reduce the computational burden of estimating $\operatorname{Pr}(\mathcal{D} \mathcal{F})$ is to find a technique whereby the subsets with identical BLs within each isomorphic set that make negligible contributions to the summation in (4.16) are eliminated from the computation. This approach reduces the upper limit of the innermost summation in (4.16) from $N_{a b, j}$ to a smaller integer, which subsequently results in a significant reduction in the overall computation.

To identify the BL sets with negligible contribution to the summation (4.16), we will establish a relationship between the probability of decoding failure, $\operatorname{Pr}(\mathcal{D} \mathcal{F}, T)$, and the BL set of the TS T. This relationship will enable us to identify the most influential BL sets. Towards that end, let $\pi_{\mathrm{T}}$ be the BL set corresponding to $\mathrm{T}$. Using (4.20), the probability of decoding failure can be written as:

$$
\begin{aligned}
\operatorname{Pr}(\mathcal{D} \mathcal{F}, \boldsymbol{\top}) & =\frac{1}{N_{I S}} \sum_{i=1}^{N_{I S}} w_{\mathcal{C}}^{(i)}(\boldsymbol{z}) \mathbb{I}_{\boldsymbol{R}_{E}}^{(i)}(\boldsymbol{z}) \\
& \approx \frac{1}{N_{I S}} \sum_{i=1}^{N_{I S}} w_{\mathcal{C}}^{(i)}(\boldsymbol{z}) .
\end{aligned}
$$

In (4.23), we assume that biasing the mean will almost surely cause the decoding failure, i.e., $\mathbb{I}_{\boldsymbol{R}_{E}}^{(i)}(\boldsymbol{z})=1$. For the weight function in (4.23) we note that:

$$
\begin{aligned}
w_{\mathcal{C}}(\boldsymbol{z}) & =\exp \left[\frac{1}{N_{0}} \sum_{k \in \zeta_{\mathrm{T}}}\left(z_{k}-\mu_{\mathrm{T}}^{v(k)}-s_{\mathrm{T}}^{v(k)}\right)^{2}-\left(z_{k}-s_{\mathrm{T}}^{v(k)}\right)^{2}\right] \\
& =\exp \left[\frac{1}{N_{0}} \sum_{k \in \zeta_{\mathrm{T}}} \mathrm{w}_{k}^{2}-\left(\mathrm{w}_{k}+\mu_{\mathrm{T}}^{v(k)}\right)^{2}\right] \\
& =\exp \left[\frac{1}{N_{0}} \sum_{k \in \zeta_{\mathrm{T}}} \mathrm{w}_{k}^{2}-\left(\mathrm{w}_{k}+\bar{s}_{\mathrm{T}}^{v(k)}-s_{\mathrm{T}}^{v(k)}\right)^{2}\right],
\end{aligned}
$$

where in writing (4.24) we used the fact that $z_{k}=s_{\mathrm{T}}^{v(k)}+\mathrm{w}_{k}+\mu_{\mathrm{T}}^{v(k)}$ and (4.25) is obtained by substituting for $\mu_{\mathrm{T}}^{v(k)}$ from (4.22). To obtain an estimate of $\sum_{i=1}^{N_{I S}} w_{\mathcal{C}}^{(i)}(\boldsymbol{z})$, we will invoke two approximations. In the first approximation, we consider the case 
of high SNRs, which will enable us to compare weights based on the distance between points in the constellation. In the second approximation, we neglect the impact of the weights that make negligible contribution to the computation of $\operatorname{Pr}(\mathcal{D} \mathcal{F}, T)$.

For the first approximation, we partition the $N_{I S}$ weights in the summation in (4.23) into two categories. The first category includes the weights in which the symbols $s_{\mathrm{T}}^{v(k)}$ and $\bar{s}_{\mathrm{T}}^{v(k)}$ have the least Euclidean distance.

We denote the number of such weights by $\hat{N}_{\mathrm{T}}$. The second category includes the remaining $N_{I S}-\hat{N}_{\mathrm{T}}$ weights. Using this categorization we have:

$$
\sum_{i=1}^{N_{I S}} w_{\mathcal{C}}^{(i)}(\boldsymbol{z})=\sum_{i=1}^{\hat{N}_{\mathrm{T}}} w_{\mathcal{C}}^{(i)}(\hat{\boldsymbol{z}})+\sum_{i=\hat{N}_{\mathrm{T}}+1}^{N_{I S}} w_{\mathcal{C}}^{(i)}(\boldsymbol{z}),
$$

where $\hat{\boldsymbol{z}} \triangleq\left\{\boldsymbol{z}\left|\bar{s}_{\mathrm{T}}^{v(k)}=\arg \min _{s}\right| s_{\mathrm{T}}^{v(k)}-\bar{s}_{\mathrm{T}}^{v(k)} \mid, \forall k \in \zeta_{\mathrm{T}}\right\}$. Since the least Euclidean distance between two constellation points is constant, at high SNRs, the noise $\mathrm{w}_{k}$ in $(4.25)$ becomes negligible and $w_{\mathcal{C}}^{(i)}(\hat{\boldsymbol{z}}) \approx \exp \left(\frac{-a d^{2}}{N_{0}}\right)$, where $d$ is the minimum Euclidean distance between points in the constellation. Using this observation, the summation in (4.26) can be approximated by

$$
\sum_{i=1}^{N_{I S}} w_{\mathcal{C}}^{(i)}(\boldsymbol{z}) \approx \hat{N}_{\mathrm{T}} \exp \left(\frac{-a d^{2}}{N_{0}}\right)+\sum_{i=\hat{N}_{\mathrm{T}}+1}^{N_{I S}} w_{\mathcal{C}}^{(i)}(\boldsymbol{z}) .
$$

For the second approximation, we note that because $w_{\mathcal{C}}^{(i)}(\boldsymbol{z})$ in the second summation correspond to distances between constellation symbols that are strictly greater than $d$, and since the exponent in the expression of $w_{\mathcal{C}}^{(i)}(\boldsymbol{z})$ in (4.25) depends quadratically on this distance, we have that, at high $\operatorname{SNRs}, w_{\mathcal{C}}^{(i)}(\boldsymbol{z}) \ll \exp \left(\frac{-a d^{2}}{N_{0}}\right)$. Hence, we conclude that the contribution of the second summation is negligible in computing the left hand side of (4.27). Using this approximation and by combining (4.27) and (4.23) we have:

$$
\operatorname{Pr}(\mathcal{D} \mathcal{F}, \mathrm{\top}) \approx \frac{\hat{N}_{\mathrm{T}}}{N_{I S}} \exp \left(\frac{-a d^{2}}{N_{0}}\right) .
$$

From (4.28), it is implied that the transmitted codewords corresponding to the symbol pairs $\left(s_{\mathrm{T}}^{v(k)}, \bar{s}_{\mathrm{T}}^{v(k)}\right)$ such that $\left|s_{\mathrm{T}}^{v(k)}-\bar{s}_{\mathrm{T}}^{v(k)}\right|=d$ make a significant contribution to the EF. To identify these codewords from the rest of the codewords, we let $\boldsymbol{b}^{(s)}$ be the 
$m$-dimensional binary vector corresponding to the symbol $s$, and let the $i$-th element of $\boldsymbol{b}^{(s)}$ be denoted by $\boldsymbol{b}^{(s)}(i)$ and $\boldsymbol{b}^{(s)}$ is mapped to constellation symbol $s$. Using this notation, we make the following definitions for a given TS, T, with a corresponding BL set, $\pi_{\mathrm{T}}$.

Definition 3 (Weak Symbol at the $\pi_{\mathrm{T}}^{k}$-th BL) The symbol s is defined as a weak symbol at the $\pi_{\mathrm{T}}^{k}$-th $B L$ if there exists an adjacent symbol to $s$, denoted by $\bar{s}$, such that $\boldsymbol{b}^{(s)}\left(\pi_{\mathrm{T}}^{k}\right)=\boldsymbol{b}^{(\bar{s})}\left(\pi_{\mathrm{T}}^{k}\right) \oplus 1$ and $\boldsymbol{b}^{(s)}(i)=\boldsymbol{b}^{(\bar{s})}(i), \forall i \neq \pi_{\mathrm{T}}^{k}$.

Using this definition, we can characterize the codewords for which all the symbols corresponding to $\mathrm{T}$ are weak in the positions given in the corresponding $\mathrm{BL}$ set. We refer to such codewords as [T-weak], which are formally defined as follows.

Definition 4 (T-Weak Codewords) A T-weak codeword is defined as the codeword for which all the elements in $\mathcal{S}_{\mathrm{T}}$ are weak at the BLs corresponding to $\mathrm{T}$, i.e., they are weak at the BLs determined by the elements of $\pi_{\mathrm{T}}$.

From this definition, it can be seen that the $\hat{N}_{\mathrm{T}}$ in (4.28) is precisely the number of T-weak codewords among $N_{I S}$ codewords considered. Hence, the fraction $\frac{\hat{N}_{\mathrm{T}}}{N_{I S}}$ can be interpreted as the probability of having $\hat{N}_{\mathrm{T}}$ T-weak codewords in the $N_{I S}$ trials. Using this observation, (4.28) can be expressed as follows:

$$
\operatorname{Pr}(\mathcal{D F}, \mathrm{T}) \approx \operatorname{Pr}(\mathrm{T} \text {-weak codeword }) \exp \left(\frac{-a d^{2}}{N_{0}}\right)
$$

From (4.29), it is observed that the probability of decoding failure is proportional to both $\operatorname{Pr}\left(\mathrm{T}\right.$-weak codeword) and $\exp \left(\frac{-a d^{2}}{N_{0}}\right)$. However, the second term depends solely on the minimum distance of the constellation, but not on the BL sets. In contrast, we will show that the first term, i.e., $\operatorname{Pr}(\mathrm{T}$-weak codeword), is intimately related to the BL sets. In particular, in Appendix B.1 we prove that

$$
\operatorname{Pr}(\mathrm{T} \text {-weak codeword })=\prod_{i=1}^{a} 2^{\left(\pi_{\mathrm{T}}^{i}-m\right)}
$$

whence we have

$$
\operatorname{Pr}(\mathcal{D} \mathcal{F}, \boldsymbol{\top}) \approx \exp \left(\frac{-a d^{2}}{N_{0}}\right) \prod_{i=1}^{a} 2^{\left(\pi_{\mathrm{T}}^{i}-m\right)}
$$


which establishes the connection between $\operatorname{Pr}(\mathcal{D} \mathcal{F}, T)$ and the structure of the BL set corresponding to T. Using (4.31) enables us to obtain the relative contribution of different BL sets and to decide whether a BL set is included in the EF estimation or it is ignored. To clarify this comparison further, we provide the following example.

Example 6 We estimate the error rate corresponding to a TS class (6,2) when 32-PAM signalling is used. The TSs are partitioned in different BL sets. According to (4.31) for the $B L$ set, $\pi_{\mathrm{T}}$, represented by $\mathrm{T}$ we have $2^{-24} \exp \left(\frac{-a d^{2}}{N_{0}}\right) \leq$ $\operatorname{Pr}(\mathcal{D F}, \mathrm{T}) \leq \exp \left(\frac{-a d^{2}}{N_{0}}\right)$. More specifically, consider two BL sets $\pi_{\mathrm{T}_{1}}$ and $\pi_{\mathrm{T}_{2}}$ with $\operatorname{Pr}\left(\mathrm{T}_{1}\right.$-weak codeword $)=2^{-18}$ and $\operatorname{Pr}\left(\mathrm{T}_{2}\right.$-weak codeword $)=2^{-8}$. For the TSs belonging to $\pi_{\mathrm{T}_{1}}$ and $\pi_{\mathrm{T}_{2}}$, using (4.31) yields $\operatorname{Pr}\left(\mathcal{D} \mathcal{F}, \mathrm{T}_{2}\right)=2^{10} \times \operatorname{Pr}\left(\mathcal{D} \mathcal{F}, \mathrm{T}_{1}\right)$. In this case, the BL set $\pi_{\mathrm{T}_{1}}$ and all the corresponding TSs are removed from the innermost summation in (4.16) since their contribution is negligible.

We note that derivation of (4.31) invokes several approximations, which renders it rather crude for obtaining an estimate of the error floor. However, this equation provides valuable guidance in choosing the TSs that are likely to make significant contribution to the EF.

\subsubsection{Summary of the PDTS Extraction Technique}

In this section, we use the analysis in Section 4.3.3 to present our PDTS extraction technique. In particular, to extract the most influential BL sets, we consider the class $(a, b)$ TS and the $j$-th isomorphic subset in (4.16). We then use (4.30) to calculate $\operatorname{Pr}(\mathrm{T}$-weak codeword) for all the BL sets involved in the innermost summation in (4.16), i.e., $N_{a b, j}$ BL sets. To compare all the BL sets collectively, these probabilities are normalized with respect to their maximum value. By comparing the normalized probability with a prescribed threshold, which we denote by $\beta_{\mathcal{T}}^{\text {th }}$, we choose the BL sets that are considered in the calculation. In particular, any BL set for which the normalized probability of TS to be T-weak falls below this threshold is discarded. A summary of this technique is presented in Algorithm 2 below. 


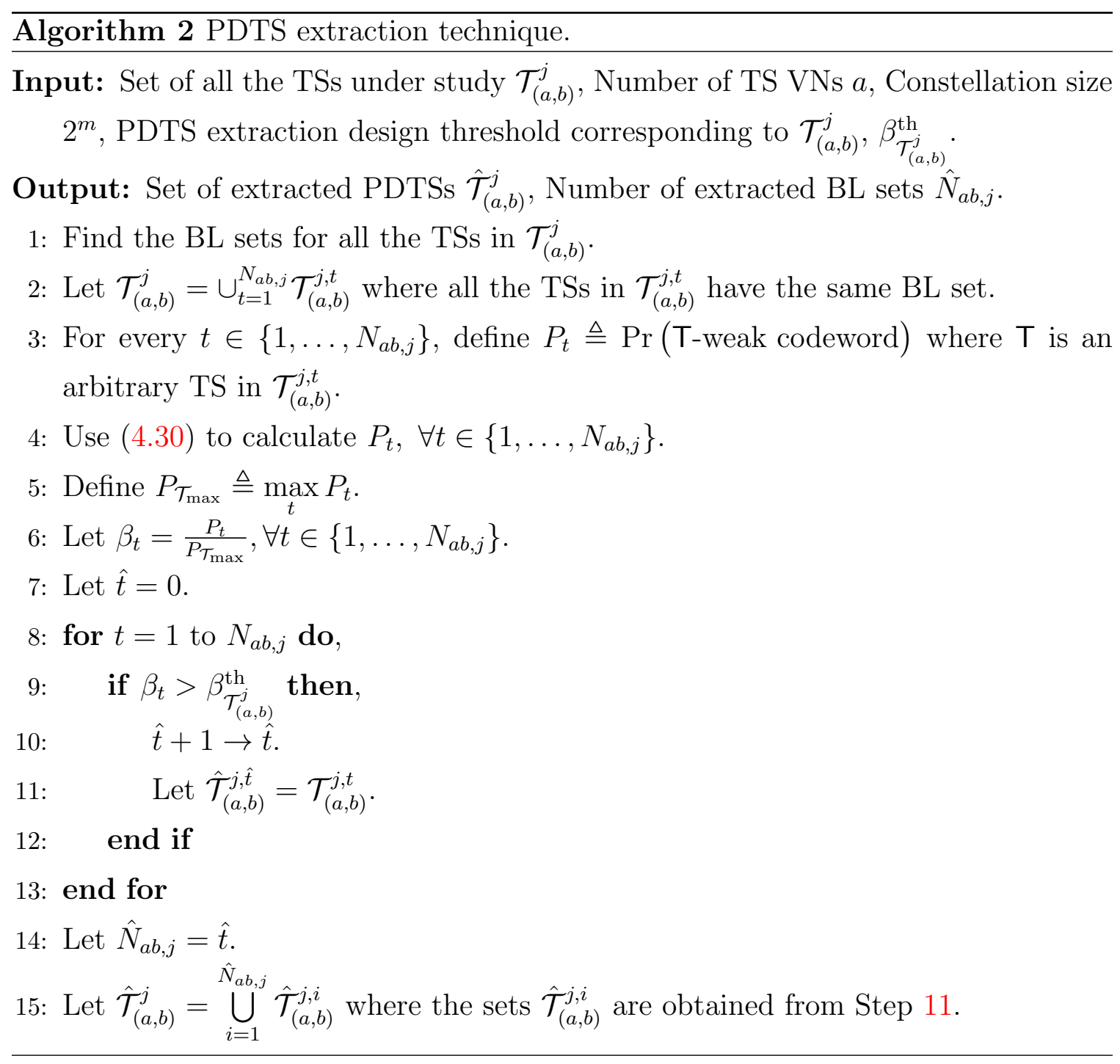

We choose the threshold, $\beta_{\mathcal{T}}^{\text {th }}$, empirically, and by making the following two observations: 1) For a TS class with $P_{\mathcal{T}_{\max }}$ (cf. Step 5 in algorithm 2) close to 1 , the probability of a random codeword being weak against the TSs of that class is high. Hence, in addition to the TSs corresponding to $P_{\mathcal{T}_{\max }}$, other TSs with $\operatorname{Pr}$ (T-weak codeword) less than $P_{\mathcal{T}_{\max }}$ have the chance of becoming dominant, and we need to loosen the selection criterion such that more BL sets are chosen. This is equivalent of selecting a smaller $\left.\beta_{\mathcal{T}}^{\text {th }}, 2\right)$ For a TS class with small $P_{\mathcal{T}_{\max }}$ (e.g., $P_{\mathcal{T}_{\max }}<10^{-3}$ ), all the TSs have a small chance of becoming dominant. Hence, even the TSs corresponding to $P_{\mathcal{T}_{\max }}$ are, by definition, unlikely to become dominant. Therefore, for BL sets with small $P_{\mathcal{T}_{\text {max }}}$, we choose large $\beta_{\mathcal{T}}^{\text {th }}$ values, which result in a smaller number of extracted TSs. 


\subsection{Summary of Proposed EF Estimation Scheme}

In this section, we summarize the steps required for implementing the proposed EF estimation scheme. In this scheme, the LDPC code, the SNR, the constellation size, and the reliability threshold of the estimates are considered as inputs. As Step 0, we perform the following tasks to find all the required TSs, for which the error rate contribution needs to be estimated by the proposed MS-IS scheme.

1. Use MC simulation to identify the size of involved TSs at the early EF region.

2. Set the parameter $a_{\max }$ and $b_{\max }$ to the largest TS class obtained from the previous step.

3. Form the set $\mathcal{B}$ that includes all the TS classes for which the error rate must be estimated, cf. (4.14).

4. Use the algorithms in [40] and [42] to obtain all the ETSs of each class in $\mathcal{B}$.

For each TS class, $(a, b)$, in $\mathcal{B}$ the following steps are carried out to estimate its error rate contribution. These contributions then are accumulated to estimate the EF.

1. Form $\mathcal{T}_{(a, b)}^{j}$ sets: partition the TSs into subsets with the isomorphic elements. Denote the $j$-th subset by $\mathcal{T}_{(a, b)}^{j}$, cf. (4.15). The number of such subsets is $J_{a b}$.

2. Form $\mathcal{T}_{(a, b)}^{j, t}$ sets: partition the TSs in $\mathcal{T}_{(a, b)}^{j}$ into subsets, in which the TSs have identical BL sets. Denote the $t$-th subset by $\mathcal{T}_{(a, b)}^{j, t}$, cf. (4.16). The number of such subsets is $N_{a b, j}$.

3. Apply the PDTS extraction technique described in Section 4.3 .3 on the $N_{a b, j}$ subsets in the previous step to extract $\hat{N}_{a b, j}$ BL sets.

4. Choose an arbitrary representative TS from $\mathcal{T}_{(a, b)}^{j, t}$. Denote this TS by T.

5. Generate a random codeword, $\mathcal{C}$.

6. Map the coded bits to constellation symbols and find the subset of symbols corresponding to $\mathrm{T}$, i.e., $\mathcal{S}_{\mathrm{T}}$.

7. Calculate the vector of MS values, $\boldsymbol{\mu}_{\mathcal{S}_{\top}}$, cf. Section 4.3.2. 
8. Shift the noise of symbols in $\mathcal{S}_{\mathrm{T}}$ by MS values in $\boldsymbol{\mu}_{\mathcal{S}_{\mathrm{T}}}$.

9. For the transmitted symbols corresponding to $\mathcal{C}$, including the biased ones in $\mathcal{S}_{\mathrm{T}}$, generate the received signals.

10. Decode the received signals and check whether the decoder is trapped in $\mathrm{T}$.

11. Update the estimate of the error rate, $\operatorname{Pr}(\mathcal{D F}, T)$, using (4.20) and the parameter $\gamma_{I S}(\mathrm{~T})$, using $(4.21)$.

12. If $\gamma_{I S}(\mathbf{T})$ is more than the prescribed threshold go to step 5 .

13. If $t<\hat{N}_{a b, j}$, increase $t$ by 1 and go to step 4 .

14. If $j<J_{a b}$, increase $j$ by 1 and go to step 3 .

15. Use (4.16) to accumulate all the estimated error rates, $\operatorname{Pr}(\mathcal{D} \mathcal{F}, \boldsymbol{T})$, corresponding to all the $(j, t)$ values, to obtain an estimate of the error rate corresponding to class $(a, b)$ TSs.

\subsection{Simulation Results and Analysis}

In this section, we present the simulation results of proposed EF estimation scheme. In particular, the FER curves corresponding to the conventional MC simulation and the proposed MS-IS scheme are drawn. We consider Four widely used LDPC codes, i.e., the Tanner $(155,64)$ [81], the MacKay $(1008,504)$ [62], and two codes from the family of WiMAX LDPC code, namely WiMAX1 $(2016,1344)$ and WiMAX2 $(1152,960)$ LDPC codes [82]. The first two are regular codes while the third and the fourth codes are irregular quasi-cyclic (QC) codes with lifting degrees 84 and 48, respectively. We consider $M$-PAM signalling, where $M=2^{m}, m \in\{3, \ldots, 7\}$. The decoder is chosen to be the conventional min-sum one [83] with the following parameters: the number of quantization bits is set to 6, the maximum number of iterations is set to 100 , and the clipping (saturation) threshold of quantization for each case is stated on the corresponding figure's legend using the notation 'sat'. The parameter $\gamma_{I S}(\mathrm{~T})$ for both MC and MS-IS schemes is set to $5 \%$. 
To apply the PDTS extraction technique, the threshold $\beta_{\mathcal{T}}^{\text {th }}$ is set to the values given in Table 4.1 below. These values are found empirically using the following observation: in our simulations we observed that large $P_{\mathcal{T}_{\max }}$ values (cf. Algorithm 2) require more BL sets and thus smaller thresholds in the estimation. The impact of $\beta_{\mathcal{T}}^{\text {th }}$ on the performance of proposed MS-IS scheme will be investigated in Section. 4.5.1.

\begin{tabular}{|c|c|c|c|}
\hline$P_{\mathcal{T}_{\max }}$ & $P_{\mathcal{T}_{\max }} \geq 0.1$ & $0.1<P_{\mathcal{T}_{\max }} \leq 0.05$ & $0.05<P_{\mathcal{T}_{\max }} \leq 0.001$ \\
\hline$\beta_{\mathcal{T}}^{\text {th }}$ & 0.025 & 0.0375 & 0.05 \\
\hline$P_{\mathcal{T}_{\max }}$ & $0.001<P_{\mathcal{T}_{\max }} \leq 0.0005$ & $0.0005<P_{\mathcal{T}_{\max }} \leq 0.0001$ & $P_{\mathcal{T}_{\max }}<0.0001$ \\
\hline$\beta_{\mathcal{T}}^{\text {th }}$ & 0.125 & 0.1875 & 0.225 \\
\hline
\end{tabular}

Table 4.1: The empirical values of the threshold $\beta_{\mathcal{T}}^{\text {th }}$ in Algorithm 2

We begin the simulation by estimating the EF of the aforementioned codes using MC and proposed MS-IS scheme. We relegate the analysis of the results to Section. 4.5.1. For the Tanner $(155,64)$ LDPC code, we have 2 dominant TS classes, namely $(8,2)$ and $(10,2)[40]$. The MC simulation verifies that both classes are present when the non-binary constellations are used. Thus, we set $a_{\max }=10$ and $b_{\max }=2$. Figure 4.3 shows the MC and the MS-IS FER estimation results for this code when the PAM signalling is used. It can be seen that the MS-IS estimation and MC simulation curves give similar results. This shows that the proposed MS-IS estimation scheme can effectively estimate the FER in the EF region.

To further verify the effectiveness of the proposed MS-IS scheme, the FER curves for the MacKay $(1008,504)$ LDPC code using the MC simulation and the proposed MS-IS estimation schemes is displayed in Fig. 4.4. The MC simulation at the early EF region demonstrates that the class $(8,2)$ is the largest size TS class with nonnegligible FER. Hence, we set $a_{\max }=8$ and $b_{\max }=2$ to estimate the FER we should consider 3 TS classes, namely $(4,2),(6,2)$, and $(8,2)$. At the SNRs for which the results for both MC and MS-IS are available, it can be seen that both methods yield similar FER estimate.

For the WiMAX LDPC codes the FER curves are shown in Figs. 4.5 and 4.6. 


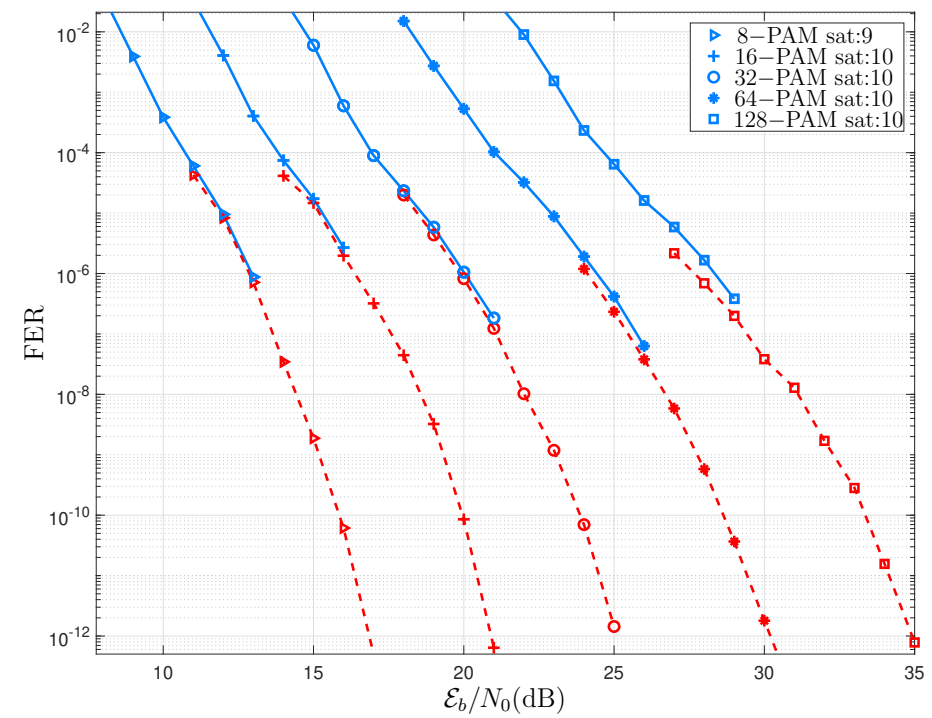

Figure 4.3: The MC (solid-line) and the MS-IS (dashed-line) simulation results for the Tanner $(155,64)$ LDPC code with $M-$ PAM signalling.

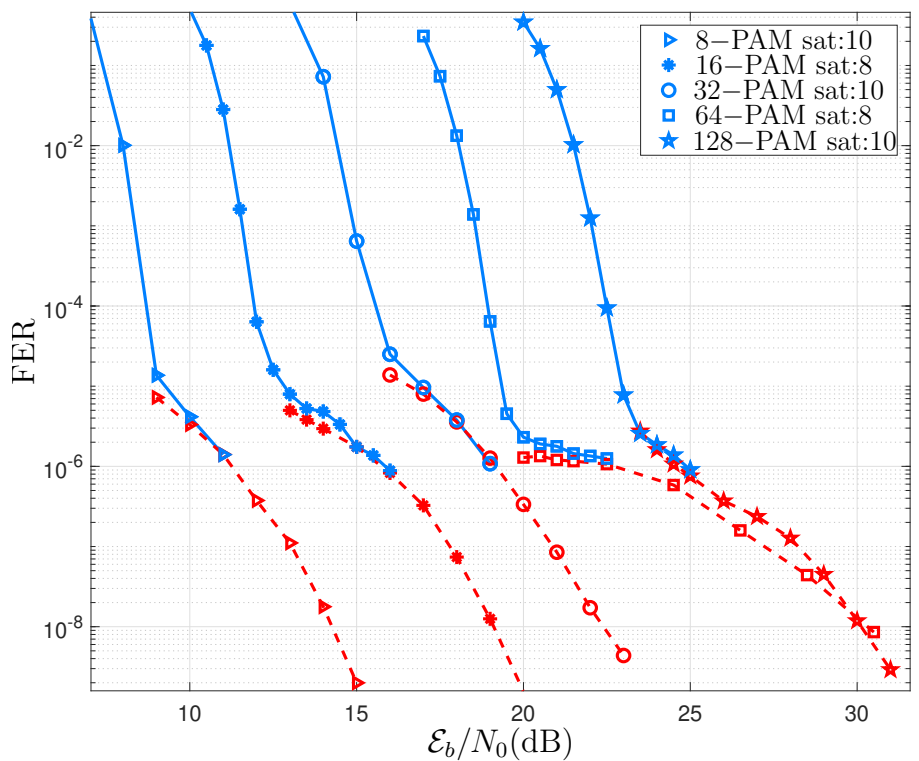

Figure 4.4: The MC (solid-line) and the MS-IS (dashed-line) simulation results for the MacKay $(1008,504)$ LDPC code with $M$-PAM signalling.

To select the TS classes for estimating the EF we use MC simulation results. The choices of $a_{\max }$ for each constellation size varies from one another. These TS sizes are displayed in Tables B.3 and B.4. In the EF estimation of the irregular codes, the constraint of the isomorphism was removed from the calculations. In other words, we categorize the TSs in each class according to the BL sets only. This is due to the 


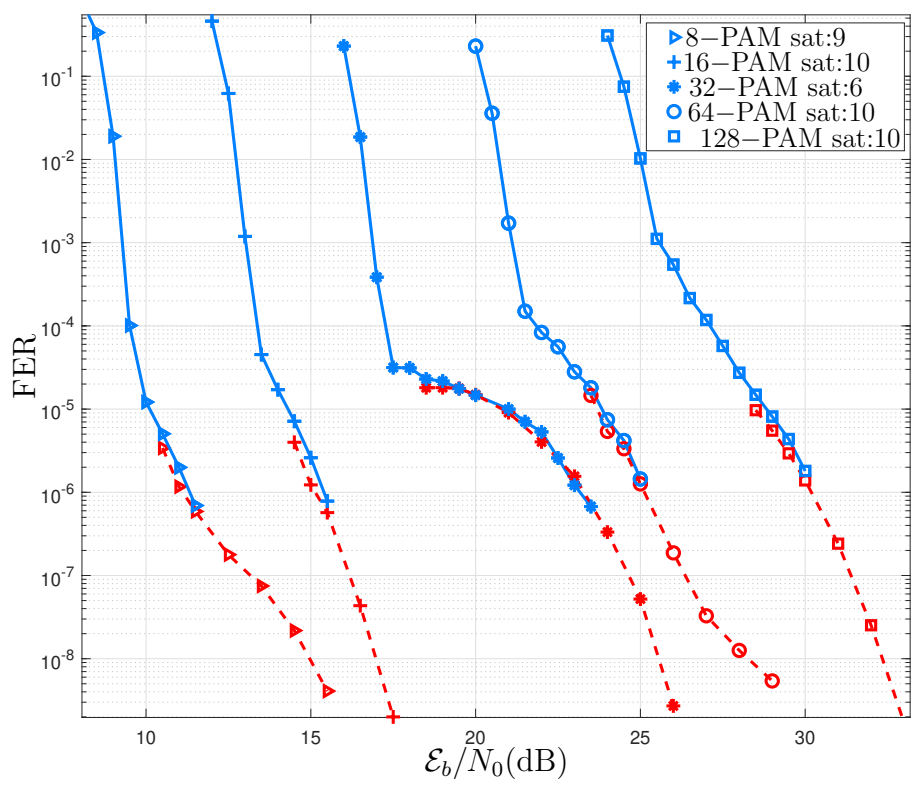

Figure 4.5: The MC (solid-line) and the MS-IS (dashed-line) simulation results for the WiMAX1 $(2016,1344)$ LDPC code with $M$-PAM signalling.

larger number of isomorphic structures in the Tanner graph of the irregular codes compared to the regular ones. These examples illustrate the effectiveness of proposed technique for estimating the EF in the case of using irregular LDPC codes with nonbinary signalling. As it is shown in Figs. 4.5 and 4.6, both schemes result in similar FER curves.

We note that the QC structure of these codes renders them amenable to further reduction in the computational complexity of estimating the EF. This is because for a QC-LDPC code, the TSs in one class can be partitioned into subsets such that within each subset, dividing the indices of VNs of TSs by $m$ results in an identical set of remainders. Compared to the random codes, this property will decrease the number of unique BL sets, $N_{a b, j}$ in (4.16), i.e., the number of partitioned TS candidates in each class.

\subsubsection{Discussion and Interpretation of Numerical Results}

Figs. 4.3-4.6 attest to the effectiveness of the proposed MS-IS scheme in estimating the EF of regular and irregular LDPC coded systems with non-binary PAM signalling. 


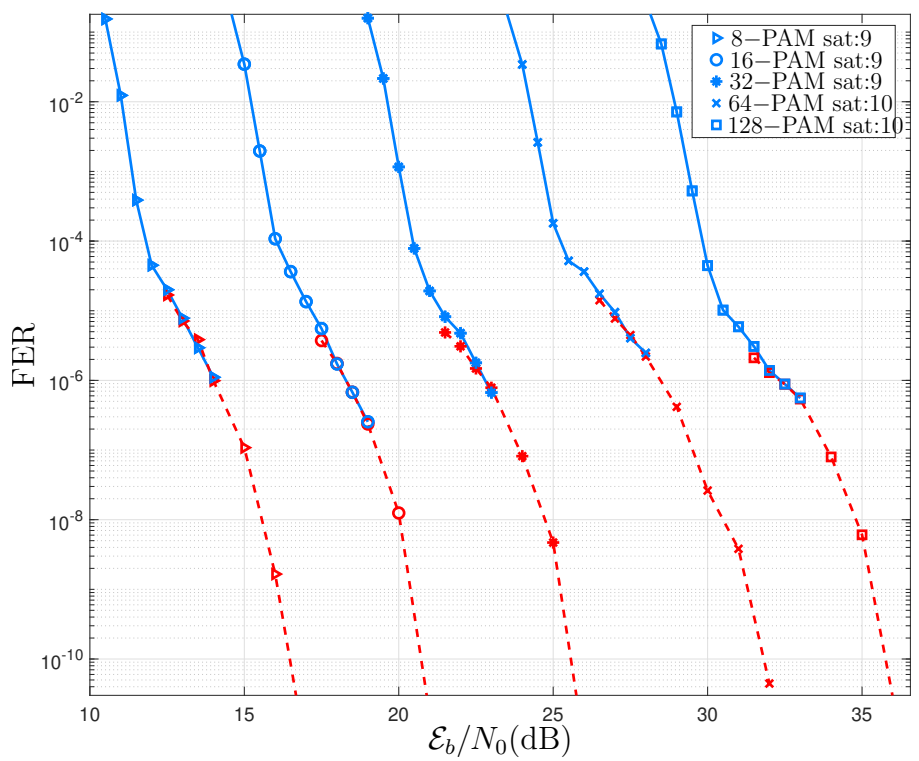

Figure 4.6: The MC (solid-line) and the MS-IS (dashed-line) simulation results for the WiMAX2 $(1152,960)$ LDPC code with $M$-PAM signalling.

We now investigate the performance of the proposed MS-IS scheme from different aspects in more detail. To perform this investigation in Table 4.2 we provide detailed information on the simulation results presented in Fig. 4.3. Although these results correspond to the Tanner $(155,64)$ code, similar trends are observed in the case of other codes including those simulated in Figs. 4.4 and 4.6. The tables corresponding to the latter figures are shown in Appendix B.2. Five aspects will be considered: 1) the performance of PDTS extraction technique presented in Section. 4.3.3, 2) the effect of the threshold $\beta_{\mathcal{T}}^{\text {th }}$ in Algorithm 2 on the estimation performance, 3 ) the comparison of time efficiency between the MC scheme and the proposed MS-IS scheme, 4) the number of required trials in the MS-IS scheme, and 5) the number of PDTSs corresponding to a given TS class.

1. Performance of PDTS extraction technique: To illustrate the effect of this technique, in Table 4.2 we show the numerical value of $\sum_{j=1}^{J_{a b}} \sum_{t=}^{\hat{N}_{a b, j}}\left|\hat{\mathcal{T}}_{(a, b)}^{j, t}\right|$, where $\hat{N}_{a b, j}$, and $\hat{\mathcal{T}}_{(a, b)}^{j, t}$ are calculated using Algorithm 2. This number is the total number of TSs that are considered to estimate the error rate contribution of class $(a, b)$ TSs and is compared to the total number of TSs in that class. Table 4.2 shows that the PDTS extraction technique significantly reduces the number of 


\begin{tabular}{|c|c|c|c|c|c|c|}
\hline \multicolumn{2}{|r|}{ Constellation } & 8-PAM & 16-PAM & $32-\mathrm{PAM}$ & $64-\mathrm{PAM}$ & 128-PAM \\
\hline \multicolumn{2}{|r|}{ SNR values $(\mathrm{dB})$} & 121314 & 141516 & 192021 & 242526 & 272829 \\
\hline TS class $(8,2)$ & Number of PDTSs & 70 & 21 & 13 & 16 & 15 \\
\hline$\left|\mathcal{T}_{(8,2)}\right|=465$ & Error rate contribution (\%) & 879190 & 789193 & 737780 & 939695 & 808286 \\
\hline$J_{a b}=1$ & Number of required trials $\left(\times 10^{6}\right)$ & 3.6253 .813 .85 & 1.1351 .331 .345 & 4.154 .655 .25 & 3.4653 .12 .67 & 12.512 .511 .5 \\
\hline TS class $(10,2)$ & Number of PDTSs & 136 & 30 & 25 & 9 & 7 \\
\hline$\left|\mathcal{T}_{(10,2)}\right|=1395$ & Error rate contribution (\%) & 13910 & 2297 & 272320 & 745 & 201814 \\
\hline$J_{a b}=2$ & Number of required trials $\left(\times 10^{6}\right)$ & 8.217 .757 .43 & 3.283 .222 .29 & 16.917 .117 .2 & 4.3153 .613 .68 & 2.652 .652 .64 \\
\hline \multicolumn{2}{|c|}{ Simulation gain - $10 \log _{10}[\eta]$ - cf. (4.10) } & $-7.7-0.210 .1$ & -3.41 .910 .2 & -0.76 .513 .9 & 8.315 .624 & 0.5612 .7 \\
\hline
\end{tabular}

Table 4.2: The number of estimated TSs, the relative contribution of a given TS in the total estimated FER, and the total number of trials for the estimation of a TS class are provided for the estimated FERs in Fig. 4.3.

TS candidates. For example for TS class $(10,2)$, the $\left|\mathcal{T}_{(10,2)}\right|=1395$. Now, for $32-\mathrm{PAM}$ signalling at an SNR of $20 \mathrm{~dB}$ and with $\beta_{\mathcal{T}}^{\text {th }}=0.025$ the corresponding number of PDTSs is 25 and the total number of TSs with non-identical BL sets is $\sum_{j=1}^{J_{a b}} \sum_{t=}^{N_{a b, j}}\left|\mathcal{T}_{(a, b)}^{j, t}\right|=1171$. Hence, the number of TSs considered in our method is only $2.1 \%$ of all the TS within this class.

2. Effect of $\beta_{\mathcal{T}}^{\text {th }}$ on the estimation performance: The PDTS extraction technique is based on a prescribed threshold, $\beta_{\mathcal{T}}^{\text {th }}$, to select the TS candidates for which the estimation is performed. The value of $\beta_{\mathcal{T}}^{t h}$ provides a trade-off between the estimation accuracy and computational complexity; decreasing $\beta_{\mathcal{T}}^{\text {th }}$ improves estimation accuracy but increases the computational complexity. This trade-off is illustrated in Fig. 4.7 for the Tanner $(155,64)$ code with 128-PAM signalling. In this figure, we consider three values of $\beta_{\mathcal{T}}^{\text {th }}$, namely $0.125,0.05$, and 0 . From this figure it can be observed that by decreasing the value of $\beta_{\mathcal{T}}^{\text {th }}$, the estimation accuracy is improved. However, this improvement is relatively small. The marginal difference between the curves corresponding to $\beta_{\mathcal{T}}^{\text {th }}=0$ and the other two thresholds show that the PDTS extraction technique precisely selects the TS candidates that are more prone to errors. In other words, the error rate contribution of the TSs discarded by the PDTS is negligible.

To compare the computational complexity corresponding to each value of $\beta_{\mathcal{T}}^{\text {th }}$ in Table 4.3 we show the number of PDTSs and the total number of trials to 


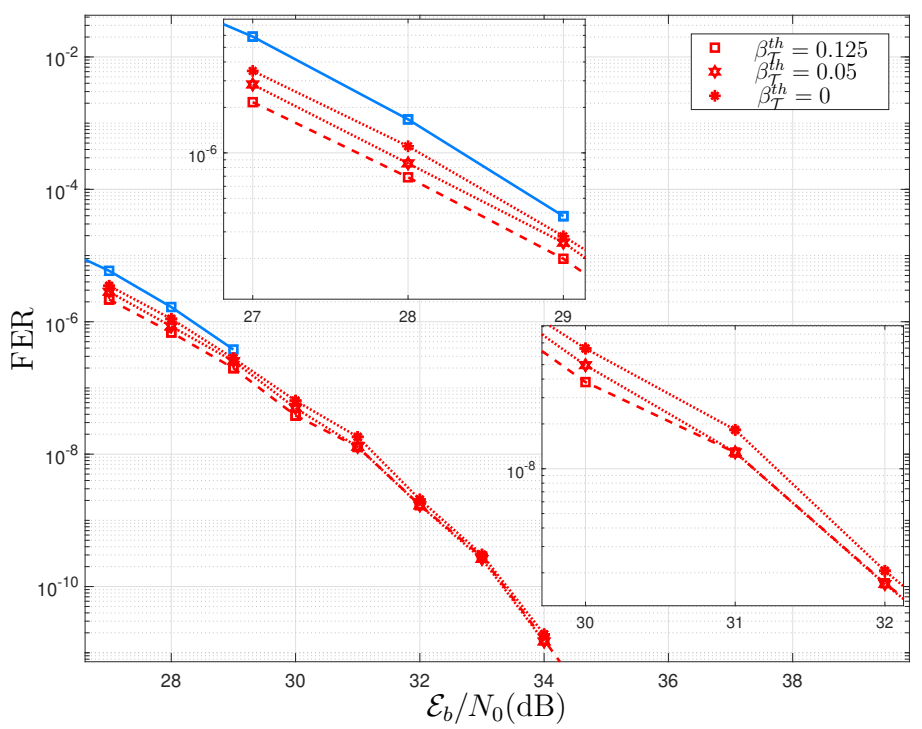

Figure 4.7: Effect of $\beta_{\mathcal{T}}^{\text {th }}$ on the performance of proposed MS-IS technique is illustrated by showing the estimated FER curves corresponding to three threshold values. The solid line and the dashed line show the FER curves corresponding to MC and MS-IS schemes, respectively.

estimate the EF that are made in each case at an SNR of $27 \mathrm{~dB}$. The last row of this table shows the estimated FERs. The FER corresponding to $\beta_{\mathcal{T}}^{\text {th }}=0$ is the most accurate since it corresponds to the cases when the MS-IS scheme considers the contributions of all the TSs. In other words, the PDTS scheme is not used. This table confirms that using the PDTS extraction technique yields a significant reduction in the number of required trials to estimate the error rate contributions. For instance, the number of trials corresponding to $\beta_{\mathcal{T}}^{t h}=0.125$ (the threshold initially chosen using Table 4.1 ) is almost $0.03 \%$ of the number of trials corresponding to the case when the PDTS extraction is not used, i.e., the case of $\beta_{\mathcal{T}}^{\text {th }}=0$.

3. Comparison between the time efficiency of MC and MS-IS: When MC simulation is used, the number of required trials for maintaining a constant estimation variance is inversely proportional to the FER, cf. (4.7), rendering it impractical for estimating the low FERs. In contrast with the MC simulation, the accuracy of MS-IS scheme does not depend on the FER. 


\begin{tabular}{|c|c|c|c|c|}
\hline & \multirow{3}{*}{$\begin{array}{c}\beta_{\mathcal{T}}^{\text {th }}=0.125 \\
15\end{array}$} & \multirow{2}{*}{$\beta_{\mathcal{T}}^{\text {th }}=0.05$} & \multirow{2}{*}{$\beta_{\mathcal{T}}^{\text {th }}=0$} \\
\hline & & & & \\
\hline TS Class & Number of PDTSs & & 19 & 393 \\
\hline$(8,2)$ & Number of Required Trials $\left(\times 10^{6}\right)$ & 12.5 & 17.8 & 389 \\
\hline TS Class & Number of PDTSs & 7 & 9 & 1291 \\
\hline$(10,2)$ & Number of Required Trials $\left(\times 10^{6}\right)$ & 2.65 & 3.75 & 52000 \\
\hline & Estimated FER & $2.16 \times 10^{-6}$ & $2.83 \times 10^{-6}$ & $3.48 \times 10^{-6}$ \\
\hline
\end{tabular}

Table 4.3: The number of PDTSs and the required trials for estimating the EF of Tanner $(155,64)$ code at an SNR of $27 \mathrm{~dB}$ for 128-PAM signalling is displayed. Three $\beta_{\mathcal{T}}^{\text {th }}$ values are considered.

As the SNR increases and we enter deeper into the EF region, the constant number of required trials of the MS-IS scheme gives it an important advantage over its MC counterpart. For example, from Table 4.2, the number of required trials to estimate the FER of the Tanner code with 32-PAM signalling at SNRs of $20 \mathrm{~dB}$ and $21 \mathrm{~dB}$ for the proposed MS-IS scheme is $2.17 \times 10^{7}$ and $2.24 \times 10^{7}$, whereas these numbers for the MC scheme are $1.21 \times 10^{8}$ and $8.1 \times$ $10^{8}$, respectively. This confirms that the time efficiency of the MS-IS scheme increases with the SNR.

We note that in comparing the time efficiency of MC and proposed MS-IS scheme the complexity of initial MC simulation to obtain the required TS classes, $\mathcal{B}$, is neglected since: 1) such simulation is performed once and the results are used for all the SNRs that the MS-IS scheme is applied in them. Hence such complexity is divided among all SNRs, 2) The complexity of MC simulation at early EF region is commonly much smaller than the one at higher SNRs. Hence, adding the computational complexity of initial MC simulation would result in small reduction of achieved simulation gains. In particular, if the average number of required trials for the initial MC simulation is denoted by $N_{e M C}$, the degradation in simulation gain by adding $N_{e M C}$ in the denominator of $(4.10)$ becomes $10 \log _{10}\left(1+\frac{N_{e M C}}{N_{I S}}\right) \mathrm{dB}$. As an example, in the case of Tanner code with 64-PAM signalling we set $N_{e M C}=\frac{8.34 \times 10^{5}}{3}=2.78 \times 10^{5}$ where $8.34 \times 10^{5}$ is the number of required trials at the first FER where the errors are dominated by TSs rather than being random and 3 is the number of SNR points, in which the MS-IS scheme is applied in Table 4.2. By using Table 4.2 
and the aforementioned formula, the degradation in the simulation gains due to the initial MC simulation will be $0.15,0.18$, and $0.19 \mathrm{~dB}$ for the SNRs 24, 25, and $26 \mathrm{~dB}$, respectively.

4. Number of required trials in the MS-IS scheme for different constellation sizes: Although the number of required trials increases with the size of the TS, this trend is not observed when the size of the constellation increases. In particular, the number of required trials to estimate the error rate of a given TS class may increase or decrease with the size of the constellation. This is because the BL set of a given TS depends on the constellation size, which implies that the parameters $P_{\mathcal{T}_{\max }}$ and $\beta_{\mathcal{T}}^{\text {th }}$ in Algorithm 2 will depend on the constellation size. The number of required trials to meet the accuracy threshold of the estimation will be lower for the $\mathrm{BL}$ sets with large $P_{\mathcal{T}_{\max }}$ (and vice-versa); however, by increasing the constellation size $P_{\mathcal{T}_{\max }}$ may increase or decrease. This nonmonotonic behavior results in the lack of certain trend in the number of required trials for the MS-IS scheme when the constellation size goes up. For example, for the case of the Tanner code in Table 4.2, the number of required trials to estimate the error rate of class $(8,2)$ is approximately $3.6 \times 10^{6}$ and $1.1 \times 10^{6}$ for the 8-PAM and the 16-PAM, respectively. This is a reduction of almost $60 \%$ in the number of required trials.

5. Number of PDTSs corresponding to a given TS class for different constellation sizes: From Table 4.2 it can be observed that in the majority of cases, for a given TS class the number of PDTSs decreases as the constellation size increases. To explain this behavior, we note that as the constellation size increases, the values of $\operatorname{Pr}(\mathrm{T}$-weak codeword) are distributed over a wider range, cf. (4.30). Hence, the number of BL sets with higher $\operatorname{Pr}(\mathrm{T}$-weak codeword) values decreases. For instance, the number of PDTSs for class $(10,2)$ TS is 136 for 8-PAM, 30 for 16-PAM, and 7 for 128-PAM. 


\section{Chapter 5}

\section{Error Floor Estimation of QAM-based LDPC Coded Modulation Systems}

In Chapter 4, we described the proposed scheme for estimating the EF of LDPC-CMSs using PAM signalling. However, state-of-the-art digital communication systems aim at exploiting bandwidth efficient signalling schemes. An example of such signalling schemes is the QAM one, that is widely used in the modern communication systems,

see; e.g., [4-6, 8]. Besides, QAM signalling is the dominant signalling method in the PtPMRLs $[8,43,84]$. In this chapter, we extend the proposed EF estimation scheme to LDPC-CMSs using QAM signalling. Furthermore, in this chapter, we describe a method to obtain the minimum number of required trials in the proposed MS-IS scheme. We also explain some minor modifications in the proposed MS-IS scheme for the cases when more than one TS VN is mapped to a given signal constellation. These points facilitates the time-efficient implementation of the proposed algorithms in Chapters 4, and 5.

\subsection{Required Modifications in the Proposed EF Estimation Scheme}

Towards extending the proposed EF estimation technique to QAM-based LDPCCMSs, we will use the fact that a QAM symbol can be decomposed into an in-phase and a quadrature component, each belonging to a PAM constellation.Hence, we apply the method proposed in Chapter 4 to the in-phase and the quadrature components 
of the received signals independently. Doing so requires modifying the transmit and receive sides of the system, as we elaborate below

\subsubsection{Labelling the BLs}

In Chapter 4, we considered Gray-labeled $M-\mathrm{PAM}$ constellation with $M=2^{m}$ symbols. We then assigned a BL of 1 to the MSB and a BL of $m$ to the LSB. The philosophy of this assignment is that the bits that receive more protection from the constellation are indexed with lower BLs. To follow a similar labelling scheme in a QAM constellation of size $2^{2 m}$, we treat the bits corresponding to the in-phase and the quadrature components independently. Let us assume that $s=s_{i}+j s_{q}$ is a QAM symbol where $s_{i}$ and $s_{q}$ are the in-phase and quadrature components, respectively. To apply the same BL assignment philosophy as in the PAM case, we label the bits corresponding to each component from 1 to $m$. Hence, while the number of bits mapped to each QAM symbol is $2 m$, there are only $m$ BLs involved in our calculations.

\subsubsection{Calculating the MS values}

In a $2^{2 m}$-QAM system, the noise samples corresponding to the transmitted symbols are complex values. Hence, the MS values can be added to the real, imaginary, or both components of the noise samples. To determine which component of a noise sample needs to be biased, we identify the TS VNs that are connected to the inphase components, and those that are connected to the quadrature components of the transmitted symbols. The TS VNs that are mapped to the in-phase and the quadrature components are treated as two hypothetical TSs in a PAM system with constellation size $2^{m}$. To elaborate, we will now explain the MS calculation procedure for a TS of size $a$ in a system using a $2^{2 m}$-QAM constellation.

For a TS, T, of size $a$, the set of VNs mapped to the in-phase and the quadrature components of the transmitted symbols are denoted by $\mathrm{T}_{i}$, and $\mathrm{T}_{q}$, respectively. Let $\left|\mathrm{T}_{i}\right|=a_{i}$, and $\left|\mathrm{T}_{q}\right|=a_{q}$, where $a_{i}+a_{q}=a$. Applying the MS calculation scheme of Section 4.3.2 to $\mathbf{T}_{i}$, we obtain $a_{i}$ MS values, $\left\{\mu_{\mathrm{T}_{i}}^{v}\right\}_{v=1}^{a_{i}}$. Using an analogous approach, we obtain $a_{q}$ MS values, $\left\{\mu_{\mathrm{T}_{q}}^{v}\right\}_{v=1}^{a_{q}}$ corresponding to $\mathrm{T}_{q}$. We now identify the indices of the noise samples to which the calculated MS values are added. To do so, we build 
the sets $\zeta_{\mathrm{T}_{i}}=\left\{\zeta_{\mathrm{T}_{i}}^{v}\right\}_{v=1}^{a_{i}}$ and $\zeta_{\mathrm{T}_{q}}=\left\{\zeta_{\mathrm{T}_{q}}^{v}\right\}_{v=1}^{a_{q}}$, as per the definition in Section 4.1.2. The elements of $\zeta_{\mathbf{T}_{i}}$ determine the indices of the noise samples, which are biased by adding the corresponding elements from $\left\{\mu_{\mathrm{T}_{i}}^{v}\right\}_{v=1}^{a_{i}}$ to their real part. Similarly, the noise samples indexed with the elements of $\zeta_{T_{q}}$ are biased by adding the corresponding MS values in $\left\{\mu_{\mathrm{T}_{q}}^{v}\right\}_{v=1}^{a_{q}}$ to their imaginary part.

As an illustrative example, consider the TS $\mathrm{T}=\{396,467,548,570\}$ (cf. Example 2) when a 64-QAM constellation is used. In this case, $\mathrm{T}_{i}=\{396,548,570\}$, $\mathrm{T}_{q}=\{467\}, \zeta_{\mathbf{T}_{i}}=\{66,92,95\}$, and $\zeta_{\mathbf{T}_{q}}=\{78\}$. We consider $\mathbf{T}_{i}$ and an 8-PAM constellation, and use the scheme described in Section 4.3.2, to obtain three MS values, $\left\{\mu_{\mathrm{T}_{i}}^{v}\right\}_{v=1}^{3}$. Similarly, $\mathbf{T}_{q}$ is used to calculate a single MS value, $\mu_{\mathrm{T}_{q}}^{1}$, to be added to the imaginary part of the noise sample with index 78 .

\subsubsection{Calculating the weight function}

To obtain the weight function in a QAM-based system, the joint PDFs of the biased and unbiased received signals should be calculated. To do so, we use the following observation: For a given codeword, $\mathcal{C}$, the joint $\mathrm{PDF}$ of the received signals is identical to the joint PDF of their real and imaginary components. For instance, the joint PDF of the unbiased received signals $f_{\boldsymbol{R} \mid \mathcal{C}}(\boldsymbol{r})=f_{\boldsymbol{R}_{i} \mid \mathcal{C}}\left(\boldsymbol{r}_{i}\right) f_{\boldsymbol{R}_{q} \mid \mathcal{C}}\left(\boldsymbol{r}_{q}\right)$, where $f_{\boldsymbol{R}_{i} \mid \mathcal{C}}\left(\boldsymbol{r}_{i}\right)$, and $f_{\boldsymbol{R}_{q} \mid \mathcal{C}}\left(\boldsymbol{r}_{q}\right)$ are the joint PDFs of the real and the imaginary parts of the received signals, respectively. It can be readily seen that $f_{\boldsymbol{R}_{i} \mathcal{C}}\left(\boldsymbol{r}_{i}\right)=\prod_{k=1}^{N_{s}} f_{R_{i, k}}\left(r_{i, k}\right)$, where the subscript $i, k$ denotes the real part of the $k$-th element, and $R_{i, k} \sim \mathcal{N}\left(s_{i, k}, \frac{N_{0}}{2}\right)$. Similarly, $f_{\boldsymbol{R}_{q} \mid \mathcal{C}}\left(\boldsymbol{r}_{q}\right)=\prod_{k=1}^{N_{s}} f_{R_{q, k}}\left(r_{q, k}\right)$, where the subscript $q, k$ denotes the imaginary part of the $k$-th element, and $R_{q, k} \sim \mathcal{N}\left(s_{q, k}, \frac{N_{0}}{2}\right)$. The joint PDF of the biased received signals $f_{\boldsymbol{Z} \mid \mathcal{C}}(\boldsymbol{z})=f_{\boldsymbol{Z}_{i} \mid \mathcal{C}}\left(\boldsymbol{z}_{i}\right) f_{\boldsymbol{Z}_{q} \mid \mathcal{C}}\left(\boldsymbol{z}_{q}\right)$, where $f_{\boldsymbol{Z}_{i} \mid \mathcal{C}}\left(\boldsymbol{z}_{i}\right)$ is obtained by replacing $\left\{\mu_{\boldsymbol{T}_{i}}^{v}\right\}_{v=1}^{a_{i}}$, and $\zeta_{\mathbf{T}_{i}}$ in (4.17). Hence, for $f_{\boldsymbol{Z}_{i} \mid \mathcal{C}}\left(\boldsymbol{z}_{i}\right)$ we have:

$$
f_{\boldsymbol{Z}_{i} \mid \mathcal{C}}\left(\boldsymbol{z}_{i}\right)=\left(\prod_{k \in \zeta_{\mathrm{T}_{i}}} f_{R_{i, k}}\left(z_{i, k}-\mu_{\mathbf{T}_{i}}^{v(k)}\right)\right)\left(\prod_{k \in\left\{1, \ldots, N_{s}\right\} \backslash \zeta_{\mathrm{T}_{i}}} f_{R_{i, k}}\left(z_{i, k}\right)\right) .
$$


In a similar fashion, $f_{\boldsymbol{Z}_{q} \mid \mathcal{C}}\left(\boldsymbol{z}_{q}\right)$ is calculated by replacing $\left\{\mu_{\mathrm{T}_{q}}^{v}\right\}_{v=1}^{a_{q}}$, and $\zeta_{\boldsymbol{T}_{q}}$ in (4.17) to obtain $f_{\boldsymbol{Z}_{q} \mid \mathcal{C}}\left(\boldsymbol{z}_{q}\right)$ as follows:

$$
f_{\boldsymbol{Z}_{q} \mid \mathcal{C}}\left(\boldsymbol{z}_{q}\right)=\left(\prod_{k \in \zeta_{T_{q}}} f_{R_{q, k}}\left(z_{q, k}-\mu_{\mathrm{T}_{q}}^{v(k)}\right)\right)\left(\prod_{k \in\left\{1, \ldots, N_{s}\right\} \backslash \zeta_{\mathrm{T}_{q}}} f_{R_{q, k}}\left(z_{q, k}\right)\right) .
$$

The weight function in a QAM-based system, is obtained by taking the ratio of $f_{\boldsymbol{R} \mid \mathcal{C}}(\boldsymbol{r})$, and $f_{\boldsymbol{Z} \mid \mathcal{C}}(\boldsymbol{z})$. By using (5.1) and (5.2), we have:

$$
w_{\mathcal{C}}(\boldsymbol{z})=\frac{f_{\boldsymbol{R}_{i} \mid \mathcal{C}}\left(\boldsymbol{r}_{i}\right) f_{\boldsymbol{R}_{q} \mid \mathcal{C}}\left(\boldsymbol{r}_{q}\right)}{f_{\boldsymbol{Z}_{i} \mid \mathcal{C}}\left(\boldsymbol{z}_{i}\right) f_{\boldsymbol{Z}_{q} \mid \mathcal{C}}\left(\boldsymbol{z}_{q}\right)}=\prod_{k \in \zeta_{T_{i, k}}} \frac{f_{R_{i, k}}\left(z_{i, k}\right)}{f_{R_{i, k}}\left(z_{i, k}-\mu_{\mathrm{T}_{i}}^{v(k)}\right)} \prod_{k \in \zeta_{\boldsymbol{T}_{q}}} \frac{f_{R_{q, k}}\left(z_{q, k}\right)}{f_{R_{q, k}}\left(z_{q, k}-\mu_{\mathbf{T}_{q}}^{v(k)}\right)}
$$

To estimate $\operatorname{Pr}(\mathcal{D F}, T)$ for an LDPC-CMS using QAM signalling, we use (4.20), with $w_{\mathcal{C}}^{(i)}(\boldsymbol{z}(\mathcal{C}, W))$ being calculated from (5.3). The rate of the decoding failure in the EF region is estimated using (4.16), where $\mathcal{T}_{(a, b)}^{j, t}$ and $N_{a b, j}$ are obtained as in Section 4.3 using the BL sets corresponding to the QAM constellation under consideration.

\subsection{Simulation Results}

In this section, we apply the proposed EF estimation schemes to the LDPC-CMSs using QAM signalling. Similar simulation parameters to what were used in Section 4.5 are considered here. Regarding the QAM constellation size, we consider $M$-QAM signalling, where $M=2^{2 m}, m \in\{2, \ldots, 5\}$.

We begin by the Tanner $(155,64)$ LDPC code. For this code, we have 2 dominant TS classes, namely $(8,2)$ and $(10,2)$ [40]. The MC simulation verifies that both classes are present when the QAM constellations are used. Thus, we set $a_{\max }=10$ and $b_{\max }=2$. Figure 5.1 shows the MC and the MS-IS FER estimation results when the QAM signalling scheme is used. It can be seen that the MS-IS estimation and the MC simulation curves give similar results. This shows that the proposed MS-IS estimation scheme can effectively estimate the FER in the EF region. 


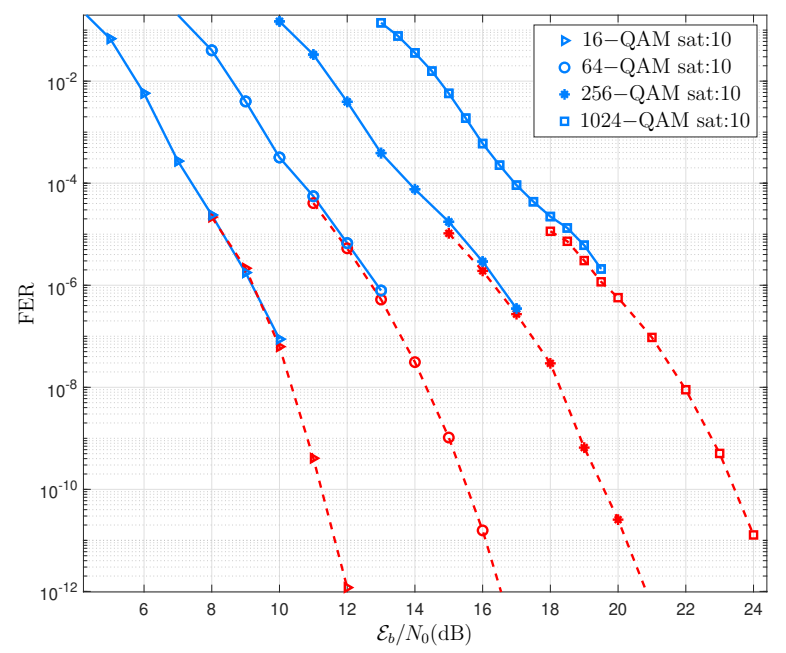

Figure 5.1: The MC (solid-line) and the MS-IS (dashed-line) simulation results for the Tanner $(155,64)$ LDPC code with $M$-QAM signalling.

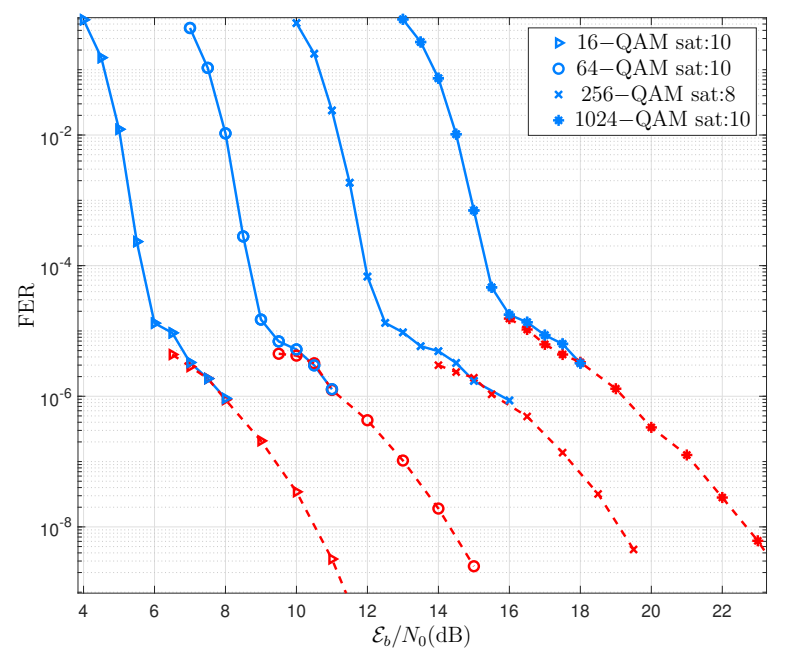

Figure 5.2: The MC (solid-line) and the MS-IS (dashed-line) simulation results for the MacKay $(1008,504)$ LDPC code with $M$-QAM signalling.

The MC and MS-IS FER estimation results for the QAM-based LDPC-CMS using MacKay $(1008,504)$ LDPC code are displayed in Fig. 5.2. The MC simulation at early EF region demonstrates that the class $(8,2)$ is the largest size TS class with non-negligible FER. Hence, we set $a_{\max }=8$ and $b_{\max }=2$ to estimate the FER. Here we should consider 3 TS classes, namely $(4,2),(6,2)$, and $(8,2)$. It can be seen that both methods yield similar FER estimate.

For the WiMAX LDPC codes the FER curves are shown in Figs. 5.3 and 5.4 


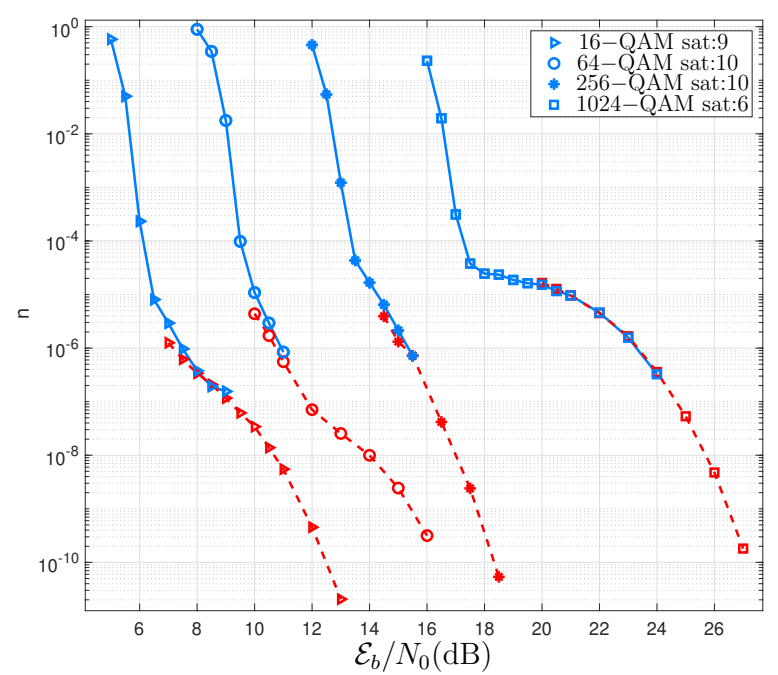

Figure 5.3: The MC (solid-line) and the MS-IS (dashed-line) simulation results for the WiMAX1 $(2016,1344)$ LDPC code with $M$-QAM signalling.

to illustrate the effectiveness of the proposed technique for estimating the EF when using the irregular LDPC codes with QAM signalling. From MC simulation at early EF region, for the WiMAX1 $(2016,1344)$, we obtain $a_{\max }=9$ and $b_{\max }=2$. By using these values, we consider 9 TS classes, namely, $(8,1),(9,1),(3,2),(4,2),(5,2),(6,2)$, $(7,2),(8,2)$, and $(9,2)[42]$, to estimate the EF. For the WiMAX2 $(1152,960)$ LDPC code, MC simulation yields $a_{\max }=7$ and $b_{\max }=2$. Hence, 9 TS classes, namely, $(3,1),(4,1),(5,1),(6,1),(7,1),(4,2),(5,2),(6,2)$, and $(7,2)[42]$, are required to estimate the EF. The FER results for MC and the proposed MS-IS scheme are shown in Figs. 5.3, and 5.4, and confirm that both schemes result in similar FER curves. Similar to the case of LDPC-CMSs using PAM signalling, in the QAM-based LDPCCMSs, the computational complexity of estimating the EF can be further reduced by invoking the QC structure of WiMAX code. The error rate contributions of the TS classes used in the EF estimation of QAM-based LDPC-CMSs in Figs. 5.1- 5.4 are displayed in Tables C.1- C.4 in Appendix C.1. 


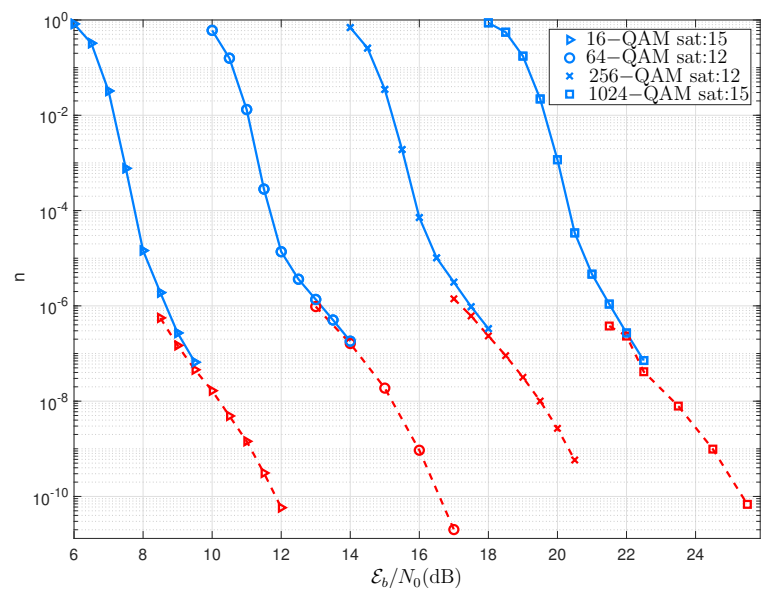

Figure 5.4: The MC (solid-line) and the MS-IS (dashed-line) simulation results for the WiMAX2 $(1152,960)$ LDPC code with $M$-QAM signalling.

\subsection{Discussions on Implementing the Proposed EF Estimation Algorithm}

In Chapter 4 and the first two sections of this chapter, we presented our proposed EF estimation scheme applicable to LDPC-CMSs using PAM and QAM signalling. This presentation is sufficient for comprehending the ideas behind our estimation algorithm, and for implementing the estimation scheme. The purpose of this section, however, is to provide further details on the implementation process. By using the discussions in this section, the interested reader finds the implementation of the proposed algorithm more convenient. In brief, this section aims at facilitating the application of the proposed methods and algorithms in practice.

\subsubsection{Number of Required Trials}

As it was explained in Chapter 4, the number of required trials to estimate the error rate of a given TS can be determined by setting the reliability threshold. This approach does not offer any insight about the relative number of required trials for estimating the error rate of various TSs. This is because one needs to conclude the estimation process to know the number of required trials. In practice, we may need to estimate the error rate of a large number of TSs from different classes. To implement the estimation method more efficiently, it is desirable to have an initial evaluation of 
the number of required trials to estimate the error rate of a given TS. Towards this end, in the following discussion, we calculate the minimum number of required trials to estimate the error rate of a given TS. Doing so determines the minimum number of times that one needs to simulate the transceiver architecture to estimate the error rate of a given TS.

To calculate the minimum number of required trials to estimate the error rate of a given TS, we use the probability of T-weak codeword. In the forthcoming discussion, $\operatorname{Pr}\left(\mathrm{T}\right.$-weak codeword) is denoted by $\mathrm{P}_{\mathcal{W}_{\mathrm{T}}}$ for notation brevity. Note that T-weak codewords result in large weight function values. To obtain a meaningful estimate of the error rate, sufficient number of those large weights are required. Besides, $\mathrm{P}_{\mathcal{W}_{\mathrm{T}}}$ indicates the probability of generating a large weight for an individual trial. We note that each trial is equivalent to generating one codeword. We can model the codeword generation as a sequence of Bernoulli trials, and assume $\mathrm{P}_{\mathcal{W}_{\top}}$ as the probability of success. When $V$ codewords are generated, the probability of existing at least $v$ T-weak codewords among them can be calculated as follows:

$$
\mathcal{P}(\text { at least } v \mathrm{~T} \text {-weak codewords among } V)=1-\left(\begin{array}{c}
V \\
v-1
\end{array}\right)\left(\mathrm{P}_{\mathcal{W}_{\mathrm{T}}}\right)^{v-1}\left(1-\mathrm{P}_{\mathcal{W}_{\mathrm{T}}}\right)^{V-v+1} \text {. }
$$

The goal is to have at least $v \mathrm{~T}$-weak codewords, which result in $v$ large value weights. We consider $v$ and the probability at the LHS of (5.4) as the design parameters. This will not cause any inaccuracies since the goal is to find an initial value for the required number of trials and then continuing the experiment until we reach to the reliability threshold which is set for the estimation. By replacing (4.30) in (5.4) and choosing $v$ and setting a small number, e.g. $10^{-15}$, to the LHS of (5.4), we can numerically solve (5.4) for $V$. The calculated $V$ is the minimum number of required codewords such that $v$ of them are $\mathrm{T}$-weak codewords. This number is denoted by $V_{\min }$.

In the above discussion, there is an implicit assumption that for a T-weak codeword, the parameter $\mathbb{I}_{\boldsymbol{R}_{E}}^{(i)}(\boldsymbol{z})$ in (4.20) is equal to one. The importance of the indicator function lies in the fact that when $\mathbb{I}_{\boldsymbol{R}_{E}}^{(i)}(\boldsymbol{z})=0$, the weight function value is irrelevant in the summation (it will be multiplied by 0 ). By letting $\mathbb{I}_{\boldsymbol{R}_{E}}^{(i)}(\boldsymbol{z}) \equiv 1$ we are assuming after the biasing is carried out, the probability of $\mathcal{D} \mathcal{F}$ on $\mathrm{T}$ is equal to 1. This is not always a valid assumption since there are cases, in which a larger (or 
smaller) size TS appears during the simulation of the target TS. We obviously ignore those cases (put $\mathbb{I}_{\boldsymbol{R}_{E}}^{(i)}(\boldsymbol{z})=0$ for those trials). To alleviate this difficulty, we note that extensive simulation results show that $\operatorname{Pr}\left(\mathbb{I}_{\boldsymbol{R}_{E}}^{(i)}(\boldsymbol{z})=1\right)$ is constantly in the vicinity of 1 , and will not become smaller than 0.1 with high probability. To take this point into consideration, we multiply $V_{\text {min }}$ by $\frac{1}{\mathcal{P}\left(\mathbb{I}_{\boldsymbol{R}_{E}}^{(i)}(\boldsymbol{z})=1\right)}$. Since $\mathcal{P}\left(\mathbb{I}_{\boldsymbol{R}_{E}}^{(i)}(\boldsymbol{z})=1\right)$ depends on many parameters and cannot be determined a periori, we consider using a lower bound, e.g. 0.1. Upon determining the minimum number of the required codewords, $V_{\text {min }}$, we use Algorithm 3 to determine $N_{I S}$.

Algorithm 3 Finding the number of required trials, $N_{I S}$, for the estimation of $\mathcal{P}(\mathcal{D F}, \mathrm{T})$.

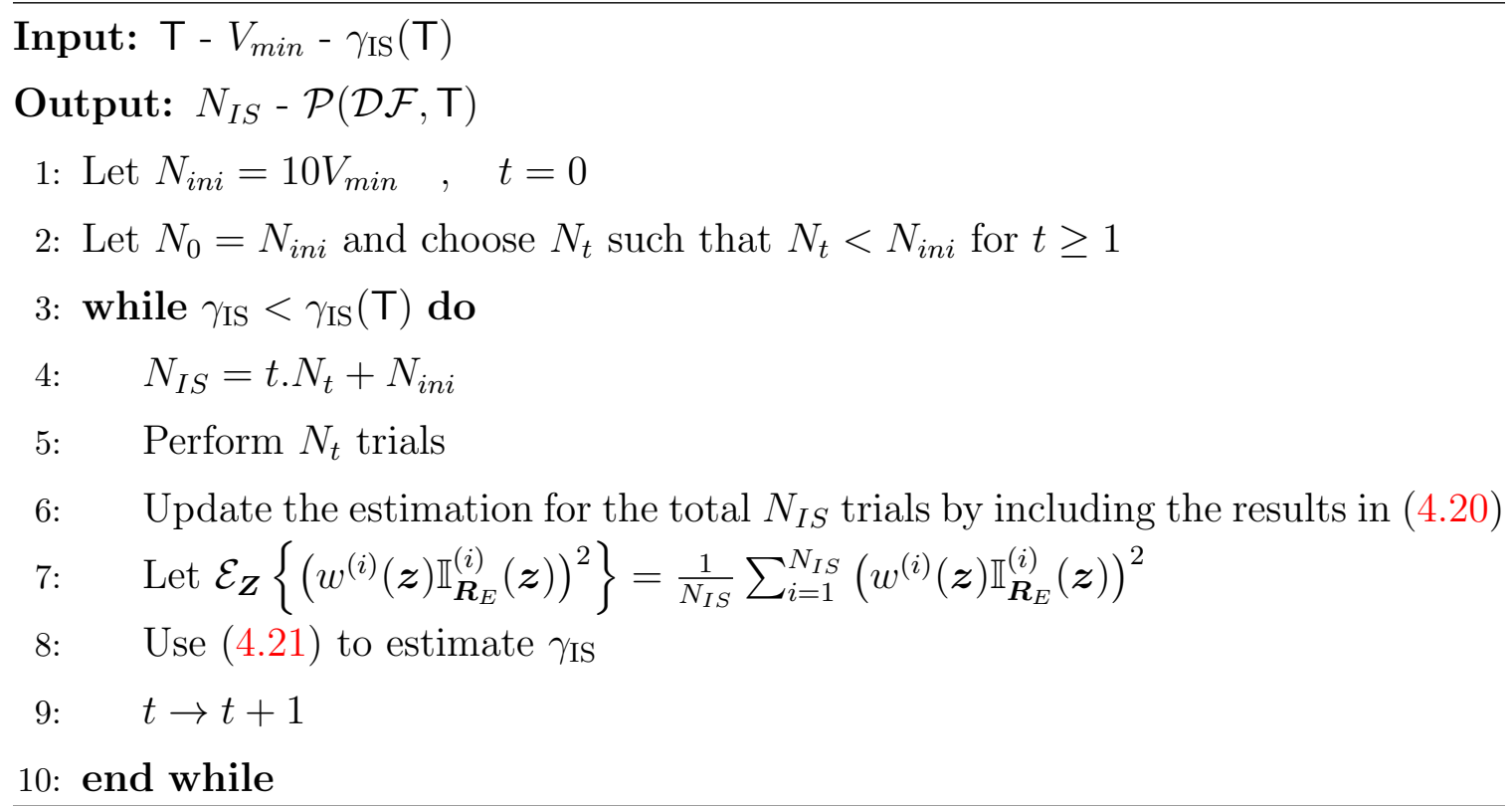

\subsubsection{Multiple TS VNs Mapped to One Constellation Point}

Up to now, we assumed no more than one VN of a TS is mapped to a given symbol. Although valid in the majority of cases, this assumption does not always hold true. When the LDPC code has short length (e.g., $N<1000$ ), and the modulation size is large (e.g., when each symbol carries $m \geq 4$ bits), the number of cases which violate the aforementioned assumption becomes non-negligible. Hence, the estimation scheme should be modified accordingly. In the following, we explain these modifications. Note that the following modifications are applicable in either of these two 
situations: 1) In a PAM-based system, where more than one VN of a TS is mapped to a given symbol, and 2) In a QAM-based system, where more than one VN of a TS is mapped to one or both components of a given symbol.

We begin by modifying the MS calculation. Note that here, the number of symbols that the TS VNs are mapped to them is not $a$. Instead, this number reduces to $\hat{a}$, where $\hat{a}<a$. This change, initially, reflects on the step 1 in Algorithm 1, where the loop will go from 1 to $\hat{a}$ (rather than 1 to $a$ ). Furthermore, in Algorithm 1, we modify the steps 4 and 5 . To do so, we denote the number of target bits in symbol $s_{T}^{v}$ with $\hat{m}$, where $1<\hat{m} \leq m$. Also, the vector of bits that are mapped to symbol $s_{T}^{v}$, and its subset of corresponding $\hat{m}$ target bits are denoted by $\boldsymbol{b}_{s_{T}^{v}}$ and $\hat{\boldsymbol{b}}_{s_{T}^{v}}$, respectively. To calculate the MS value corresponding to $s_{T}^{v}$, we make the following changes in Algorithm 1:

- In Step 4, flip $\hat{m}$ bits in $\hat{\boldsymbol{b}}_{s_{T}^{v}}$ rather than one target bit in $\boldsymbol{b}_{s_{T}^{v}}$.

- In Step 5, replace $\hat{m}$ target bits in $\hat{\boldsymbol{b}}_{s_{T}^{v}}$ rather than one target bit in $\boldsymbol{b}_{s_{T}^{v}}$.

Upon calculating the MS vector, the MS values are replaced in the biased PDF. Apart from the MS values, the only difference in the biased PDF is the number of terms. In this case, by using the definition in Section 4.1.2, we have $\zeta_{\mathrm{T}}=\left\{\zeta_{\mathrm{T}}^{v}\right\}_{v=1}^{\hat{a}}$. Hence, there exist $\hat{a}$ terms in the multiplication in the left parentheses in (4.17). Same applies to number of terms in the weight function formula in (4.18), and any formula, which includes the set $\zeta_{\mathrm{T}}$.

One characteristic of the cases where there exists more than one target bit in a symbol is the large MS values. This is because the chances that the target bits are in the BLs which do not differ in them with the adjacent symbols become higher. In other words, to locate a symbol that differs from the initial one in all the target bits, we should move to areas of the constellation far from the immediate neighbors of the unbiased symbol. The greater the MS becomes the smaller the weight function will be. The small weight functions result in the relatively smaller estimated values. This gives that we expect the TS to have a smaller error rates in these cases. 


\section{Chapter 6}

\section{Conclusion and Future Work}

In this thesis, we addressed two problems in the PHY layer of PtPMRLs. These issues are of great importance in modern microwave backhaul systems. To the best of author's knowledge, this work is the first to address these issues in the LDPC-CMSs used in PtPMRLs. It is worth noting that in addition to the contributions presented in the previous chapters, we proposed a novel LLR calculation in [34]. This scheme is integrated into the chipset of one of the PtP backhaul products manufactured by Huawei Technologies. Hence, the effectiveness of our proposed idea is verified on the FPGA, ASIC, and the product level. In the following, we briefly summarize the ideas proposed in this thesis. Subsequently, some ideas for extending the current research in further directions are provided.

\subsection{Thesis Contributions}

In Chapter 2 the required background for presenting the thesis as a self-contained document is provided. This chapter increases the accessibility of the thesis and makes it readable for wider audiences. PtPMRLs, the concept of PN, PH, the LDPC codes, and LDPC-CMSs were briefly introduced in Chapter 2.

In Chapter 3, we proposed a two-stage PH mitigation scheme for high order CMSs in PtPMRLs. The PH is modeled as a sudden change in the phase of the received signals, which happens at a random location and with a random magnitude. Using this model, we designed an NP-BHT to detect the PH when the PSAM PN mitigation scheme is applied. The optimal threshold for detecting the PH was 
analytically derived. The proposed PH detection scheme features low computational complexity and requires no extra pilot overhead. Upon detecting the $\mathrm{PH}$, our proposed $\mathrm{PH}$ correction scheme is triggered. An ML estimator is used to detect the $\mathrm{PH}$ location, and the $\mathrm{PH}$ magnitude. The estimated location and magnitude of $\mathrm{PH}$ along with the extrapolated $\mathrm{PN}$ from neighboring PSs are used to jointly remove the $\mathrm{PN}$ and the $\mathrm{PH}$ from the affected signals. By applying the proposed $\mathrm{PH}$ correction scheme, the number of $\mathrm{PH}$ affected signals are significantly reduced and the imminent decoder failure due to the $\mathrm{PH}$ is avoided.

The results in Chapter 3 are presented in the following articles:

- P.Neshaastegaran, M.Jian, 'DETECTION AND MITIGATION oF OSCILLATOR PHASE HIT' U.S. Patent Application\# 16/811,335.

- P.Neshaastegaran, M.Jian, 'A Double-Stage Phase Hit Mitigation Scheme In Microwave Backhaul Links,' in 2020 IEEE Latin-American Conference on Communications (LATINCOM), Santo Domingo, 2020

In Chapter 4, we developed a novel EF estimation scheme for high-order LDPC CMSs using PAM signalling. The proposed method is based on the importance sampling approach. In particular, an MS-IS scheme is designed to estimate the error rate contribution of each TS class. We show that; for non-binary signalling, the contribution of TSs within a given class are different. To exploit this property we developed a PDTS extraction technique to obtain the list of TSs with non-negligible contribution in the EF. This technique along with the proposed MS-IS scheme enables EF estimation of high order LDPC-CMSs that are significantly more time efficient than existing MC simulation-based schemes.

In Chapter 5, we extended the proposed EF estimation to LDPC-CMSs using QAM signalling. To do so, we began by modifying the labeling of BLs. Subsequently, an MS calculation method was proposed for the QAM-based LDPC-CMSs. Finally, the weight function was re-formulated for these systems. Simulation results confirm the effectiveness of proposed EF estimation scheme for teh QAM-LDPC coded systems.

The results in Chapters 4, and 5 are presented in the following article: 
- P. Neshaastegaran, A. H. Banihashemi, and R. H. Gohary, 'Error Floor Estimation of High-order LDPC Coded Modulation Systems Using Importance Sampling,' in IEEE Transactions on Communications, doi: 10.1109/TCOMM.2021.3057625.

\subsection{Future Research Directions}

The topics studied in this thesis can be extended in several ways. In the following, we elaborate on potential extensions.

- As it was mentioned in Chapter 1, it is anticipated that in future mobile communication infrastructure, the access and backhaul layers operate in a joint manner. This led to the idea of integrated access and backhaul, which is currently under study for Beyond $5 \mathrm{G}$ standardization. In view of such changes, the backhaul links are anticipated to use multi-carrier modulations, e.g., the orthogonal frequency division multiplexing (OFDM). PN mitigation in OFDM systems is a broad research topic which is substantially different from its singlecarrier counterpart. Similarly, the characterization of the RPN in the OFDM systems is completely different from the single-carrier scenario. Hence, as a future research direction, the LLR calculation in the presence of RPN can be studied for OFDM-based systems. Similar studies may be done for the PH mitigation scheme proposed in Chapter 3. Considering that the proposed PH mitigation scheme was built upon the PSAM PN mitigation approach, the extension of PH mitigation to OFDM-based systems will be non-trivial and worth further investigation.

- Further study of the PH mitigation scheme proposed in Chapter 3 can improve the detection and correction performance. For instance, the deficiency of the correction scheme when the hit magnitude is identical to the symmetry angle of the constellation can be considered as a future research direction.

- The EF estimation proposed in Chapters 4, and 5 is designed for the binary LDPC codes. The extension of proposed EF estimation to non-binary LDPC 
codes is a daunting task and an interesting research topic. It requires extension of TS search algorithm to non-binary codes alongside re-formulation of MS-IS scheme by calculating new MS values and deriving new biased PDFs and weight functions.

- As it was explained in Chapter 2, the PtPMRLs can be modeled using AWGN channel. Accordingly, the proposed EF estimation in Chapters 4, and 5, was developed based on the AWGN channel model. In fact, majority of literature, which discuss the EF of LDPC codes, assume the channel to be AWGN. However, in many practical scenarios, e.g., in mobile access layer, or optical communication links, there exist other influential parameters that affect the transmitted signals. In these systems, the effective channel cannot be modeled as AWGN. Extension of EF estimation in the systems that cannot be modeled by AWGN channel, is an interesting research direction. The complexity of such extension depends on the alternative channel model. For instance, access layer of mobile communication is commonly modeled using Rayleigh fading channel. The presence of fading coefficient adds an extra dimension to the signal model of LDPC-CMS. In this case, initially, the feasibility of using the MS-IS as the preferred IS scheme to induce more frequent error events should be verified. Subsequently, the MS calculation method should be modified based on the existence of two parameters (fading coefficient and additive noise) rather than a single parameter considered in this thesis. Moreover, the IS formulation needs to be modified accordingly. The aforementioned steps require extensive investigation and analysis, and would make an interesting research topic.

- EF estimation in LDPC-CMSs is closely related to the underlying constellation and bit labeling. The proposed EF estimation in Chapters 4, and 5 was built on the assumption of using PAM/QAM constellations that use Gray labeling. Recently, more sophisticated constellations and labeling schemes are considered in many practical systems, e.g., circular QAM, amplitude-phase-shift keying, and non-uniform constellations. In LDPC-CMSs using these alternative constellations and labeling, assessing the EF requires re-visiting the formulation of proposed EF estimation scheme. Specifically, the MS calculation method should 
be modified to be applicable in these systems. Extending the proposed EF estimation to constellations and labeling schemes other than what were discussed in Chapters 4, and 5, is an interesting research topic for the future. 


\section{List of References}

[1] T. S. Rappaport, S. Sun, R. Mayzus, H. Zhao, Y. Azar, K. Wang, G. N. Wong, J. K. Schulz, M. Samimi, and F. Gutierrez, "Millimeter wave mobile communications for 5G cellular: It will work!," IEEE Access, vol. 1, pp. 335-349, 2013.

[2] F. Boccardi, R. W. Heath, A. Lozano, T. L. Marzetta, and P. Popovski, "Five disruptive technology directions for 5G," IEEE Commun. Mag., vol. 52, no. 2, pp. 74-80, 2014.

[3] R. W. Heath, N. González-Prelcic, S. Rangan, W. Roh, and A. M. Sayeed, "An overview of signal processing techniques for millimeter wave MIMO systems," IEEE J. Select. Topics Signal Processing, vol. 10, no. 3, pp. 436-453, 2016.

[4] A. Gupta and R. K. Jha, "A survey of 5G network: Architecture and emerging technologies," IEEE Access, vol. 3, pp. 1206-1232, 2015.

[5] M. Jaber, M. A. Imran, R. Tafazolli, and A. Tukmanov, "5G backhaul challenges and emerging research directions: A survey," IEEE Access, vol. 4, pp. 1743-1766, 2016.

[6] T. Inoue, "5G NR release 16 and millimeter wave integrated access and backhaul," in 2020 IEEE Radio and Wireless Symposium (RWS), pp. 56-59, 2020.

[7] X. Huang, Y. J. Guo, A. Zhang, and V. Dyadyuk, "A multi-gigabit microwave backhaul," IEEE Commun. Mag., vol. 50, pp. 122-129, March 2012.

[8] Fixed Radio Systems; Characteristics and requirements for point-to-point equipment and antennas; Part 2: Digital systems operating in frequency bands from 1 GHz to 86 GHz. ETSI Standard EN 302 217-2 V3.1.1.

[9] E. Cetin, I. Kale, and R. C. S. Morling, "Living and dealing with RF impairments in communication transceivers," in 2007 IEEE International Symposium on Circuits and Systems, pp. 21-24, 2007.

[10] S. Mirabbasi and K. Martin, "Classical and modern receiver architectures," IEEE Communications Magazine, vol. 38, no. 11, pp. 132-139, 2000.

[11] R. M. Rao and B. Daneshrad, "Analog impairments in mimo-ofdm systems," IEEE Transactions on Wireless Communications, vol. 5, no. 12, pp. 3382-3387, 2006. 
[12] G. Boiocchi, P. Di Prisco, A. Lahrech, P. Lopez, M. Moretto, and P. Volpato, "Next-generation microwave packet radio: Characteristics and evolution areas to support new scenarios in wireless backhauling," Bell Labs Technical Journal, vol. 18, no. 2, pp. 143-157, 2013.

[13] M. Valkama, A. Springer, and G. Hueber, "Digital signal processing for reducing the effects of RF imperfections in radio devices - an overview," in Proceedings of 2010 IEEE International Symposium on Circuits and Systems, pp. 813-816, 2010.

[14] N. Kamiya and E. Sasaki, "Pilot-symbol assisted and code-aided phase error estimation for high-order QAM transmission," IEEE Transactions on Communications, vol. 61, pp. 4369-4380, October 2013.

[15] E. Ip and J. M. Kahn, "Feedforward carrier recovery for coherent optical communications," Journal of Lightwave Technology, vol. 25, pp. 2675-2692, Sept. 2007.

[16] E. Boch, "High-capacity ethernet backhaul radio systems for advanced mobile data networks," IEEE Microwave Magazine, vol. 10, no. 5, pp. 108-114, 2009.

[17] B. Zheng, L. Deng, M. Sawahashi, and N. Kamiya, "High-order circular QAM constellation with high LDPC coding rate for phase noise channels," in 2017 20th International Symposium on Wireless Personal Multimedia Communications (WPMC), pp. 196-201, 2017.

[18] G. L. Tan and N. G. Kingsbury, "Performance degradation of digital modulation schemes caused by random-walk phase noise," in IEEE International Conference on Communications, World Prosperity Through Communications,, pp. 16651669 vol.3, 1989.

[19] U. L. Rohde and A. K. Poddar, "Distributed coupled resonator (DCR) oscillators/VCOs limit phase noise and phase hits," in 2006 IEEE Radio and Wireless Symposium, pp. 447-450, Oct 2006.

[20] Y. Shen, L. Villeneuve, and N. Hassaine, "Dual loop push-push synthesizer for high modulation digital radios at 38ghz," in 2007 Asia-Pacific Microwave Conference, pp. 1-4, Dec 2007.

[21] Y. Shen, L. Villeneuve, and N. Hassaine, "High susceptibility of micro-phonic and phase-hit dual loop push-push synthesizer design for high modulation microwave digital radios," in 2006 Asia-Pacific Microwave Conference, pp. 516-519, Dec 2006.

[22] M. Jang, H. Lee, S. Kim, S. Myung, H. Jeong, and J. Kim, "Design of LDPC coded BICM in DVB broadcasting systems with block permutations," IEEE Trans. Broadcasting, vol. 61, pp. 327-333, June 2015. 
[23] S. Y. Le Goff, "Signal constellations for bit-interleaved coded modulation," IEEE Trans. Inf. Theory, vol. 49, pp. 307-313, Jan 2003.

[24] L. Michael and D. Gómez-Barquero, "Bit-Interleaved Coded Modulation (BICM) for ATSC 3.0," IEEE Trans. Broadcasting, vol. 62, pp. 181-188, March 2016.

[25] T. Richardson, "Error floors of LDPC codes," in Proc. Allerton Conf. Commun, Control, Comput., vol. 41, pp. 1426-1435, 2003.

[26] S. Lin and D. Costello, Error Control Coding: Fundamentals and Applications. Prentice-Hall, Englewood Cliffs, NJ, USA, 1983.

[27] B. Xia and W. E. Ryan, "On importance sampling for linear block codes," in Proc. IEEE Int. Conf. Commun. (ICC), vol. 4, pp. 2904-2908 vol.4, May 2003.

[28] B. Xia and W. Ryan, "Importance sampling for tanner trees," IEEE Trans. Inf. Theory, vol. 51, pp. 2183-2189, June 2005.

[29] E. Cavus, C. L. Haymes, and B. Daneshrad, "Low BER performance estimation of LDPC codes via application of importance sampling to trapping sets," IEEE Trans. Commun., vol. 57, pp. 1886-1888, July 2009.

[30] A. Chorti and M. Brookes, "A spectral model for rf oscillators with power-law phase noise," IEEE Transactions on Circuits and Systems I: Regular Papers, vol. 53, no. 9, pp. 1989-1999, 2006.

[31] M. R. Khanzadi, D. Kuylenstierna, A. Panahi, T. Eriksson, and H. Zirath, "Calculation of the performance of communication systems from measured oscillator phase noise," IEEE Transactions on Circuits and Systems I: Regular Papers, vol. 61, no. 5, pp. 1553-1565, 2014.

[32] T. H. Lee and A. Hajimiri, "Oscillator phase noise: a tutorial," IEEE Journal of Solid-State Circuits, vol. 35, no. 3, pp. 326-336, 2000.

[33] X. Chen and A. Wolfgang, "Phase noise mitigation in OFDM-based backhaul in the presence of channel estimation and synchronization errors," in 2016 IEEE 83rd Vehicular Technology Conference (VTC Spring), pp. 1-5, May 2016.

[34] P. Neshaastegaran and A. H. Banihashemi, "Log-likelihood ratio calculation for pilot symbol assisted coded modulation schemes with residual phase noise," IEEE Trans. Commun., vol. 67, pp. 3782-3790, May 2019.

[35] E. L. Lehmann and J. P. Romano, Testing statistical hypotheses. Springer Science \& Business Media, 2006.

[36] B. C. Levy, Principles of signal detection and parameter estimation. Springer Science \& Business Media, 2008. 
[37] S. Landner and O. Milenkovic, "Algorithmic and combinatorial analysis of trapping sets in structured LDPC codes," in Proc. Int. Conf. Wireless Ntwk., Commun., and Mobile Compt., vol. 1, pp. 630-635 vol.1, June 2005.

[38] B. K. Butler and P. H. Siegel, "Error floor approximation for LDPC codes in the AWGN channel," IEEE Trans. Inf. Theory, vol. 60, pp. 7416-7441, Dec 2014.

[39] C. Schlegel and S. Zhang, "On the dynamics of the error floor behavior in (regular) LDPC codes," IEEE Trans. Inf. Theory, vol. 56, pp. 3248-3264, Jul 2010.

[40] Y. Hashemi and A. H. Banihashemi, "New characterization and efficient exhaustive search algorithm for leafless elementary trapping sets of variable-regular LDPC codes," IEEE Trans. Inf. Theory, vol. 62, pp. 6713-6736, Dec 2016.

[41] M. Karimi and A. H. Banihashemi, "Efficient algorithm for finding dominant trapping sets of LDPC codes," IEEE Trans. Inf. Theory, vol. 58, no. 11, pp. 69426958, 2012.

[42] Y. Hashemi and A. Banihashemi, "Characterization of elementary trapping sets in irregular LDPC codes and the corresponding efficient exhaustive search algorithms," IEEE Trans. Inf. Theory, vol. 64, pp. 3411-3430, May 2018.

[43] G. Boiocchi, P. D. Prisco, A. Lahrech, P. Lopez, M. Moretto, and P. Volpato, "Next-generation microwave packet radio: Characteristics and evolution areas to support new scenarios in wireless backhauling," Bell Labs Technical Journal, vol. 18, pp. 143-157, Sept 2013.

[44] N. Kamiya and E. Sasaki, "Pilot-symbol-assisted phase noise compensation with forward-backward wiener smoothing filters," IEEE Transactions on Signal Processing, vol. 65, pp. 4443-4453, Sep. 2017.

[45] V. Simon, A. Senst, M. Speth, and H. Meyr, "Phase noise estimation via adapted interpolation," in Global Telecommunications Conference, 2001. GLOBECOM '01. IEEE, vol. 6, pp. 3297-3301 vol.6, 2001.

[46] J. M. Torrance and L. Hanzo, "Comparative study of pilot symbol assisted modem schemes," in 1995 Sixth International Conference on Radio Receivers and Associated Systems, pp. 36-41, Sep. 1995.

[47] A. Spalvieri and L. Barletta, "Pilot-aided carrier recovery in the presence of phase noise," IEEE Transactions on Communications, vol. 59, pp. 1966-1974, July 2011.

[48] C. N. Georghiades, "Blind carrier phase acquisition for QAM constellations," IEEE Transactions on Communications, vol. 45, no. 11, pp. 1477-1486, 1997.

[49] E. Serpedin, A. Chevreuil, G. B. Giannakis, and P. Loubaton, "Blind channel and carrier frequency offset estimation using periodic modulation precoders," IEEE Trans. Signal Processing, vol. 48, no. 8, pp. 2389-2405, 2000. 
[50] Z. Chen and F. F. Dai, "Effects of LO phase and amplitude imbalances and phase noise on $m$-qam transceiver performance," IEEE Transactions on Industrial Electronics, vol. 57, no. 5, pp. 1505-1517, 2010.

[51] S. Jarboui and S. Hadda, "Blind carrier phase recovery for general quadrature amplitude modulation constellations," IET Communications, vol. 2, pp. 621-629, May 2008.

[52] C. N. Georghiades, "Blind carrier phase acquisition for QAM constellations," IEEE Transactions on Communications, vol. 45, pp. 1477-1486, Nov. 1997.

[53] I. Y. Kushnir, I. M. Bettesh, and Y. Sturkovich, "System, method and apparatus for phase hits and microphonics cancellation," US Patent App. 10/097,188, Oct. 2018.

[54] U. L. Rohde, A. K. Poddar, K. J. Schoepf, R. Rebel, and H. Zhang, "Low noise and low phase hits tunable oscillator," US Patent 7,636,021, Dec. 2009.

[55] D. C. P. S. M. Qin, "System and method for compensation of phase hits," US Patent 11/588,681, May. 2008.

[56] Y. Qin, F. Matsumoto, D. Pham, and C. Easaw, "Phase-hit immunity methods for high speed communication systems," in 2007 IEEE/MTT-S International Microwave Symposium, pp. 1541-1544, June 2007.

[57] K. Andrews, C. Heegard, and D. Kozen, "A theory of interleavers," tech. rep., Cornell University, 1997.

[58] "3GPP TS 38.212 V2.0.0 : Technical specification group radio access network; nr; multiplexing and channel coding (release 15)," 3rd Generation Partnership Project, Dec 2017.

[59] S. Lin and D. J. Costello, Error control coding. Pearson Education India, 2001.

[60] L. Galleani and P. Tavella, "Robust detection of fast and slow frequency jumps of atomic clocks," IEEE Transactions on Ultrasonics, Ferroelectrics, and Frequency Control, vol. 64, pp. 475-485, Feb 2017.

[61] U. Beitler and O. Amrani, "Real time cycle slip detection and correction for APSK modulation," IEEE Transactions on Communications, vol. 62, pp. 736746, February 2014.

[62] D. Mackay, "Encyclopedia of Sparse Graph Codes accessed on Jan. 20, 2020.." Available at: http://www.inference.phy.cam.ac.uk/mackay/codes/data. html.

[63] IEEE Standard for Local and Metropolitan Area Networks Part 16: Air Interface for Fixed Broadband Wireless Access Systems. IEEE Standard 802.16-2004 (Revision of IEEE Std 802.16-2001). 
[64] Digital Video Broadcasting (DVB); Second generation framing structure, channel coding and modulation systems for Broadcasting, Interactive Services, News Gathering and other broadband satellite applications; Part 2: DVB-S2 Extensions (DVB-S2X). ETSI Standard EN 302 307-2 V1.1.1 (2015-02).

[65] T. Richardson and S. Kudekar, "Design of low-density parity check codes for 5G new radio," IEEE Commun. Mag., vol. 56, pp. 28-34, March 2018.

[66] B. J. Frey, R. Kotter, and A. Vardy, "Skewness and pseudocodewords in iterative decoding," in Proc. IEEE Int. Symp. Inf. Theory (ISIT), p. 148, Aug 1998.

[67] T. Richrdson, A. Shokrollahi, and R. Urbanke, "Finite-length analysis of various low-density parity-check ensembles for the binary erasure channel," in Proc. IEEE Int. Symp. Inf. Theory (ISIT), June 2002.

[68] Z. Zhang, L. Dolecek, B. Nikolic, V. Anantharam, and M. Wainwright, "Investigation of error floors of structured low-density parity-check codes via hardware simulation," in Proc. IEEE Glob. Commun. Conf., pp. 1-6, June 2006.

[69] C. Cole, S. G. Wilson, E. Hall, and T. R. Giallorenzi, "A general method for finding low error rates of LDPC codes," 2006. Available at: https://arxiv. org/abs/cs/0605051.

[70] X. Hu, B. V. K. V. Kumar, Z. Li, and R. Barndt, "Error floor estimation of long LDPC codes on partial response channels," in Proc. IEEE Glob. Commun. Conf., pp. 259-264, Nov 2007.

[71] X. Hu, Z. Li, B. V. K. V. Kumar, and R. Barndt, "Error floor estimation of long LDPC codes on magnetic recording channels," IEEE Trans. Magn., vol. 46, pp. 1836-1839, June 2010.

[72] A. Ramamoorthy and N. Varnica, "Error floors of LDPC coded BICM," in Proc. IEEE Int. Conf. Commun. (ICC), pp. 839-844, June 2007.

[73] L. Dolecek, Z. Zhang, M. Wainwright, V. Anantharam, and B. Nikolic, "Evaluation of the low frame error rate performance of LDPC codes using importance sampling," in Proc. IEEE Inf. Theory Wkshp., pp. 202-207, Sep. 2007.

[74] S. Ahn, K. Yang, and D. Har, "Evaluation of the low error-rate performance of LDPC codes over Rayleigh fading channels using importance sampling," IEEE Trans. Commun., vol. 61, pp. 2166-2177, June 2013.

[75] R. Holzlohner, A. Mahadevan, C. R. Menyuk, J. M. Morris, and J. Zweck, "Evaluation of the very low BER of FEC codes using dual adaptive importance sampling," IEEE Commun. Lett., vol. 9, pp. 163-165, Feb 2005.

[76] A. Poloni, S. Valle, and S. Vincenti, "NB-LDPC: Absorbing set and importance sampling," pp. 101-105, 2012. 
[77] A. Alvarado, L. Szczecinski, R. Feick, and L. Ahumada, "Distribution of L-values in gray-mapped $M^{2}-\mathrm{QAM}$ : closed-form approximations and applications," IEEE Trans. Commun., vol. 57, pp. 2071-2079, July 2009.

[78] Yang Han and W. E. Ryan, "LDPC decoder strategies for achieving low error floors," in Proc. IEEE Inf. Theory Appl. Wkshp., pp. 277-286, Jan 2008.

[79] X. Zhang and P. H. Siegel, "Quantized iterative message passing decoders with low error floor for LDPC codes," IEEE Trans. Commun., vol. 62, pp. 1-14, January 2014.

[80] S. K. Chilappagari, S. Sankaranarayanan, and B. Vasic, "Error floors of LDPC codes on the binary symmetric channel," in Proc. IEEE Int. Conf. Commun. (ICC), vol. 3, pp. 1089-1094, June 2006.

[81] T. F. R. M. Tanner, D. Sridhara, "A class of group-structured LDPC codes," in Proc. IEEE Inf. Theory Wkshp., 072001.

[82] Air Interface for Fixed and Mobile Broadband Wireless Access SystemsAmendment for Physical and Medium Access Control Layers for Combined Fixed and Mobile Operation in Licensed Bands. IEEE 802.16 Working Group.

[83] Z. Zhang, L. Dolecek, B. Nikolic, V. Anantharam, and M. J. Wainwright, "Design of LDPC decoders for improved low error rate performance: quantization and algorithm choices," IEEE Trans. Commun., vol. 57, no. 11, pp. 3258-3268, 2009.

[84] G. Boiocchi, P. D. Prisco, A. Lahrech, P. Lopez, M. Moretto, and P. Volpato, "Next-generation microwave packet radio: Characteristics and evolution areas to support new scenarios in wireless backhauling," Bell Labs Technical Journal, vol. 18, pp. 143-157, Sept. 2013. 


\section{Appendix A}

\section{A.1 Derivation of (3.3)}

For the received signal without $\mathrm{PH}$, we have:

$$
r=s e^{j \varphi^{\mathcal{P N}}}+n
$$

Since the pilots are known, we can divide both sides of (A.1) by the deterministic value, $s$. We have:

$$
\frac{r}{s}=e^{j \varphi^{\mathcal{P N}}}+\frac{n}{s}
$$

Equation (A.2) is depicted in Fig. A.1. From (A.2), the following can be written:

$$
\begin{aligned}
\arg \frac{r}{s} & =\arg \left\{e^{j \varphi^{\mathcal{P N}}}+\frac{n}{s}\right\} \\
& =\varphi^{\mathcal{P N}}+\varphi^{\mathcal{W}}
\end{aligned}
$$

Let $\varphi^{\mathcal{P}} \triangleq \arg \frac{r}{s} ;$ thus, $\varphi^{\mathcal{P}}=\varphi^{\mathcal{P N}}+\varphi^{\mathcal{W}}$.

If we decompose the vector $n$ into tangential $\left(n_{t}\right)$ and radial $\left(n_{r}\right)$ components, for the total phase deflection at the pilot location $\left(\varphi^{\mathcal{P}}\right)$, the following formula can be written:

$$
e^{j \varphi^{\mathcal{P}}}=e^{j \varphi^{\mathcal{P N}}}+\frac{n_{t}}{s}
$$

This is also shown in Fig. A.2. Note that on the unit circle, the angle (in radians) is equal to the length of the arch, which is covered by that angle. Here the arch which is covered by the angle $\varphi^{\mathcal{W}}$ can be approximated by $\frac{n_{t}}{s}$. We also approximate $n_{t}$ to 


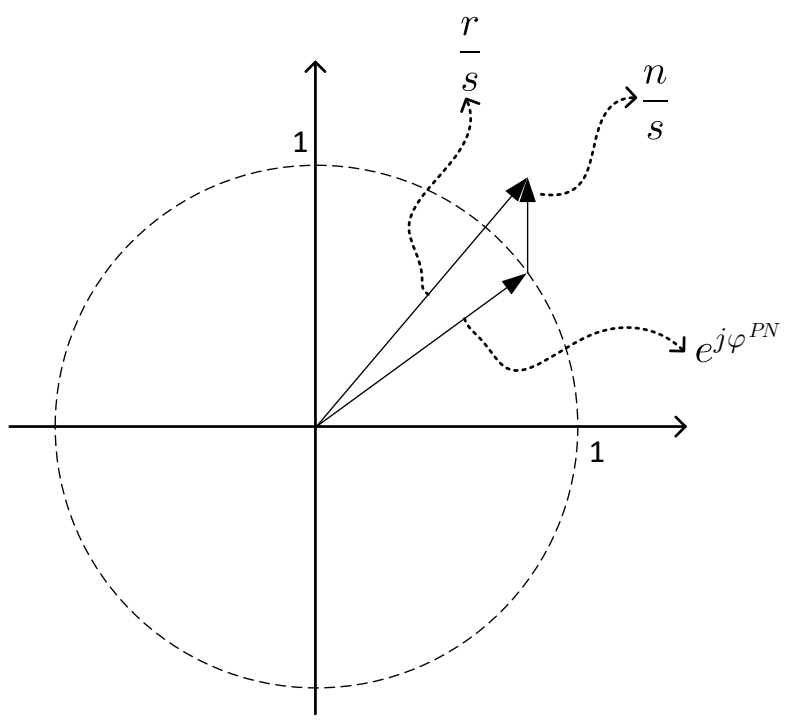

Figure A.1: The phase offset on the received signals are decomposed into Gaussian noise component and $\mathrm{PN}$ component.

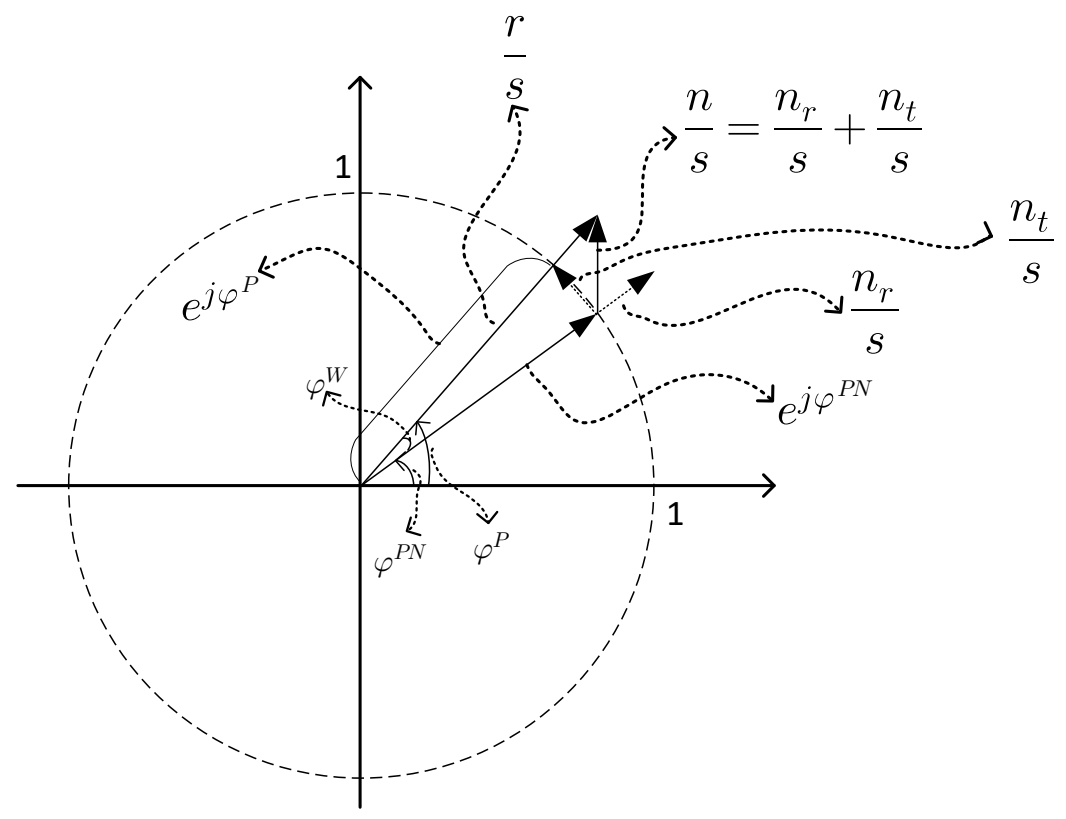

Figure A.2: Various elements involved in (A.3) are decomposed into radial and tangential components. 
be a Gaussian random variable with mean 0 and variance equal to half the variance of $n$. By comparing (A.3) and (A.4) we have:

$$
\varphi^{\mathcal{W}} \approx \frac{n_{t}}{s},
$$

where $n_{t} \sim \mathcal{N}\left(0, \frac{N_{0}}{2}\right)$. To find the first and the second moments of $\varphi^{\mathcal{W}}$, we need to condition on $s$ and use the total probability theorem.

$$
\begin{aligned}
\mathcal{E}\left\{\varphi^{\mathcal{W}}\right\}=\mathcal{E}\left\{\frac{n_{t}}{s}\right\} & =\sum_{j=1}^{\mathcal{M}} \mathcal{E}\left\{\frac{n_{t}}{s} \mid s=s_{j}\right\} \cdot \mathcal{P}\left(s=s_{j}\right) \\
& =\frac{1}{\mathcal{M}} \sum_{j=1}^{\mathcal{M}} \frac{\mathcal{E}\left\{n_{t}\right\}}{s_{j}} \\
& =0
\end{aligned}
$$

For the second moment, we have:

$$
\begin{aligned}
\mathcal{E}\left\{\left|\frac{n_{t}}{s}\right|^{2}\right\} & =\sum_{j=1}^{\mathcal{M}} \mathcal{E}\left\{\left|\frac{n_{t}}{s}\right|^{2} \mid s=s_{j}\right\} \cdot \mathcal{P}\left(s=s_{j}\right) \\
& =\frac{1}{\mathcal{M}} \sum_{j=1}^{\mathcal{M}} \frac{\mathcal{E}\left\{\left|n_{t}\right|^{2}\right\}}{\left|s_{j}\right|^{2}} \\
& =\mathcal{E}\left\{\left|n_{t}\right|^{2}\right\} \times\left[\frac{1}{\mathcal{M}} \sum_{j=1}^{\mathcal{M}} \frac{1}{\left|s_{j}\right|^{2}}\right] \\
& =\mathcal{E}\left\{\left|n_{t}\right|^{2}\right\} \times \mathcal{E}\left\{|s|^{-2}\right\} \\
& \approx \mathcal{E}\left\{|n|^{2} / 2\right\} \times \mathcal{E}\left\{|s|^{-2}\right\} .
\end{aligned}
$$

From (A.6) and (A.7) we calculate the variance of $\varphi^{\mathcal{W}}$ as follows:

$$
\operatorname{var}\left\{\varphi^{\mathcal{W}}\right\}=\mathcal{E}\left\{|n|^{2} / 2\right\} \cdot \mathcal{E}\left\{|s|^{-2}\right\},
$$

Note that for the pilot locations, we choose the transmitted signals from a smaller constellation. The symbols corresponding to this constellation are denoted by $s_{p}$ (rather than $s$ ). Hence, by using (A.5), (A.6), and (A.8) we have $\varphi^{\mathcal{W}} \sim \mathcal{N}\left(0, \mathcal{E}\left\{|n|^{2} / 2\right\}\right.$. $\left.\mathcal{E}\left\{\left|s_{p}\right|^{-2}\right\}\right)$. 


\section{A.2 Derivation of (3.18)}

We begin with defining the functions $g(\cdot)$ and $h(\cdot)$ as follows:

$$
g(a) \triangleq \int_{x=0}^{a} \mathrm{Q}(x) d x, \quad h(a, b, c) \triangleq \int_{x=0}^{a} \mathrm{Q}\left(\frac{x+b}{c}\right) d x
$$

After some manipulations using basic calculus we have:

$$
h(a, b, c)=c\left[g\left(\frac{a+b}{c}\right)-g\left(\frac{b}{c}\right)\right]
$$

The probability of DM is calculated by combining (2.13) and (3.13) as follows:

$$
\begin{aligned}
\min \operatorname{Pr}(\mathrm{DM}) & =\frac{1}{d_{M}-d_{m}} \int_{0}^{\gamma_{N P}}\left[\mathrm{Q}\left(\frac{d_{m}-z}{\sigma_{P}}\right)\right. \\
& \left.+\mathrm{Q}\left(\frac{d_{m}+z}{\sigma_{P}}\right)-\mathrm{Q}\left(\frac{d_{M}-z}{\sigma_{P}}\right)-\mathrm{Q}\left(\frac{d_{M}+z}{\sigma_{P}}\right)\right] d z
\end{aligned}
$$

where $\gamma_{N P}$ is calculated from (3.15). Since $\mathrm{Q}(-x)=1-\mathrm{Q}(x)$, the integral in (A.11) can be written as follows:

$$
\begin{aligned}
\min \operatorname{Pr}(\mathrm{DM}) & =\frac{1}{d_{M}-d_{m}} \int_{0}^{\gamma_{N P}}\left[\gamma_{N P}-\mathrm{Q}\left(\frac{z-d_{m}}{\sigma_{P}}\right)\right. \\
& +\mathrm{Q}\left(\frac{d_{m}+z}{\sigma_{P}}\right)-\gamma_{N P} \\
& \left.+\mathrm{Q}\left(\frac{z-d_{M}}{\sigma_{P}}\right)-\mathrm{Q}\left(\frac{d_{M}+z}{\sigma_{P}}\right)\right] d z
\end{aligned}
$$

Conditioning on calculation of $g(a)$, by letting $a=\gamma_{N P}, b=d_{m}$ (or $d_{M}$ ) and $c=\sigma_{P}$ and combining (A.9) and (A.12) we have:

$$
\begin{aligned}
\min \operatorname{Pr}(\mathrm{DM}) & =\frac{1}{d_{M}-d_{m}}\left[h\left(\gamma_{N P}, d_{m}, \sigma_{P}\right)+h\left(\gamma_{N P},-d_{M}, \sigma_{P}\right)\right. \\
& \left.-h\left(\gamma_{N P},-d_{m}, \sigma_{P}\right)-h\left(\gamma_{N P}, d_{M}, \sigma_{P}\right)\right]
\end{aligned}
$$


By combining (A.13) and (A.10) we have:

$$
\begin{aligned}
\min \operatorname{Pr}(\mathrm{DM}) & =\frac{\sigma_{P}}{d_{M}-d_{m}}\left[g\left(\frac{\gamma_{N P}+d_{m}}{\sigma_{P}}\right)-g\left(\frac{d_{m}}{\sigma_{P}}\right)\right. \\
& +g\left(\frac{\gamma_{N P}-d_{M}}{\sigma_{P}}\right)-g\left(-\frac{d_{M}}{\sigma_{P}}\right)+g\left(-\frac{d_{m}}{\sigma_{P}}\right) \\
& \left.-g\left(\frac{\gamma_{N P}-d_{m}}{\sigma_{P}}\right)+g\left(\frac{d_{M}}{\sigma_{P}}\right)-g\left(\frac{\gamma_{N P}+d_{M}}{\sigma_{P}}\right)\right] .
\end{aligned}
$$

To calculate the function $g(a)$, the first integral in (A.9) is expanded as follows:

$$
\begin{aligned}
g(a) & =\int_{x=0}^{a} \int_{y=x}^{\infty} \frac{1}{\sqrt{2 \pi}} e^{-\frac{y^{2}}{2}} d y d x \\
& =\int_{y=0}^{a} \frac{1}{\sqrt{2 \pi}} e^{-\frac{y^{2}}{2}} d y \int_{x=0}^{y} d x+\int_{y=a}^{\infty} \frac{1}{\sqrt{2 \pi}} e^{-\frac{y^{2}}{2}} d y \int_{x=0}^{a} d x \\
& =\int_{y=0}^{a} \frac{1}{\sqrt{2 \pi}} y e^{-\frac{y^{2}}{2}} d y+a \int_{y=a}^{\infty} \frac{1}{\sqrt{2 \pi}} e^{-\frac{y^{2}}{2}} d y \\
& =\frac{1}{\sqrt{2 \pi}}\left[1-e^{-\frac{a^{2}}{2}}\right]+a \mathrm{Q}(a) .
\end{aligned}
$$

To derive (3.18), we replace the function $g(\cdot)$ from (A.15) in (A.14). Subsequently, by defining a new auxiliary function $\hat{g}(\cdot)$, where $\hat{g}(a) \triangleq a \mathrm{Q}(a)-\frac{1}{\sqrt{2 \pi}} e^{\frac{-a^{2}}{2}}$, and after some manipulations the analytic expression of probability of DM after using the NPBHT is obtained. 


\section{Appendix B}

\section{B.1 Derivation of (4.30)}

To calculate $\operatorname{Pr}(\mathrm{T}$-weak codeword), we begin by investigating the relative level of protection that the VNs of a TS receive from the constellation. In particular, the protection of an arbitrary constellation point at a given BL is considered. This protection is a function of Euclidean distance of the constellation points and the bitto-symbol mapping scheme. In Table B.1 the bit-to-symbol mapping for the Gray mapped 16-PAM constellation is displayed. The bold binary numbers indicate the BLs, where each symbol is weak at them (cf. Definition 3). By careful inspection of this table, we determine the number of weak symbols at each BL. The number for the MSB position is always 2 while for the LSB position is equal to the size of the signal set, i.e., $M$. The Gray labeling dictates that for the $b$-th BL, the number of weak symbols is $2^{b}$ where $1 \leq b \leq m$ and $m=\log _{2} M$. Same argument applies to any Gray labeled PAM constellations with arbitrary size. Hence, for an $M-\mathrm{PAM}$ constellation, the probability of a symbol being weak at $b$-th position is $2^{b-m}$. With respect to the codewords, we treat the codeword generation as the process of picking one sample (codeword) from a pool of i.i.d. samples (all the possible codewords). This sample is either a T-weak codeword or it is not. For the T-weak codewords all the symbols corresponding to $\mathrm{T}$ are weak at their respective BLs. Hence, we obtain:

$$
\begin{aligned}
\operatorname{Pr}(\mathrm{T} \text {-weak codeword }) & =\operatorname{Pr}\left(\bigcap_{i=1}^{a} s_{\mathrm{T}}^{i} \text { is a weak symbol at BL } \pi_{\mathrm{T}}^{i}\right) \\
& =\prod_{i=1}^{a} 2^{\left(\pi_{\mathrm{T}}^{i}-m\right)} .
\end{aligned}
$$




\begin{tabular}{|c|c|c|c|c|c|c|c|c|c|c|c|c|c|c|c|c|}
\hline coordinate of the symbol & -15 & -13 & -11 & -9 & -7 & -5 & -3 & -1 & +1 & +3 & +5 & +7 & +9 & +11 & +13 & +15 \\
\hline BL 1 (MSB) & 0 & 0 & 0 & 0 & 0 & 0 & 0 & $\mathbf{0}$ & $\mathbf{1}$ & 1 & 1 & 1 & 1 & 1 & 1 & 1 \\
\hline BL 2 & 0 & 0 & 0 & $\mathbf{0}$ & $\mathbf{1}$ & 1 & 1 & 1 & 1 & 1 & 1 & $\mathbf{1}$ & $\mathbf{0}$ & 0 & 0 & 0 \\
\hline BL 3 & 0 & $\mathbf{0}$ & $\mathbf{1}$ & 1 & 1 & $\mathbf{1}$ & $\mathbf{0}$ & 0 & 0 & $\mathbf{0}$ & $\mathbf{1}$ & 1 & 1 & $\mathbf{1}$ & $\mathbf{0}$ & 0 \\
\hline BL 4 (LSB) & $\mathbf{1}$ & $\mathbf{0}$ & $\mathbf{0}$ & $\mathbf{1}$ & $\mathbf{1}$ & $\mathbf{0}$ & $\mathbf{0}$ & $\mathbf{1}$ & $\mathbf{1}$ & $\mathbf{0}$ & $\mathbf{0}$ & $\mathbf{1}$ & $\mathbf{1}$ & $\mathbf{0}$ & $\mathbf{0}$ & $\mathbf{1}$ \\
\hline
\end{tabular}

Table B.1: 16-PAM constellation and the bits mapped to each symbol

\section{B.2 Tables Corresponding to Figs. $4.4-4.6$}

The following Tables correspond to Figs. 4.4 - 4.6. These tables provide similar information for Figs. 4.4 - 4.6 to what is provided for Tanner code in Table 4.2. In particular, in each row, the left column includes the TS class, the multiplicity of TSs in that class followed by the number of non-isomorphic structures in parentheses, and a coefficient (e.g., $10^{6}$ ), which should be multiplied by the corresponding numbers in the same row. The table dedicates a column to every constellation. The first row of each dedicated column indicates the constellation size, and the SNRs, e.g., for 8-PAM signalling, three SNRs are considered, namely, 9, 10, and $11 \mathrm{~dB}$. The last row of each dedicated column indicates the simulation gain achieved by using the proposed EF estimation scheme, when compared to conventional MC method. The numbers are calculated using (4.10). The rest of rows except the first and the last one, contain the information corresponding to each TS class. In particular, the numerical values of three parameters are provided. In the first row, the number of extracted PDTSs is provided. In the second row, the relative error rate contribution of TS class at each SNR is provided. In the third row, the total number of trials to estimate the error rate of TS class is provided. For example, when using 8-PAM signalling, 5 TSs out of 6 non-isomorphic ETSs in class $(4,2)$ are chosen by the PDTS extraction technique. At a SNR of $9 \mathrm{~dB}$, the class $(4,2)$ contributes to $19 \%$ of the error rate. In this case, the error rate is estimated by performing a total of $2.4 \times 10^{6}$ trials. 


\begin{tabular}{|c|c|c|c|c|c|}
\hline $\begin{array}{c}\text { Constellation size } \\
\text { SNR values }\end{array}$ & $\begin{array}{c}8-\mathrm{PAM} \\
91011\end{array}$ & $\begin{array}{c}16-\mathrm{PAM} \\
141516\end{array}$ & $\begin{array}{c}32-\mathrm{PAM} \\
171819\end{array}$ & $\begin{array}{c}64-\mathrm{PAM} \\
20.521 .522 .5\end{array}$ & $\begin{array}{c}128-\mathrm{PAM} \\
2424.525\end{array}$ \\
\hline$(4,2)$ & 5 & 2 & 3 & 3 & 2 \\
$6(1)$ & $19 \% 44 \% 64.5 \%$ & $74 \% 82 \% 91 \%$ & $3 \% 11 \% 18 \%$ & $73 \% 87 \% 95 \%$ & $10 \% 9 \% 7 \%$ \\
$10^{6} \times$ & 2.42 .42 .15 & 2.11 .851 .8 & 2.182 .131 .95 & 3.643 .12 .87 & 3.53 .52 .85 \\
\hline$(6,2)$ & 5 & 3 & 1 & 1 & 1 \\
$5(1)$ & $74 \% 53 \% 35.3 \%$ & $26 \% 18 \% 9 \%$ & $84 \% 82 \% 79 \%$ & $27 \% 13 \% 5 \%$ & $90 \% 91 \% 93 \%$ \\
$10^{6} \times$ & 8.728 .578 .97 & 6.897 .367 .76 & 0.20 .30 .5 & 2.661 .821 .88 & 0.650 .651 \\
\hline$(8,2)$ & 2 & & 1 & & \\
$3(1)$ & $7 \% 3 \% 0.2 \%$ & - & $13 \% 7 \% 3 \%$ & - & - \\
$10^{6} \times$ & 8.28 .28 .21 & & 8.078 .078 & & \\
\hline Simulation gain $(\mathrm{dB})$ & -4.215 .7 & 3.6810 .7 & 049.5 & 9.211 .512 .2 & 11.112 .414 .6 \\
\hline
\end{tabular}

Table B.2: Error floor estimation of the MacKay $(1008,504)$ LDPC code using $M-\mathrm{PAM}$ signalling. The number of estimated TSs, the relative contribution of a given TS in the total estimated FER, and the total number of trials for the estimation of a TS class are provided in each case. 


\begin{tabular}{|c|c|c|c|c|c|}
\hline $\begin{array}{l}\text { Constellation size } \\
\text { SNR values }\end{array}$ & $\begin{array}{c}\text { 8-PAM } \\
10.51111 .5\end{array}$ & $\begin{array}{c}16-\mathrm{PAM} \\
14.51515 .5\end{array}$ & $\begin{array}{l}32-\mathrm{PAM} \\
212223 \\
\end{array}$ & $\begin{array}{l}64-\mathrm{PAM} \\
2424.525\end{array}$ & $\begin{array}{l}128-\mathrm{PAM} \\
2929.530 \\
\end{array}$ \\
\hline$(8,1)$ & 1 & 1 & & 1 & 1 \\
\hline 84 & $43.8 \% 35.8 \% 17 \%$ & $49 \% 66.8 \% 68.3 \%$ & - & $79.5 \% 78.9 \% 77.8 \%$ & $79.2 \% 84.7 \% 91.1 \%$ \\
\hline $10^{5} \times$ & 0.050 .10 .2 & 0.050 .050 .05 & & 0.10 .10 .1 & 0.050 .050 .05 \\
\hline$(9,1)$ & 2 & 2 & & 2 & 2 \\
\hline 168 & $10.2 \% 5.8 \% 2.3 \%$ & $5 \% 4.2 \% 3.4 \%$ & - & $12.8 \% 9.8 \% 6 \%$ & $12.2 \% 9.1 \% 5 \%$ \\
\hline $10^{5} \times$ & 1.11 .11 .1 & 0.20 .250 .35 & & 0.20 .20 .2 & 0.250 .350 .85 \\
\hline$(3,2)$ & 4 & 4 & 5 & 5 & 4 \\
\hline 504 & $1.8 \% 8.4 \% 23.4 \%$ & $0.5 \% 1.1 \% 2.6 \%$ & $68.1 \% 77.3 \% 86.6 \%$ & $0 \% \quad 0 \% \quad 0.1 \%$ & $0 \% 0.8 \% 1.1 \%$ \\
\hline $10^{5} \times$ & $0.25 \quad 0.20 .2$ & 0.60 .80 .8 & 111.25 & 1.61 .21 .4 & 1.61 .51 .6 \\
\hline$(4,2)$ & 6 & 6 & 8 & 4 & 4 \\
\hline 588 & $4.2 \% 13.7 \% 27.4 \%$ & $0.1 \% \quad 0.6 \% 0.6 \%$ & $22.8 \% \quad 18.7 \% 12 \%$ & $0 \% 0.1 \% 0.4 \%$ & $0 \% 0 \% 0.1 \%$ \\
\hline $10^{5} \times$ & 0.40 .30 .3 & 2.22 .23 .2 & 22.22 .35 & 1.82 .42 .4 & 1.40 .650 .4 \\
\hline$(5,2)$ & 5 & 5 & 12 & 5 & 4 \\
\hline 756 & $4 \% 9.7 \% 13.2 \%$ & $0.5 \% 1.1 \% 1.3 \%$ & $7.7 \% \quad 3.5 \% \quad 1.3 \%$ & $0 \% 0.1 \% 0.5 \%$ & $2.8 \% 1 \% \quad 0.2 \%$ \\
\hline $10^{5} \times$ & 0.250 .250 .25 & 1.41 .51 .4 & 7.67 .17 .2 & 332.7 & 1.10 .30 .25 \\
\hline$(6,2)$ & 9 & 9 & 20 & 7 & 4 \\
\hline 1008 & $1.9 \% 4.2 \% 4.4 \%$ & $0.8 \% \quad 0.6 \% \quad 0.2 \%$ & $1.4 \% \quad 0.5 \% \quad 0.1 \%$ & $1.3 \% 1 \% 0.9 \%$ & $0.4 \% \quad 0.3 \% \quad 0.3 \%$ \\
\hline $10^{5} \times$ & 0.450 .550 .55 & 443.8 & 121212 & 4.24 .13 .8 & 0.90 .90 .9 \\
\hline$(7,2)$ & & 9 & & 4 & \\
\hline 1344 & - & $3.7 \% \quad 1.2 \% \quad 0.9 \%$ & - & $2 \% \quad 0.7 \% 1 \%$ & - \\
\hline $10^{5} \times$ & & 3.73 .73 .55 & & 2.32 .11 .8 & \\
\hline$(8,2)$ & 27 & 36 & & 23 & 7 \\
\hline 1932 & $23 \% 14.7 \% 10.2 \%$ & $29.4 \% \quad 16.2 \% 14.6 \%$ & - & $3 \% 8.1 \% 12.7 \%$ & $5.4 \% 4.1 \% 2.2 \%$ \\
\hline $10^{5} \times$ & 7.29 .912 .1 & 15.515 .415 .6 & & 13.413 .213 & 5.65 .15 .6 \\
\hline$(9,2)$ & 47 & 44 & & 36 & \\
\hline 2772 & $11.1 \% 7.7 \% 2.1 \%$ & $11 \% 8.2 \% 8.1 \%$ & - & $1.4 \% 1.3 \% 0.6 \%$ & - \\
\hline $10^{6} \times$ & 2.372 .512 .58 & 18.618 .518 .5 & & 2.722 .722 .71 & \\
\hline Simulation gain $(\mathrm{dB})$ & 7.711 .315 .5 & -1.82 .67 .8 & 6.59 .315 .6 & 46.511 .2 & 10.514 .117 .6 \\
\hline
\end{tabular}

Table B.3: Error floor estimation of the WiMAX1 $(2016,1344)$ LDPC code using $M-\mathrm{PAM}$ signalling. The number of estimated TSs, the relative contribution of a given TS in the total estimated FER, and the total number of trials for the estimation of a TS class are provided in each case. 


\begin{tabular}{|c|c|c|c|c|c|}
\hline $\begin{array}{c}\text { Constellation size } \\
\text { SNR values }\end{array}$ & $8-$ PAM & 16 -PAM & $32-$ PAM & 64 -PAM & 128 -PAM \\
\hline$(3,1)$ & 23.514 & 1818.519 & 2222.523 & 2727.528 & 3232.533 \\
48 & $86.3 \% 92.7 \% 95.8 \%$ & $77.7 \% 79.2 \% 86 \%$ & $89.1 \% 93 \% 97.3 \%$ & $94.5 \% 97 \% 97.2 \%$ & $82.6 \% 85.2 \% 91.5 \%$ \\
$10^{5} \times$ & 0.20 .30 .65 & 0.951 .21 .4 & 11.72 .1 & 0.10 .150 .15 & 0.81 .11 .45 \\
\hline$(4,1)$ & 8 & 3 & 15 & 7 & 8 \\
144 & $12.5 \% 6.5 \% 3.4 \%$ & $17.5 \% 16 \% 11 \%$ & $8 \% 5.3 \% 1.6 \%$ & $5.1 \% 3 \% 2.8 \%$ & $15.3 \% 13.7 \% 7.6 \%$ \\
$10^{5} \times$ & 3.654 .354 .4 & 1.21 .51 .65 & 7.257 .557 .7 & 3.43 .453 .55 & 1.9522 .4 \\
\hline$(5,1)$ & 10 & 2 & 7 & 5 & 4 \\
144 & $0.2 \% 0.1 \% 0 \%$ & $2.3 \% 2.9 \% 1.7 \%$ & $0.1 \% 0 \% 0 \%$ & $0.4 \% 0 \% 0 \%$ & $1.5 \% 0.8 \% 0.5 \%$ \\
$10^{5} \times$ & 5.55 .55 .5 & 1.11 .11 .1 & 3.853 .653 .3 & 2.752 .752 .75 & 2.152 .22 .2 \\
\hline$(3,2)$ & 10 & 8 & & & 8 \\
240 & $0.3 \% 0.2 \% 0.5 \%$ & $0.6 \% 0.8 \% 1 \%$ & $0.2 \% 0.3 \% 0.3 \%$ & - & $0.1 \% 0.2 \% 0.3 \%$ \\
$10^{5} \times$ & 0.70 .81 .4 & 0.60 .61 .15 & 11.11 .45 & & 0.450 .450 .45 \\
\hline$(4,2)$ & 26 & 22 & & & 12 \\
\hline 960 & $0.7 \% 0.5 \% 0.3 \%$ & $1.9 \% 1.1 \% 0.3 \%$ & $2.6 \% 1.4 \% 0.8 \%$ & - & $0.5 \% 0.3 \% 0.1 \%$ \\
$10^{5} \times$ & 89.912 .5 & 7.89 .8510 .6 & 12.614 .217 .7 & & 1.61 .751 .95 \\
\hline Simulation gain (dB) & 8.512 .115 .7 & 1720.223 .9 & 9.11316 .7 & 12.31618 & 20.121 .823 .3 \\
\hline
\end{tabular}

Table B.4: Error floor estimation of the WiMAX2 $(1152,960)$ LDPC code using $M-\mathrm{PAM}$ signalling. The number of estimated TSs, the relative contribution of a given TS in the total estimated FER, and the total number of trials for the estimation of a TS class are provided in each case. 


\section{Appendix $\mathrm{C}$}

\section{C.1 Tables Corresponding to Figs. 5.1 - 5.4}

The following Tables correspond to Figs. 5.1 - 5.4. These tables provide similar information for Figs. 5.1 - 5.4 to what is provided for PAM-based systems in Appendix B.2.

\begin{tabular}{|c|c|c|c|c|}
\hline $\begin{array}{c}\text { Constellation size } \\
\text { SNR values }\end{array}$ & $\begin{array}{c}16-\mathrm{QAM} \\
8910\end{array}$ & $\begin{array}{c}64-\mathrm{QAM} \\
111213\end{array}$ & $\begin{array}{c}256-\mathrm{QAM} \\
151617\end{array}$ & $\begin{array}{c}1024-\mathrm{QAM} \\
18.51919 .5\end{array}$ \\
\hline$(8,2)$ & 89 & 75 & 28 & 24 \\
$465(1)$ & $87.8 \% 94 \% 98 \%$ & $79.6 \% 84.8 \% 85 \%$ & $90.8 \% 93.1 \% 99 \%$ & $68.5 \% 74.5 \% 77.7 \%$ \\
$10^{6} \times$ & 4.744 .844 .85 & 3.563 .73 .55 & 1.521 .61 .52 & 1.471 .521 .57 \\
\hline$(10,2)$ & 279 & 168 & 34 & 28 \\
$1395(2)$ & $12.2 \% 5.9 \% 1.9 \%$ & $20.3 \% 15.1 \% 15 \%$ & $9.2 \% 6.9 \% 1 \%$ & $31.5 \% 25.5 \% 22.6 \%$ \\
$10^{6} \times$ & 15.3414 .9313 .61 & 9.68 .98 .3 & 2.672 .322 & 4.44 .234 .05 \\
\hline Simulation gain $(\mathrm{dB})$ & -64.618 & -8.1110 .3 & 1.69 .519 & 1.33 .57 .9 \\
\hline
\end{tabular}

Table C.1: Error floor estimation of the Tanner $(155,62)$ LDPC code using $M-\mathrm{QAM}$ signalling. The number of estimated TSs, the relative contribution of a given TS in the total estimated FER, and the total number of trials for the estimation of a TS class are provided in each case. 


\begin{tabular}{|c|c|c|c|c|}
\hline $\begin{array}{c}\text { Constellation size } \\
\text { SNR values }\end{array}$ & $\begin{array}{c}16-\mathrm{QAM} \\
77.58\end{array}$ & $\begin{array}{c}64-\mathrm{QAM} \\
1010.511\end{array}$ & $\begin{array}{c}256-\mathrm{QAM} \\
14.51515 .5\end{array}$ & $\begin{array}{c}1024-\mathrm{QAM} \\
1717.518\end{array}$ \\
\hline$(4,2)$ & 5 & 5 & 4 & 5 \\
$6(1)$ & $77.3 \% 83.2 \% 88.8 \%$ & $39.5 \% 64.3 \% 67 \%$ & $85.6 \% 94.3 \% 93.5 \%$ & $5.8 \% 7.4 \% 7.7 \%$ \\
$10^{6} \times$ & 0.250 .30 .25 & 0.751 .050 .65 & 21.951 .95 & 2.752 .752 .75 \\
\hline$(6,2)$ & 5 & 5 & 5 & 1 \\
$5(1)$ & $22.7 \% 16.8 \% 11.2 \%$ & $60.5 \% 35.8 \% 33 \%$ & $14.4 \% 5.7 \% 6.5 \%$ & $79.2 \% 83.7 \% 84.7 \%$ \\
$10^{6} \times$ & 0.450 .650 .75 & 5.24 .26 & 2.852 .852 .85 & 0.550 .550 .55 \\
\hline$(8,2)$ & & - & & 1 \\
$3(1)$ & - & - & - & $15 \% 8.9 \% 7.6 \%$ \\
$10^{6} \times$ & & & & 0.550 .550 .55 \\
\hline Simulation gain $(\mathrm{dB})$ & 16.417 .520 .3 & 5.1810 .6 & 810.812 .5 & 4.86 .19 \\
\hline
\end{tabular}

Table C.2: Error floor estimation of the MacKay $(1008,504)$ LDPC code using $M-\mathrm{QAM}$ signalling. The number of estimated TSs, the relative contribution of a given TS in the total estimated FER, and the total number of trials for the estimation of a TS class are provided in each case. 


\begin{tabular}{|c|c|c|c|c|}
\hline $\begin{array}{l}\text { Constellation size } \\
\text { SNR values }\end{array}$ & $\begin{array}{c}\text { 16-QAM } \\
88.59\end{array}$ & $\begin{array}{l}64-\mathrm{QAM} \\
1010.511\end{array}$ & $\begin{array}{c}256-\mathrm{QAM} \\
14.51515 .5\end{array}$ & $\begin{array}{c}\text { 1024-QAM } \\
212223\end{array}$ \\
\hline$(8,1)$ & 2 & 1 & 1 & \\
\hline 84 & $5.3 \% 1.1 \% 0.4 \%$ & $35.8 \% 46.5 \% 42.2 \%$ & $45.5 \% 65.3 \% 50 \%$ & - \\
\hline $10^{5} \times$ & 0.80 .80 .8 & 0.20 .20 .2 & 0.10 .10 .1 & \\
\hline$(9,1)$ & 5 & 2 & 2 & \\
\hline 168 & $0.04 \% 0.01 \% 0.001 \%$ & $2.9 \% 2.3 \% 1.3 \%$ & $3.9 \% 4 \% 1.8 \%$ & - \\
\hline $10^{5} \times$ & 3.353 .32 .7 & 1.44 .44 .4 & 0.20 .20 .4 & \\
\hline$(3,2)$ & 3 & 4 & 4 & 5 \\
\hline 504 & $48 \% 63 \% 71.5 \%$ & $0.08 \% \quad 0.5 \% 1.5 \%$ & $0.05 \% 0.18 \% 0.4 \%$ & $69 \% 80 \% 88.9 \%$ \\
\hline $10^{5} \times$ & 0.150 .150 .15 & 0.80 .80 .8 & 1.150 .50 .3 & 3.63 .13 .5 \\
\hline$(4,2)$ & 5 & 6 & 6 & 8 \\
\hline 588 & $28.7 \% 25.7 \% 22.5 \%$ & $1.6 \% 1.4 \% 2.8 \%$ & $0.18 \% 0.65 \% 1.1 \%$ & $22.7 \% 16.8 \% 10 \%$ \\
\hline $10^{5} \times$ & $\begin{array}{llll}0.25 & 0.25 & 0.25\end{array}$ & 3.21 .22 .2 & 0.650 .450 .3 & 5.45 .25 .4 \\
\hline$(5,2)$ & 7 & 5 & 5 & 12 \\
\hline 756 & $10.3 \% 7.2 \% 4.5 \%$ & $0.6 \% 1.2 \% 1.9 \%$ & $0.2 \% 0.37 \% 0.7 \%$ & $6.8 \% 2.9 \% 1 \%$ \\
\hline $10^{5} \times$ & 0.350 .350 .35 & 2.411 & 0.250 .30 .25 & 8.18 .89 .6 \\
\hline$(6,2)$ & 18 & 9 & 5 & 20 \\
\hline 1008 & $2.1 \% 1.1 \% 0.6 \%$ & $2.7 \% \quad 0.7 \% \quad 0.7 \%$ & $0.7 \% 0.3 \% 0.25 \%$ & $1.4 \% 0.45 \% \quad 0.1 \%$ \\
\hline $10^{5} \times$ & 0.911 .05 & 3.82 .82 .8 & 0.750 .250 .3 & 17.317 .819 .6 \\
\hline$(7,2)$ & 24 & 12 & 6 & \\
\hline 1344 & $2.2 \% \quad 0.7 \% \quad 0.2 \%$ & $5.6 \% 2.3 \% 0.15 \%$ & $3.3 \% 0.1 \% 0.05 \%$ & - \\
\hline $10^{5} \times$ & 2.852 .353 .05 & 4.44 .42 .4 & 10.450 .5 & \\
\hline$(8,2)$ & 34 & 27 & 36 & \\
\hline 1932 & $2.3 \% \quad 0.7 \% 0.2 \%$ & $24 \% 17 \% 29 \%$ & $22.9 \% 13.6 \% 41.3 \%$ & - \\
\hline $10^{5} \times$ & 5.67 .17 .75 & 11.812 .619 .6 & 38.237 .135 .8 & \\
\hline$(9,2)$ & 66 & 47 & 44 & \\
\hline 2772 & $1 \% 0.3 \% 0.03 \%$ & $26.6 \% 28 \% 20 \%$ & $23.9 \% 15.5 \% 4.4 \%$ & - \\
\hline $10^{6} \times$ & 2.172 .212 .77 & 6.187 .648 .96 & 4.84 .74 .67 & \\
\hline Simulation gain $(\mathrm{dB})$ & 18.621 .421 .7 & 0.15 .19 .8 & 2.97 .312 .1 & 4.8812 .2 \\
\hline
\end{tabular}

Table C.3: Error floor estimation of the WiMAX1 $(2016,1344)$ LDPC code using $M-\mathrm{QAM}$ signalling. The number of estimated TSs, the relative contribution of a given TS in the total estimated FER, and the total number of trials for the estimation of a TS class are provided in each case. 


\begin{tabular}{|c|c|c|c|c|}
\hline $\begin{array}{l}\text { Constellation size } \\
\text { SNR values }\end{array}$ & $\begin{array}{c}\text { 16-QAM } \\
8.599 .5\end{array}$ & $\begin{array}{l}64-\mathrm{QAM} \\
1313.514\end{array}$ & $\begin{array}{c}256-\mathrm{QAM} \\
1717.518\end{array}$ & $\begin{array}{l}\text { 1024-QAM } \\
21.52222 .5\end{array}$ \\
\hline$(3,1)$ & 2 & 2 & 5 & 6 \\
\hline 48 & $31.9 \% 58.4 \% 75.5 \%$ & $82.2 \% 87 \% 92.3 \%$ & $34 \% 48 \% 60.1 \%$ & $16.4 \% 18.4 \% 64.3 \%$ \\
\hline $10^{5} \times$ & 0.10 .10 .1 & 0.10 .10 .1 & 0.460 .520 .39 & 0.650 .751 \\
\hline$(4,1)$ & 2 & 9 & 16 & 9 \\
\hline 144 & $17.6 \% 17 \% 13.6 \%$ & $11.8 \% 9.2 \% 6.6 \%$ & $36.4 \% 34.7 \% 31.8 \%$ & $2.9 \% 3.3 \% 5.4 \%$ \\
\hline $10^{5} \times$ & 0.20 .10 .1 & 0.450 .450 .45 & 1.51 .551 .75 & 2.3523 .75 \\
\hline$(5,1)$ & 1 & 9 & 7 & 10 \\
\hline 144 & $1.5 \% 1.4 \% 0.9 \%$ & $0.4 \% 0.3 \% 0.1 \%$ & $7.6 \% 5.2 \% 3.3 \%$ & $0.3 \% \quad 0.2 \% 0.3 \%$ \\
\hline $10^{5} \times$ & 0.050 .050 .25 & 0.653 .650 .75 & 0.50 .60 .65 & 86.67 .6 \\
\hline$(6,1)$ & 20 & 56 & 35 & 28 \\
\hline 624 & $36.1 \% 16.6 \% 8 \%$ & $4.4 \% 2.7 \% 0.5 \%$ & $22 \% 12.1 \% 4.8 \%$ & $28.7 \% 44.2 \% 5 \%$ \\
\hline $10^{5} \times$ & 7.557 .755 .8 & 30.833 .134 .2 & 14.614 .815 .5 & 17.217 .216 .6 \\
\hline$(7,1)$ & 38 & & & 53 \\
\hline 2256 & $12.9 \% 6.6 \% 2 \%$ & - & - & $5.2 \% 5 \% 0.6 \%$ \\
\hline $10^{5} \times$ & 16.318 .519 .4 & & & 33.23021 .9 \\
\hline$(4,2)$ & & 51 & & 43 \\
\hline 960 & - & $1.2 \% \quad 0.8 \% 0.5 \%$ & - & $3.1 \% 2.1 \% 5.8 \%$ \\
\hline $10^{5} \times$ & & 108.97 .7 & & 403225.6 \\
\hline$(5,2)$ & & & & 43 \\
\hline 4080 & - & - & - & $23.2 \% 14.5 \% 9.7 \%$ \\
\hline $10^{6} \times$ & & & & 14.112 .511 .4 \\
\hline$(6,2)$ & & & & 43 \\
\hline 17568 & - & - & - & $17.3 \% 11.8 \% 7 \%$ \\
\hline $10^{6} \times$ & & & & 15.314 .814 \\
\hline$(7,2)$ & & & & 43 \\
\hline 71376 & - & - & - & $2.9 \% \quad 0.5 \% 1.9 \%$ \\
\hline $10^{6} \times$ & & & & 22.72117 .1 \\
\hline Simulation gain $(\mathrm{dB})$ & 13.421 .427 .7 & 12.416 .321 & 12.717 .822 .1 & 1.78 .114 .5 \\
\hline
\end{tabular}

Table C.4: Error floor estimation of the WiMAX2 $(1152,960)$ LDPC code using $M-\mathrm{QAM}$ signalling. The number of estimated TSs, the relative contribution of a given TS in the total estimated FER, and the total number of trials for the estimation of a TS class are provided in each case. 